\title{
ELECTRICAL DISCHARGE MILLING WITH OBLONG TOOLS
}




\title{
ELECTRICAL DISCHARGE MILLING WITH OBLONG TOOLS
}

\author{
by \\ IVAN J. MIKESIC, B.ENG

\begin{abstract}
A Thesis
Submitted to the School of Graduate Studies

in Partial Fulfillment of the

Requirements for the Degree of
\end{abstract}

Master of Applied Science

McMaster University

Hamilton, Ontario, Canada, 2007

(c) Copyright by IVAN J. MIKESIC, November 2007 
Master of Applied Science

Mechanical Engineering
McMaster University

Hamilton, Ontario
TITLE:

AUTHOR:

SUPERVISORS:

NUMBER OF PAGES: $\quad$ xi, 113 


\section{Abstract}

Electrical discharge machining $(\mathrm{EDM})$ is perhaps the most prevalent nontraditional machining process in industry. Therefore, a great amount of effort has been invested towards improving the process. One result of these efforts was the introduction of CNC capabilities to EDM machine tools. Though CNC capabilities are predominantly used for performing orbital EDM, they may also be used for electrical discharge (ED) milling.

Cylindrical tools are typically used in ED milling. While other tooling concepts have been developed for use with CNC EDM, this area of EDM is lacking in research. In this work an oblong set of tools for ED milling is presented. The set contains tools capable of machining cavities with both round and sharp features. Motivation for development of the tool set is discussed along with the benefits accompanied by the use of these tools.

The cavity profiles considered in this work are divided into three groups based on concavity, continuity and geometric composition. Based on these groups, algorithms are presented for tool type selection and tool size calculation. Tool path planning strategies for each of the tool types are also presented.

Two examples are presented in which the algorithms are applied to 2D profiles. The first of these profiles is star shaped, while the second is gear shaped. Both examples were machined on a 4-axis CNC EDM using the oblong tools presented in this work. When compared to equivalent cylindrical/square tools, the oblong tools used for the machining of the cavities provided a substantial increase in frontal area corresponding to improved material removal rates. Furthermore, for both examples, the complete machining of a single layer is accomplished with only one pass of the tool. 


\section{Acknowledgements}

I would like to express my deepest gratitude to my supervisors, Dr. Robert Fleisig and Dr. Philip Koshy. Their encouragement and guidance have been outstanding. Their knowledge and advice have helped me develop both academically and as an individual. For this, I can't thank them enough.

I am grateful for the opportunity to study in the McMaster Manufacturing Research Institute. I would like to thank Warren Reynolds for his assistance with the AGIE IMPACT 2. I would also like to express my gratitude to Jim McLaren, Mark McKenzie, Ron Lodewyks and Dave Schick for their valuable advice and assistance in the machine shop.

Special thanks to Dr. Koshy's research group for their valuable advice, support and friendship. I would also like to thank Dr. Spence and his research group for their generosity and advice.

I would like to express appreciation to Martin Held for providing VRONI, a software application used to compute two-dimensional Voronoi diagrams and medial axis transforms.

I would like to express my gratitude to my family and friends. In particular, I would like to thank my mother, father, brother and grandparents for their love and support.

Last, but certainly not least, I would like to thank Elycia Watson for the encouragement and motivation she has provided me. Thank you for the countless hours spent discussing and reviewing this work. I am truly grateful for your support. 


\section{Contents}

1 Introduction $\quad 1$

1.1 Electrical Discharge Machining (EDM) . . . . . . . . . . . . 2

1.2 Die-Sinking EDM . . . . . . . . . . . . . . . . . . . . 3

1.3 Wire EDM . . . . . . . . . . . . . . . . . . . . . 6

1.4 Electrical Discharge Milling . . . . . . . . . . . . . . 7

1.5 Scope \& Organization of Present Work . . . . . . . . . . . . . . . . . 10

2 Literature Survey $\quad 11$

2.1 Tool Wear Compensation for ED Milling . . . . . . . . . . . . . . 12

2.1.1 On-line Tool Wear Compensation . . . . . . . . . . . . . . . 12

2.1.2 Off-line Tool Wear Compensation . . . . . . . . . . . . 16

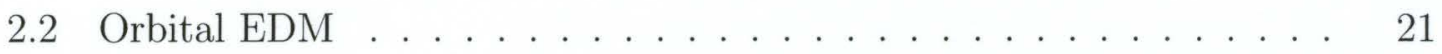

2.3 Innovative Tooling Concepts for CNC EDM . . . . . . . . . . . 25

3 Tool Set $\quad 32$

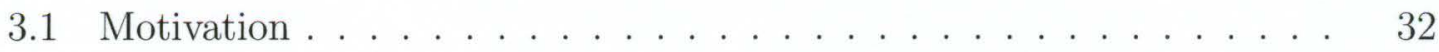

3.2 Development of a Tool Set . . . . . . . . . . . . . . . 34

4 Tool Type Selection and Tool Size Calculations 37

4.1 Profile Definitions . . . . . . . . . . . . . . . . 37 
4.2 Tool Type Selection . . . . . . . . . . . . . . . . . . . . . . . 41

4.3 Medial Axis Transform (MAT) . . . . . . . . . . . . . . . . . 44

4.4 Tool Width Calculation . . . . . . . . . . . . . . . . . 45

4.4.1 Tool Width Calculation - Profile Type $1 \ldots \ldots$

4.4.2 Tool Width Calculation - Profile Type $2 \ldots \ldots$. . . . . 47

4.4.3 Tool Width Calculation - Profile Type $3 \ldots \ldots$. . . . 50

4.4.4 Tool Width Calculation - General Algorithm . . . . . . . . 50

4.5 Tool Length Calculation . . . . . . . . . . . . . . . . . . . 52

4.5.1 Tool Length Calculation - Profile Type 1 . . . . . . . . . 58

4.5.2 Tool Length Calculation - Profile Type 2 . . . . . . . . 59

4.5.3 Tool Length Calculation - Profile Type $3 \ldots \ldots$

4.5.4 Tool Length Calculation - General Algorithm . . . . . . . . 61

5 Tool Path Planning $\quad 63$

5.1 Tool Path Planning - Profile Type $1 \ldots \ldots$. . . . . . . . 67

5.2 Tool Path Planning - Profile Type $2 \ldots \ldots \ldots$

5.3 Tool Path Planning - Profile Type $3 \ldots \ldots$. . . . . . . 73

5.4 Tool Path Planning - General Algorithm . . . . . . . . . . 76

6 Experiments \& Results $\quad 79$

6.1 Example 1 - Star Shaped Cavity . . . . . . . . . . . . . . . . 80

6.2 Example 2 - Gear Shaped Cavity . . . . . . . . . . . . . . . 94

$\begin{array}{lll}7 & \text { Conclusions and Recommendations } & 105\end{array}$

7.1 Summary . . . . . . . . . . . . . . . . . . . 105

7.2 Conclusions . . . . . . . . . . . . . . . . . . 107

7.3 Recommendations . . . . . . . . . . . . . . . . . . . . . 108 


\section{List of Figures}

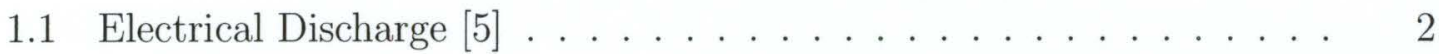

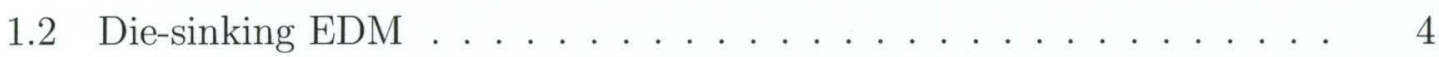

1.3 Orbital EDM (Circular Orbit) $[5] \ldots \ldots \ldots \ldots \ldots$

1.4 Example of a Component Machined by Die-Sinking EDM [7] . . . . 6

1.5 Wire EDM . . . . . . . . . . . . . . . . . . . 6

1.6 Example of a Component Machined by Wire EDM [7] . . . . . . . 7

1.7 ED Milling . . . . . . . . . . . . . . . . . . 8

1.8 ED Milling Example $[10] \ldots \ldots \ldots \ldots$

2.1 Simulation of Tool Wear Compensation Techniques [17] . . . . . . 14

2.2 On-line Tool Length Measurement $[22] \ldots \ldots \ldots$

2.3 UWM - Zig-Zag Tool Path $[20] \ldots \ldots \ldots \ldots \ldots$

2.4 Micro ED Milling Examples (Adapted from [23]) . . . . . . . . . 18

2.5 EDM Slot Machining [25] $\ldots \ldots \ldots \ldots$

2.6 EDM Slot Machining Results $[25] \ldots \ldots \ldots$

2.7 Cavity Machined using Linear Path Model [26] . . . . . . . . . . 20

2.8 Common Orbits: (a) Linear, (b) Circular, (c) Square, (d) Equimode . 21

2.9 Flushing by Orbital Motion $[28] \ldots \ldots \ldots \ldots \ldots$

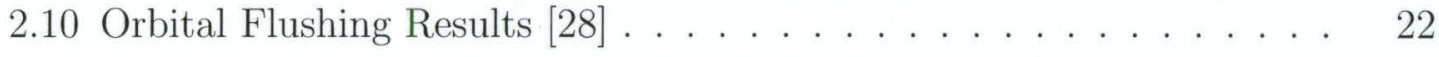


2.11 MRR vs. Pulse Interval at Different Machining Areas [29] . . . . . . 23

2.12 Tool Types used in CNC EDM [33] . . . . . . . . . . . . . . . . . . 26

2.13 CNC EDM using Frame Type Tools $[34] \ldots$. . . . . . . . . . . . . 27

2.14 MRR vs. Current with Constant Tool Size [9] . . . . . . . . . . . . . 29

2.15 Unmodified and Modified Reuleaux Triangles (Adapted from [35]) . . 30

2.16 Cavities Machined by a Rotating Reuleaux Triangle (Adapted from [35]) 31

3.1 MRR vs. Current Density $[36] \ldots \ldots$. . . . . . . . . . . . . . . . 33

3.2 Oblong Tool Set . . . . . . . . . . . . . . . . . . 35

4.1 (a) Concavity and (b) Tangent Continuity . . . . . . . . . . . . 38

4.2 Profile Type 1 Example . . . . . . . . . . . . . . . . . . . . . . . . . . 39

4.3 Profile Type 2 Example . . . . . . . . . . . . . . . . . . . . . . . . 40

4.4 Profile Type 3 Example . . . . . . . . . . . . . . . . . . . . . . . . . 40

4.5 Profile/Tool Type Pairs . . . . . . . . . . . . . . . . . . . 42

4.6 Tool Type Selection Algorithm . . . . . . . . . . . . . . . 43

4.7 Medial Axis Transform of a 2D Profile . . . . . . . . . . . . . . . 44

4.8 Tool Width Constraint Defined by Smallest Corner Radius . . . . . . 46

4.9 Cavity Profile with Lines Removed due to Condition 1 . . . . . . . . 48

4.10 Tapers - (a) Lines Removed and (b) Lines Remain . . . . . . . . . . . 49

4.11 Medial Axis and Modified Medial Axis . . . . . . . . . . . . . . 49

4.12 Tool Width Algorithm . . . . . . . . . . . . . . . . 51

4.13 Tool Length Defined by $\mathrm{MIC}-\mathrm{L}_{1}>\mathrm{L}_{3}>\mathrm{L}_{2} \ldots \ldots$. . . . . . . . . . 52

4.14 Tool Length Determined by MIC is not Maximal . . . . . . . . . . . 53

4.15 Centre Lines of Tool Type 1 and $2 \ldots$. . . . . . . . . . . . 54

4.16 Tool Centre Lines Engaged in Point and Line Contact . . . . . . . . . 54

4.17 Bounded Region - Interior Angle $\geq 90^{\circ} \ldots \ldots . \ldots . . \ldots 5$ 
4.18 Bounded Region - Interior Angle $<90^{\circ} \ldots \ldots$. . . . . . . . . . 56

4.19 Tool Length Defined by Convex Regions on the Profile . . . . . . . . 57

4.20 Variables in Eqn. $4.2 \ldots \ldots \ldots$. . . . . . . . . . . . . 59

4.21 Length - Round and Square End of Tool Type 3 . . . . . . . . . . . . 60

4.22 Tool Length Algorithm . . . . . . . . . . . . . . . . . . . . . . 61

5.1 Coordinate System for a 4-Axis EDM Machine Tool . . . . . . . . . . 64

5.2 (a) Single Pass Required and (b) Multiple Passes Required . . . . . . 66

5.3 Determination of Critical Points . . . . . . . . . . . . . . . 67

5.4 Tool Constrained to the Offset Curve . . . . . . . . . . . . 68

5.5 (a) Tool Normal and (b) Not Normal to Profile at Starting Point . . . 69

5.6 Tool Constrained to the Profile . . . . . . . . . . . . . 71

5.7 Profile Type 2 - Critical Points . . . . . . . . . . . . . . . . . 72

5.8 Trailing Point Tracing the End Portion of a Curve . . . . . . . . . . . 73

5.9 Profile Type 1 Representation of a Type 3 Profile . . . . . . . . . . . 74

5.10 Profile Curve Traced by Only the Round End of the Tool . . . . . . . 76

5.11 Tool Path Planning Algorithm . . . . . . . . . . . . . 77

6.1 Star Shaped Cavity (Dimensions in $\mathrm{mm}$ ) . . . . . . . . . . . . 80

6.2 Curves Composing the Profile of the Star Shaped Profile . . . . . . . 81

6.3 Star Shaped Cavity - Medial Axis . . . . . . . . . . . . . . 82

6.4 Star Shaped Cavity - MIC and Corresponding Tool Length . . . . . . 83

6.5 Star Shaped Cavity - Profile Offset by Half the Tool Width . . . . . 84

6.6 Star Shaped Cavity - Results of Tool Length Search . . . . . . . . . . 84

6.7 Star Shaped Cavity - Maximum Calculated Tool Size . . . . . . . . . 85

6.8 Star Shaped Cavity - Tool Size Comparison . . . . . . . . . . . 86

6.9 Star Shaped Cavity - Profile Offset by Tool Length . . . . . . . . . . 87 
6.10 Star Shaped Cavity - Tool Path Segments A \& B . . . . . . . . 88

6.11 Star Shaped Cavity - Tool Path Segments C \& D . . . . . . . . . 89

6.12 Star Shaped Cavity (Maximum Tool Size) - Tool Path Segments A \& B 90

6.13 Star Shaped Cavity (Maximum Tool Size) - Tool Path Segments C \& D 91

6.14 Tool used in ED Milling of the Star Shaped Cavity . . . . . . . . . . 92

6.15 Experimental Setup . . . . . . . . . . . . . . . . 93

6.16 Star Shaped Cavity Machined by ED Milling with Tool Type 1 . . . . 93

6.17 Gear Shaped Cavity (Dimensions in $\mathrm{mm}$ ) . . . . . . . . . . . . . . 94

6.18 Curves Composing the Profile of the Gear Shaped Cavity . . . . . . . 95

6.19 Gear Shaped Cavity - Features of Minimum Length and Radius . . . 96

6.20 Gear Shaped Cavity - MIC and Corresponding Tool Length . . . . . 97

6.21 Gear Shaped Cavity - Convex Region and Resulting Tool Length . . 98

6.22 Gear Shaped Cavity - Tool Length Results for Convex Island . . . . 98

6.23 Gear Shaped Cavity - Tool Size Comparison . . . . . . . . . . . . . . 99

6.24 Gear Shaped Cavity - Tool Path Segments C \& D . . . . . . . . . . 100

6.25 Gear Shaped Cavity - Tool Path Segments C \& D . . . . . . . . . . . 101

6.26 Tool used in ED Milling of the Gear Shaped Cavity . . . . . . . . . . 102

6.27 Gear Shaped Cavity Machined by ED Milling with Tool Type 2 . . . 103 


\section{Chapter 1}

\section{Introduction}

CAD/CAM systems aid engineers and designers in the cost effective production of quality products. The life cycle of these products is divided into two main categories; the design process and the manufacturing process. In the design process, CAD systems are used to create, modify, analyze, and optimize the product. During the manufacturing process, CAM systems are used to plan, manage, and control the processes required to manufacture the product [1].

One of the most important and developed areas of CAD/CAM systems is computer numerical control (CNC). This is the use of programmed instructions for the control of a machine tool [1]. Today, these instructions are standardized, most commonly as G-code.

CNC technology has had a tremendous effect on the manufacturing industry in terms of flexibility. Approximately $15 \%$ of machine tools in the U.S. are CNC machines. Furthermore, these machines account for $60 \%$ of production [2]. Due to this success, CNC capabilities have been added to many types of machine tools including those used for electrical discharge machining. 


\subsection{Electrical Discharge Machining (EDM)}

Electrical discharge machining (EDM) is the process of removing material from a workpiece by means of rapid and repetitive electrical discharges [3]. Discharges occur in a gap between the tool and the workpiece, making EDM a non-contact process. The gap is filled with a dielectric fluid and typically has a width of $10-100 \mu \mathrm{m}$ [4].

For a discharge to initiate, the dielectric medium must be broken down (Fig. 1.1a). This is achieved by applying a pulsed voltage sufficient to overcome the dielectric breakdown strength of the gap. Once the dielectric is broken down, current flows across the gap resulting in the formation of a plasma channel (Fig. 1.1b). High temperatures in the plasma channel cause melting and evaporation of the tool and the workpiece. The current is then switched off and the dielectric recovers its strength (Fig 1.1c). This is accompanied by a rapid drop in temperature and pressure which causes an implosion of the plasma channel. The molten debris is evacuated from the workpiece by this implosion and flushed away from the gap by the dielectric fluid [4].
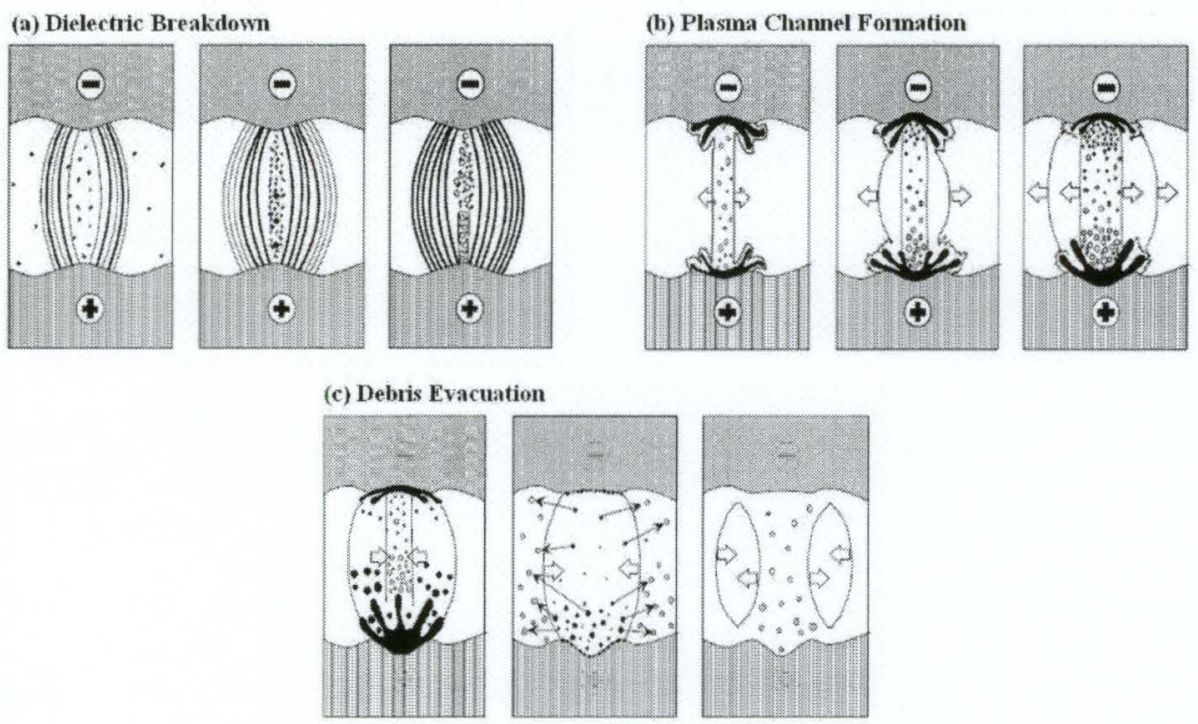

Figure 1.1: Electrical Discharge [5] 
The material removal mechanism in EDM gives it advantages that are not common to other material removal processes. One of these advantages is the ability to machine any conductive material regardless of hardness. This can be done with EDM because material removal is a thermal process as opposed to a mechanical process. Machining of hard materials using a mechanical process, such as milling, would cause the tool to experience high forces, vibrations, and possibly breakage. Furthermore, as the tool size decreases in mechanical processes, the chance of failure due to vibration and cutting forces increases. Due to its non-contact nature, the cutting forces are negligible which implies that the size of the tool and thus the machined features may be extremely small. This makes EDM a good candidate for micro-machining. Another advantage of EDM is the ability to machine internal sharp corners. Since sparks occur across the entire gap, the shape of the tool is imprinted on the workpiece. This means that if the tool is manufactured to have a sharp corner, that corner can be machined on the workpiece.

There are three types of EDM commonly used: die-sinking EDM, wire EDM, and ED milling. The first of these processes to become commercially available was die-sinking EDM.

\subsection{Die-Sinking EDM}

In die-sinking EDM, the tool is manufactured as a shaped which is complimentary to the desired cavity. This allows for the tool to imprint its shape into the workpiece as it advances towards it. Fig. 1.2 shows this process. It shows the direction of motion of the tool and the tool shape imprinted into the workpiece. 


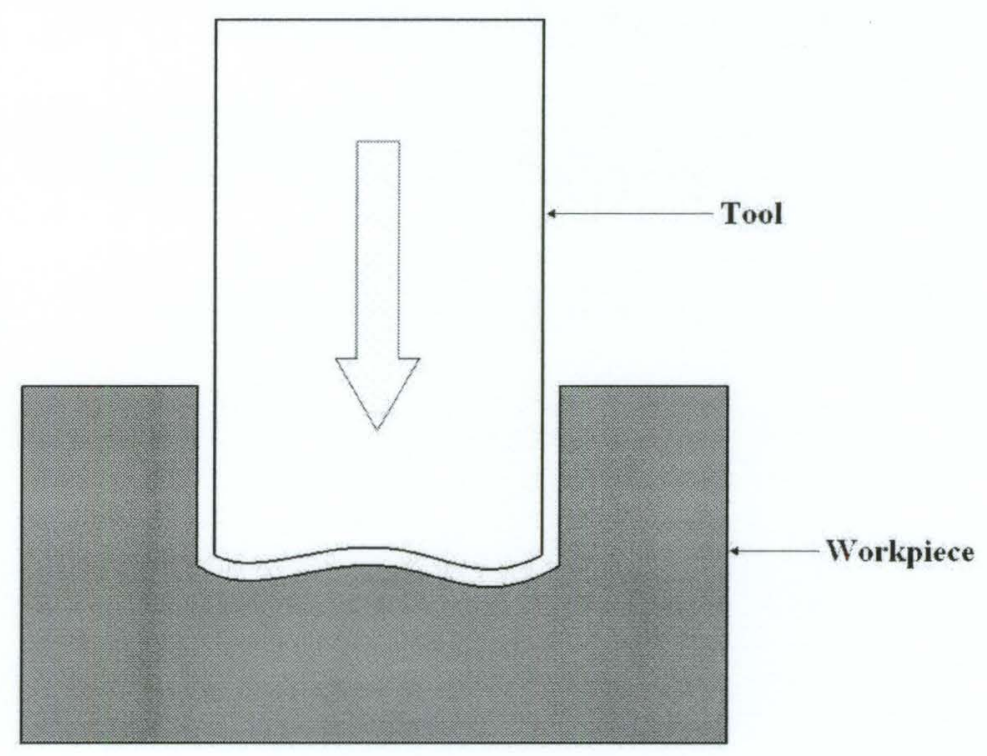

Figure 1.2: Die-sinking EDM

The tools used in die-sinking EDM are typically made of copper or graphite and are manufactured on CNC milling machines. Depending on the specified tolerances, several electrodes may be required for roughing, semi-finishing, and finishing operations. As a result, the tooling costs for this type of EDM are high and generally account for $50 \%$ of manufacturing costs [6].

Flushing is important in EDM as it promotes uniform distribution of debris in the gap. Under poor flushing conditions, debris collects between the tool and the workpiece causing localized discharges and thus, arcing. In die-sinking EDM, flushing is difficult because the tool essentially covers the entire cavity. This results in very little room for debris to evacuate the gap and results in a poor surface due to arcing. Some strategies have been developed to improve the removal of debris from the gap. One of these strategies is jump EDM in which the tool is periodically retracted from the workpiece allowing for debris from the gap to be removed. However, when the tool is retracted from the workpiece, it is not machining and hence compromises productivity. 
Another strategy which has been developed to improve flushing during diesinking is orbital EDM. In this type of EDM the tool resembles the cavity geometry, but is smaller. In order for the tool to machine the cavity, there must be relative motion between the tool and workpiece, along an orbit. There are many different types of standard orbits, such as circular, star, and equimode orbits. Fig. 1.3 shows a workpiece whose orbit is circular.

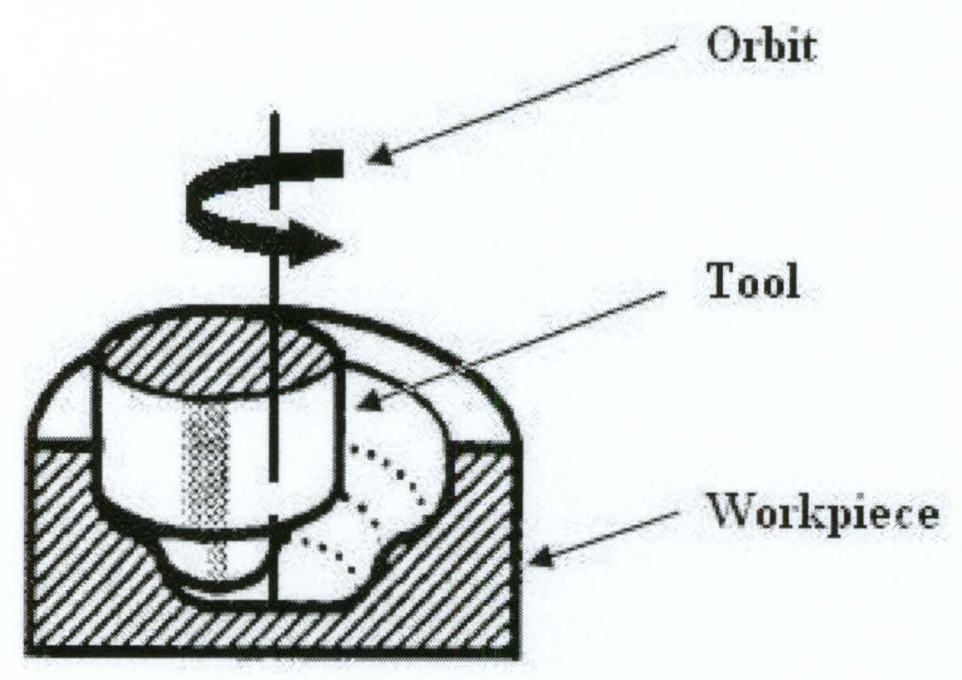

Figure 1.3: Orbital EDM (Circular Orbit) [5]

Die-sinking EDM is most commonly used in the tool and die industry. It offers many advantages over mechanical milling processes including the ability to machine internal sharp corners and deep cavities. Furthermore, EDM tools can be designed to reach places that are inaccessible to milling cutters. This is especially important in the machining of cooling holes for dies. Fig. 1.4 shows a sample die made using die-sinking EDM. 


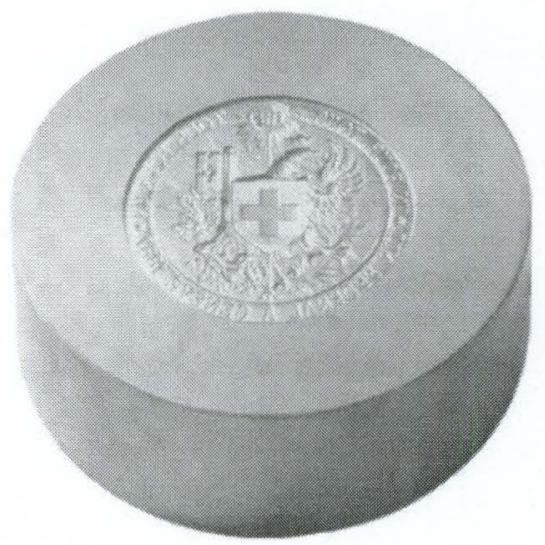

Figure 1.4: Example of a Component Machined by Die-Sinking EDM [7]

\subsection{Wire EDM}

In wire EDM, the electrode is in the form of a wire which moves along its axis to perform through cut as it is traversed across the workpiece along a programmed path. Some common wire materials include brass and molybdenum typically $20-300 \mu \mathrm{m}$ in diameter. This process is shown in Fig. 1.5.

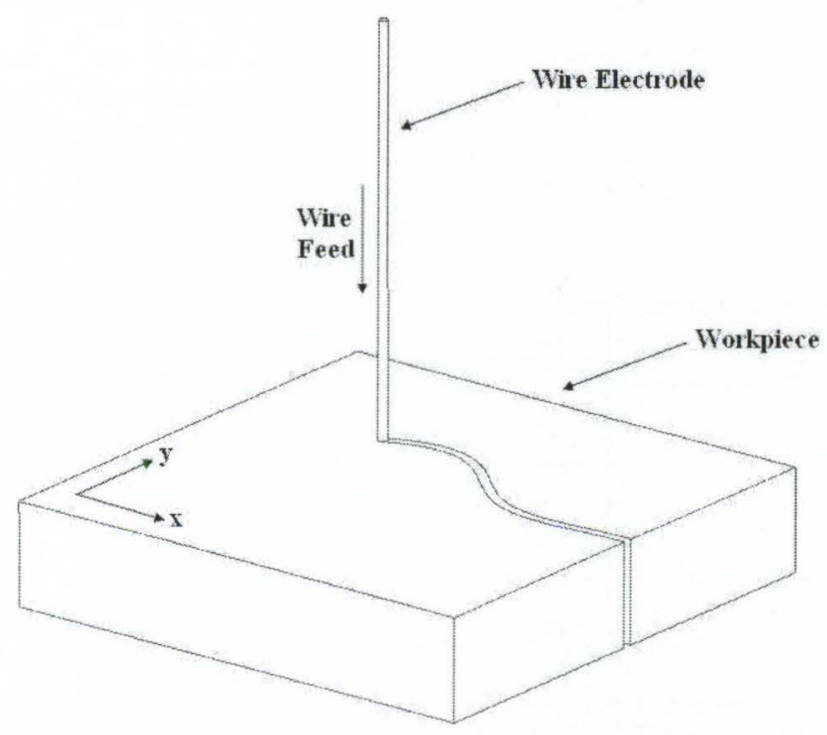

Figure 1.5: Wire EDM 
In wire EDM, the wire must span the entire workpiece thickness. This requires a pre-formed hole in the workpiece through which the wire may be fed. Alternatively, the wire may begin machining from an edge of the workpiece.

In wire EDM, there are two wire guides, located above and below the workpiece. Independent control of these guides allows for the machining of tapers. This is achieved through the controlled misalignment of the wire guides, so as to tilt the wire and thus machine tapers.

Wire EDM is the most commonly used type of EDM [8]. It has many applications including the manufacture of extrusion dies. Fig. 1.6 shows an extrusion die machined by wire EDM.

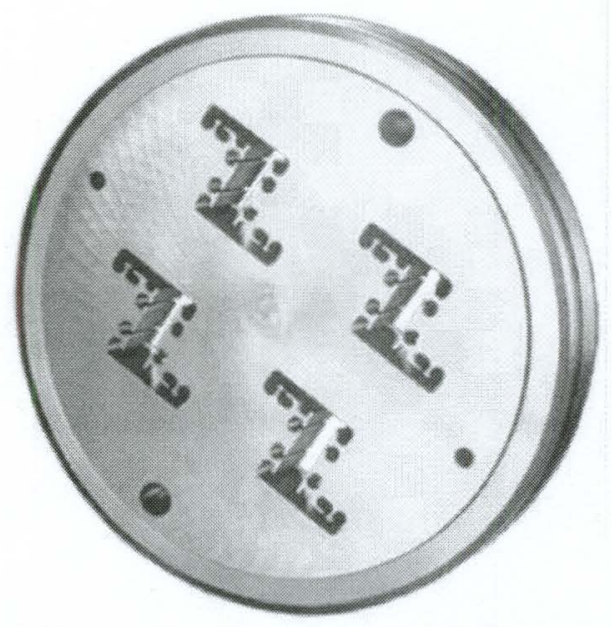

Figure 1.6: Example of a Component Machined by Wire EDM [7]

\section{$1.4 \quad$ Electrical Discharge Milling}

During ED milling, a simple shaped solid or hollow electrode is traversed along a programmed path. In this process, a layer-by-layer approach is generally used in tool path generation. In each layer, the tool path must be designed such that the 
frontal area of the tool intersects the area bounded by the cavity profile. This may be accomplished with an offset approach or a zig-zag strategy. Fig. 1.7 shows the ED milling of a cavity using the offset approach.

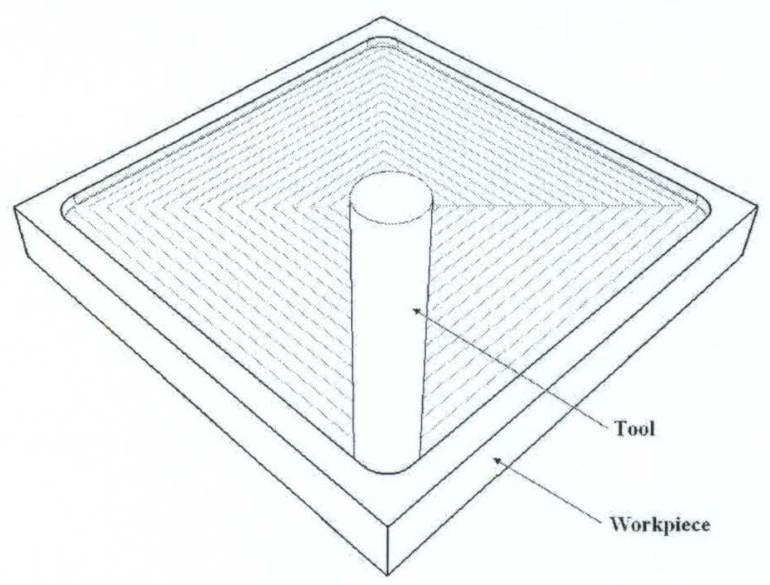

Figure 1.7: ED Milling

Tools used in ED milling are most commonly made from copper or graphite. The simple shape and size of the tools make this process much more cost effective than die-sinking EDM in terms of tool manufacturing costs. Furthermore, the tool manufacture time can be minimized or eliminated by using standardized bar stock.

Another benefit of using ED milling as opposed to die-sinking EDM is flushing effectiveness. This advantage is a result of the difference in frontal area between the tool and the machined cavity. In ED milling, the tool is much smaller than the cavity providing ample space for the dielectric fluid to penetrate the gap. The result is a sufficiently flushed gap and nearly $100 \%$ effective discharges [9].

Though the size of the tool used in ED milling provides desirable flushing conditions, it limits the amount of electrical power which may be passed through the tool. This results in lower material removal rate (MRR) and therefore increased machining times. This aspect represents a major motivation for the work presented in this thesis. 
One of the issues in ED milling which has received much attention is tool wear. During EDM, material is removed from both the tool and the workpiece. This causes the tool to experience a change in shape and length. Research efforts have been made to compensate for these changes using both on-line and off-line methods. The most popular methods in practice are off-line compensation of tool wear and incremental redressing of the tool.

Until recently, ED milling did not have many industrial applications. It was with the increasing demand for micro-parts that this has changed. Since the forces in EDM are negligible, it is a preferred process for micro-machining. More specifically, ED milling is a perfect candidate for micro-machining. Die-sinking EDM is less suitable because it requires the manufacture of a micro-tool.

As the tool size in ED milling approaches the micro-scale, tool wear becomes more significant. Furthermore, jet flushing may cause the tool to experience vibrations and thus result in unsatisfactory tolerances. However, these issues have been and are continually being addressed to improve the process. Fig. 1.8 shows some micro-parts machined by ED milling. Further applications of ED milling include the machining of ceramics, features in turbine blades and medical devices.

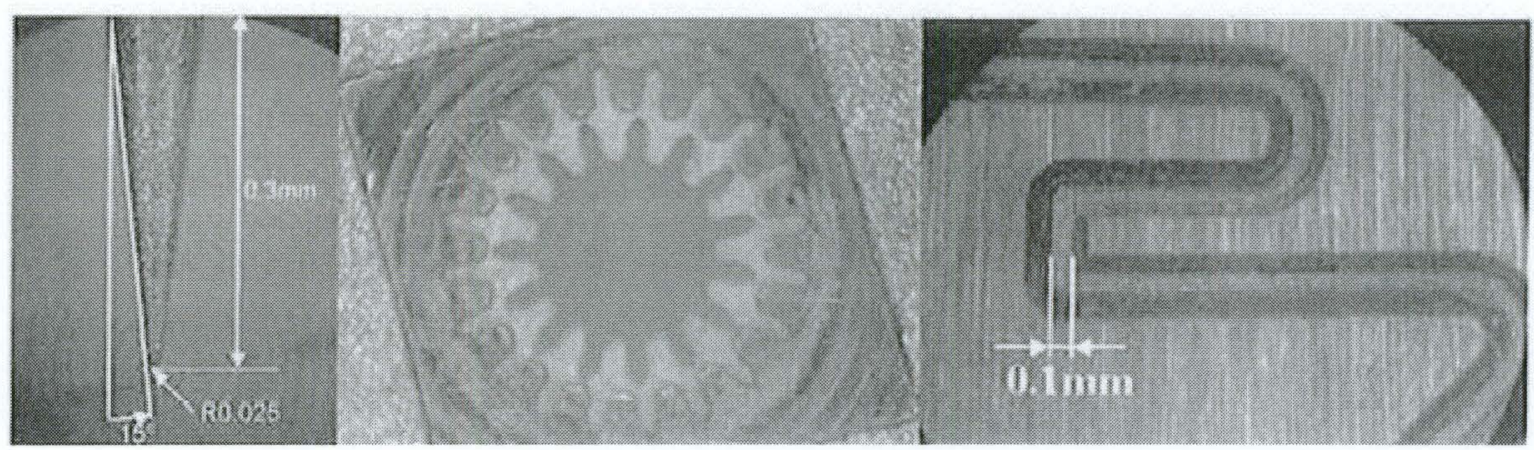

Figure 1.8: ED Milling Example [10] 


\subsection{Scope \& Organization of Present Work}

The focus of this work is to propose a new approach for ED milling of 2D blind cavities. Development of an oblong tool set, which differs from the cylindrical tools typically used in ED milling, forms the basis of this approach. The motivation for the development of a new tool set comes from previous work in EDM which shows that there exists an optimum current density where maximum MRR can be achieved while maintaining a stable process.

Current research in ED milling is focussed on the use of cylindrical and ballnosed tools with emphasis on wear compensation through tool path planning and redressing of the tool. Little work has been done in exploring the possibility of different tool geometries.

In this work, a new tool set is presented along with algorithms for tool type selection, tool size constraints and tool path planning. Cavities considered in this work may be represented by $2 \mathrm{D}$ profiles, which have been divided into three groups based on concavity, continuity and geometric composition.

Each of the algorithms varies with the profile type and therefore are presented accordingly. At the end of each section, a general algorithm is presented which determines the profile type and an appropriate methodology. It is these algorithms which are used in implementation.

Worked examples of the algorithms are provided. Tool type selection, tool size constraints, and the tool path are computed for each cavity. Furthermore, two cavities that were machined on a 4-axis EDM are presented in this thesis. 


\section{Chapter 2}

\section{Literature Survey}

The addition of CNC capabilities to die-sinking EDM has lead to the development of orbital EDM and ED milling. Since their introduction, these processes have shown many advantages over die-sinking. Some of these advantages are improved flushing and reduced tooling costs.

Although there are many advantages to using orbital EDM and ED milling, these processes also have some drawbacks. The main concern is tool wear, more so in ED milling. As material is removed from both the tool and the workpiece, the tool changes its form and dimensions. Therefore, a significant research has been focussed on wear compensation strategies. Previously developed strategies have used on-line and off-line techniques.

One of the areas in ED milling lacking development is tooling. Most of the research conducted in ED milling deals with the use of rotating solid and tubular cylindrical tools. In order to fully exploit the advantages of this process, different tool geometries must be explored as cylindrical tools limit power capacity and thus MRR. Furthermore, cylindrical tools are not capable of machining sharp corners which is one of the benefits of the EDM process. 
In this chapter, a review of previous research conducted in CNC EDM and ED milling is presented. The review covers tool wear compensation strategies for ED milling, orbital EDM and innovative tooling concepts for CNC EDM.

\subsection{Tool Wear Compensation for ED Milling}

Tool wear is one of the main areas of interest in EDM as it directly affects the accuracy of machined surfaces. Many theories on electrode wear have been presented for die-sinking EDM $[11,12,13,14]$. However, these theories are not directly applicable to ED milling due to the relative motion between the tool and workpiece. Therefore, wear compensation strategies specific to ED milling have been developed and are reviewed in the following. Both on-line and off-line methods have been used to correct the tool path and compensate for wear.

\subsubsection{On-line Tool Wear Compensation}

Kaneko and Tsuchiya presented a technique for on-line tool wear compensation in 1988 [15]. Their work was based on periodically measuring the tool and correcting the path accordingly. A datum point on the top surface of the workpiece and a touch probe were used to measure the wear of the tool. The datum point on the workpiece was used to determine the change in length of the tool between successive measurements. The touch probe, made of cemented carbide, was used to measure changes in the radius of the tool. Upon measurement of the wear, the tool path was modified appropriately.

During the roughing process, layer-by-layer machining was performed with length compensation only. For further machining, radial compensation was considered and the frequency of the measurement/compensation cycle steadily increased to 
achieve the specified tolerances. The implementation of this system resulted in an undercut of $20 \mu \mathrm{m}$ which the authors attribute to improper specification of spark gap compensation.

In 2000, Charmilles Technologies patented a device and technique for measuring the length of an electrode [16]. By knowing the location of the measuring device and the position of the tool holder, the tool length can be calculated. The tool is lowered onto the measuring device and with a small amount of force from the tool (to overcome an opposing spring force), the device pivots causing a short-circuit between the measuring device and the contact electrode. When the short-circuit occurs, the tool length is measured allowing for tool path corrections to be made.

Another on-line compensation strategy for ED milling using hollow cylindrical tools was presented by Bleys et al. $[17,18]$. This work was based on pulse analysis and the efficiency factor [19]. The efficiency factor is defined as the ratio of total effective discharge time to total measurement time. It may be measured on-line allowing tool wear compensation to be performed in real-time. The following equation shows the relationship between volumetric wear and efficiency factor $[17,18]$.

$$
\Delta V o l_{E}=-K_{E} \cdot \int_{0}^{\Delta t} \Phi(t) d t
$$

In equation $2.1, \Phi(t)$ is the efficiency factor at time $t$ and $K_{E}$ is a constant dependent on machining conditions. To determine the change in length and thus the appropriate compensation in the z-direction, the volumetric wear $\left(\Delta \operatorname{Vol}_{E}\right)$ is divided by the cross-sectional area of the tool.

Simulated results for this technique showed that small errors $( \pm 10 \%)$ in the determination of $K_{E}$ resulted in increasingly large errors in tool path compensation. These errors are a result of continuous over compensation. Since $K_{E}$ is determined 
experimentally, an alternate technique was proposed, which Bleys et al. $[17,18]$ termed combined wear compensation.

Combined wear compensation is dependent on real-time pulse analysis and the off-line tool wear compensation strategy introduced by Yu. et. al. [20]. However, the off-line compensation strategy in this work uses a general representation of the blank surface and does not require a CAD model.

The novelty in this technique is the ability to machine workpieces with unexpected voids not shown in CAD models. This is due to the real-time pulse analysis. In the absence of voids, the smallest compensation value returned from the on-line and off-line techniques is used. Simulations of machining to a depth of $1 \mathrm{~mm}$ for each compensation technique proposed in [17] are shown in Fig. 2.1.

(a) Off-line Compensation

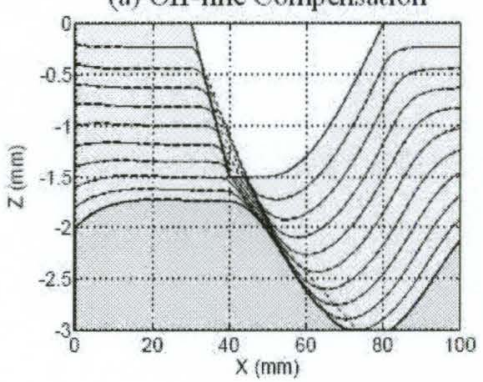

(b) On-line Compensation

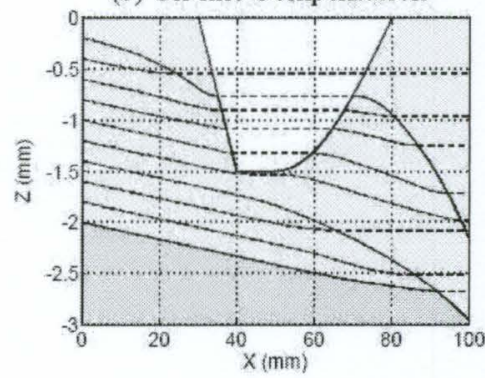

(c) Combined Compensation

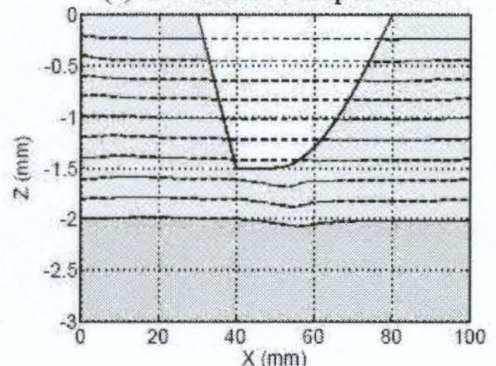

Figure 2.1: Simulation of Tool Wear Compensation Techniques [17]

The workpiece geometry used in these simulations contains a void. When off-line prediction was simulated, without knowledge of the void, the tool continued to advance across the void. The on-line pulse analysis simulation, with a $10 \%$ error in the estimation of $K_{E}$, results in over compensation during machining causing the tool to advance tool quickly in the z-direction. Simulation of the combined wear compensation, with a $10 \%$ error in the estimation of $K_{E}$, resulted in much smaller deviations. 
Applications of this technique include the machining of cast parts with unexpected voids and the repair of molds and dies. It has also been used in 4-axis tool path generation for rough machining by EDM using a ball-nosed tool [21].

Zhao et al. developed a CAD/CAM system for the micro-ED milling of 3D freeform cavities [22]. In this work, a slicing technique is proposed which intersects vertical lines with the surface boundary in any given layer. The intersections for a given layer are stored as a point list and are connected accordingly to generate the tool path for that layer. For even distribution of wear, the direction of the tool path alternates after each layer and rotates $90^{\circ}$ after every two layers.

In the machining of freeform surfaces, Zhao et. al. [22] suggest that off-line wear compensation strategies are not sufficient due to the complex mathematical representations of the surfaces. Therefore, a conductive standard block of known dimensions and accuracy is used to measure the change in electrode wear.

After the machining of each layer, the change in tool length is determined by contacting the block several times and taking the average of the resulting length measurements. Tool wear compensation for the next layer is based on this measurement. Furthermore, if the overall change in tool length is too large, the tool length is automatically increased using on-machine dressing. Fig. 2.2 shows the concept of the apparatus used to measure the change in tool length.

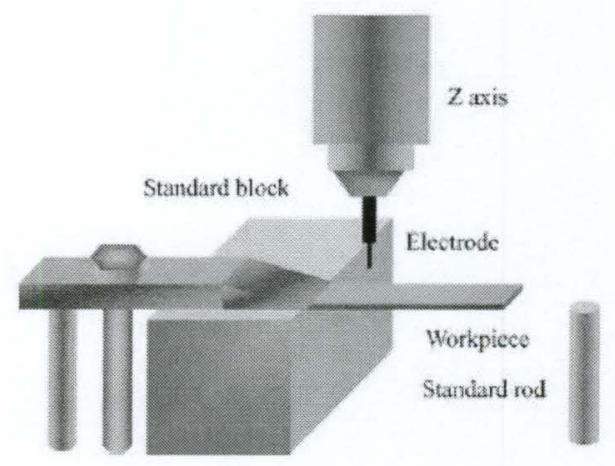

Figure 2.2: On-line Tool Length Measurement [22] 


\subsubsection{Off-line Tool Wear Compensation}

Off-line tool wear compensation is the most commonly used technique in ED milling. One of the major developments in this area is the uniform wear method (UWM) proposed by Yu et al. [20]. This method was designed for use with simply shaped tools and layer-by-layer machining strategies. It is proposed that under certain conditions, the shape of the electrode tip can be recovered after machining one layer.

In order to achieve this, an alternating zig-zag machining strategy was adopted. During the machining of a layer, the tool experiences wear causing inclination along the tool path. Therefore, when the tool advances to the next layer, it traverses the same path in the opposite direction. In order to further reduce this effect, the process is then carried out perpendicular to the original direction. This results in a more uniform surface on the bottom of the cavity and promotes uniform wear of the tool tip. When the tool geometry does not facilitate rotation, the use of this strategy is required to achieve such controlled wear. Fig. 2.3 shows this approach.

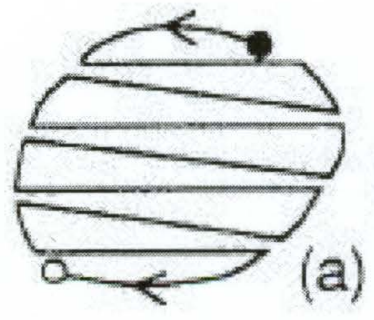

(a)

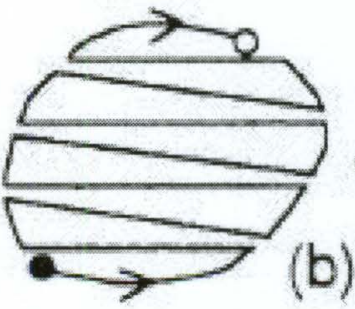

(b)
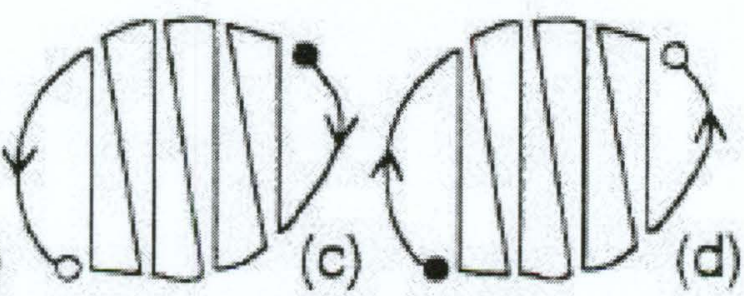

Figure 2.3: UWM - Zig-Zag Tool Path [20]

In addition to path planning in the xy-plane, feed per layer in the z-direction is calculated. The feed per layer is determined by an equation representing the total electrode wear. When rearranged, the equation may be used to determine the average machined depth for a given layer. This form of the equation is presented below [20]. 


$$
L_{w}=\frac{\Delta Z}{\left(1+v \cdot \frac{S_{w}}{S_{e}}\right)}
$$

In the above equation, $L_{w}$ is the average machined depth for a given layer, $\Delta Z$ is the feed per layer, $v$ is the volumetric wear ratio ${ }^{1}$, and $S_{w}$ and $S_{e}$ are the cross-sectional areas of the machined layer and the tool, respectively.

One of the criteria for uniform tool wear is that the condition $S_{e}<v \cdot S_{w}$ must be satisfied [20]. Since this value is constant, $\Delta Z$ may be used to control the average machined depth per layer. Therefore, the feed per layer is directly related to the required tolerance, resulting in a trade-off between accuracy and productivity.

The application of the UWM in CAD/CAM systems was researched by Rajurkar and $\mathrm{Yu}$ [23]. In this system, a CAD model of the part is sliced in the z-direction according to the feed per layer. The resulting cross-sectional areas for each layer are then calculated and passed to the CAM system. This information is used to modify the tool path generated by the CAM system such that it compensates for tool wear according to the UWM. The compensated cutter location (CL) file is then converted to $\mathrm{NC}$ codes by a post-processor.

This work was focussed on the machining of 3D micro-cavities. Therefore, cylindrical tools used in experiments were manufactured on the machine by wire electrical discharge grinding (WEDG) and had a diameter of $52 \mu \mathrm{m}$. The volumetric wear ratio was determined experimentally by slot machining. Fig. 2.4 shows some examples of the cavities machined using the CAD/CAM system developed in this work.

\footnotetext{
${ }^{1}$ The volumetric wear ratio is defined as the ratio of tool volume removed to workpiece volume removed
} 

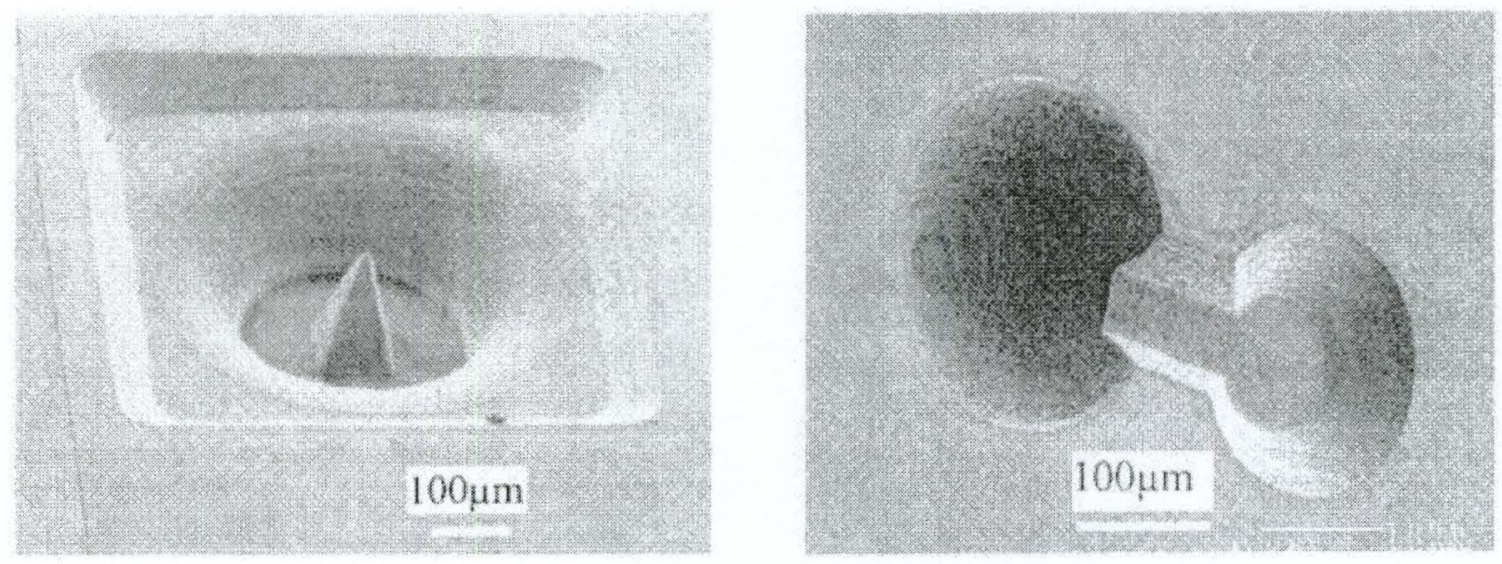

Figure 2.4: Micro ED Milling Examples (Adapted from [23])

Another approach to applying the UWM to CAD/CAM systems was made by Dimov et al. in 2003 [24]. In this work, an adaptive slicing algorithm was presented. Adaptive slicing determines the average thickness of each layer based on tolerances. Rearranging equation 2.2 to solve $\Delta Z$ for a given $L_{w}$ (average layer thickness), the feed per layer can be calculated independently for each layer. In the machining of a $1 \mathrm{~mm}$ diameter hemisphere, the number of slices produced using adaptive and uniform slicing were 18 and 71, respectively. The maximum deviation from the contour in both cases was $3 \mu \mathrm{m}$.

The machining of curved surfaces by ED milling is difficult due to tool wear. Yu et. al. approached this problem mathematically [25]. They suggested that given a mathematical representation of the workpiece surface and the desired contour, a tool path may be designed. The motivation for this work came from the tool wear characteristics during slot machining. If the tool is traversed along the slot without wear compensation, the surface produced differs from the path. However, the surface does depend on the tool path along with the initial surface of the workpiece and the wear characteristics of the electrode. This concept is illustrated in Fig. 2.5. 


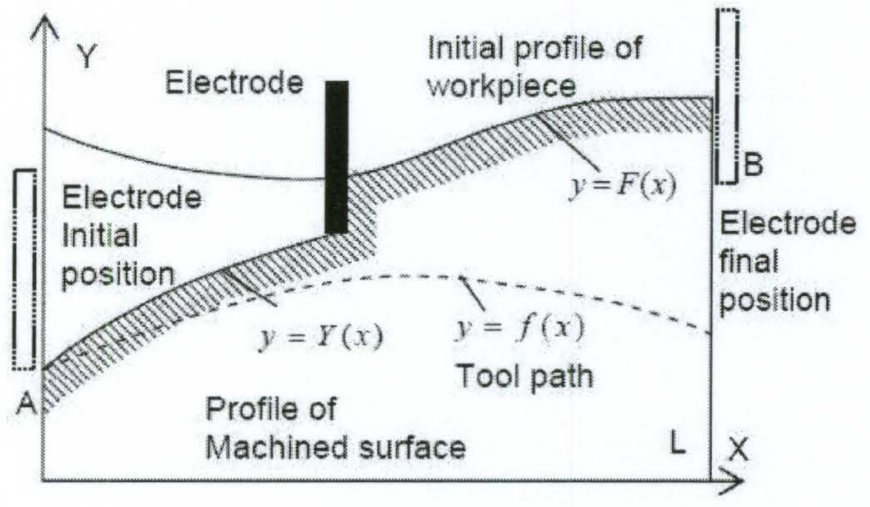

Figure 2.5: EDM Slot Machining [25]

Fig. 2.5 shows the initial workpiece profile, the tool path, and the workpiece profile after machining. The wear was represented as a change in tool length and was determined by the volumetric wear ratio. However, in order to use the volumetric wear ratio in this context, a wear factor, $C$, was developed. It is defined as $C=v \cdot \frac{w}{S_{e}}$, where $v$ is the volumetric wear ratio, $w$ is the tool width/diameter, and $S_{e}$ is the cross-sectional area of the tool. The wear factor, the initial workpiece surface and the tool path are related [25] to the desired surface by equation 2.3 .

$$
Y(x)=\exp (-C x) \cdot\left\{f(0)+\int_{0}^{x} \exp (-C x) \cdot\left[C F(x)+f^{\prime}(x)\right] d x\right\}
$$

$Y(x)$ is the profile of the machined surface, $F(x)$ is the machined profile, $f(x)$ is the tool path, $x$ is the horizontal distance from the starting point of the slot and $C$ is the wear factor.

Due to the complexity of the model, a simplifying assumption was required. The assumption was that the relationship between tool wear and tool position, $x$, is linear. This essentially means that curved surface profiles would be represented by several line segments. Computationally, this allows for the use of numerical methods and thus a simplified algorithm. Results comparing the simplified simulation to the machined surface are presented in Fig. 2.6 for both micro and macro ED milling. 

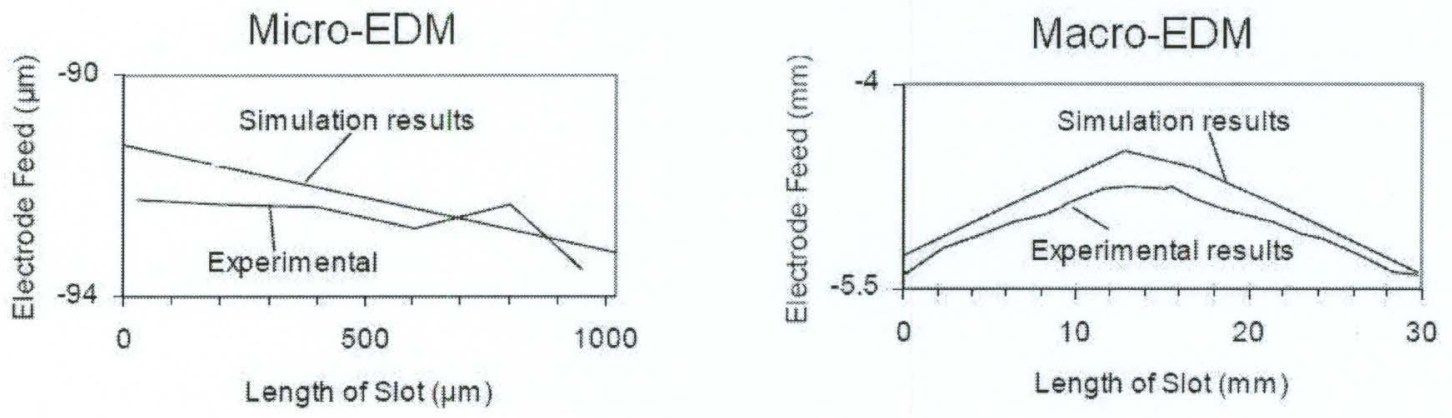

Figure 2.6: EDM Slot Machining Results [25]

The micro-profile was measured by contacting an electrode, of smaller diameter than the tool, with the machined surface. A coordinate measuring machine (CMM) was used to measure the macro-profile. It was suggested that differences in the simulation and experimental results for the micro-profile were partly due to the measurement technique and the presence of machining debris in the gap.

The use of this model for ED milling was further investigated by Narasimhan et al. [26]. Based on the mathematical wear compensation model developed by Yu et al. [25], mathematical representations of tool paths for linear and non-linear profiles were developed. A cavity was machined for which the tool path consisted entirely of linear segments. Applying the mathematically derived linear tool paths to the cavity shown in Fig. 2.7 resulted in a flatness error of $59 \mu \mathrm{m}$. Each step in the cavity elevation was $0.3 \mathrm{~mm}$.

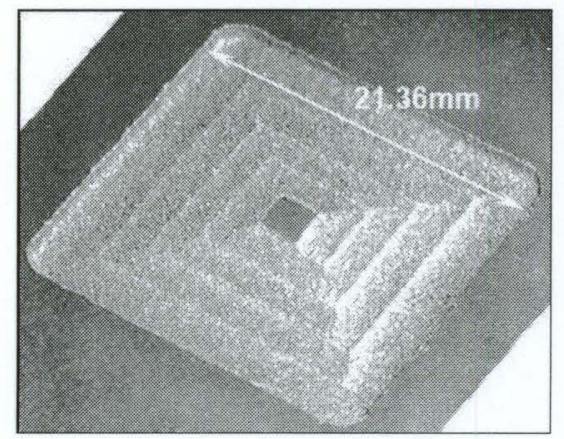

Figure 2.7: Cavity Machined using Linear Path Model [26] 


\subsection{Orbital EDM}

One of the early developments of CNC die-sinking EDM was orbital EDM. The introduction of this technique has led to many process developments relating to tool wear, flushing, discharge efficiency and accuracy $[8,27]$. These improvements are a result of relative motion between the tool and the workpiece. Some common orbits are shown in Fig. 2.8.

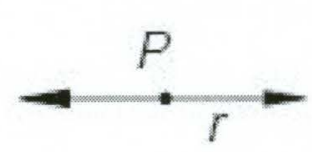

(a)

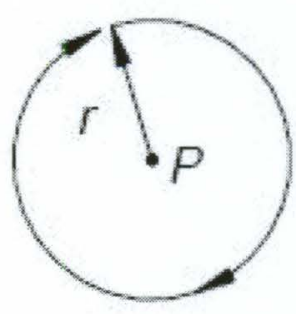

(b)

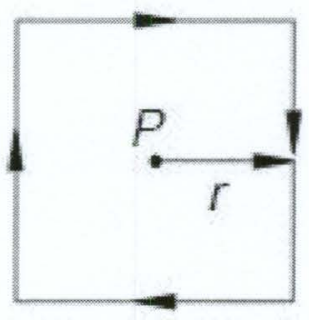

(c)

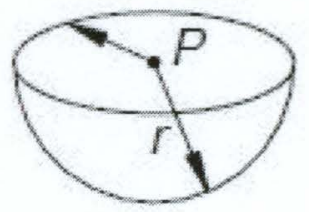

(d)

Figure 2.8: Common Orbits: (a) Linear, (b) Circular, (c) Square, (d) Equimode

Flushing in orbital EDM was studied by Masuzawa [28]. In this work, a selfflushing technique was proposed in which the tool orbits about the $\mathrm{x}$ or $\mathrm{y}$ axis depending on the orientation of the workpiece. Fig. 2.9 shows the relative motion between the tool and the workpiece used for this type of self-flushing.

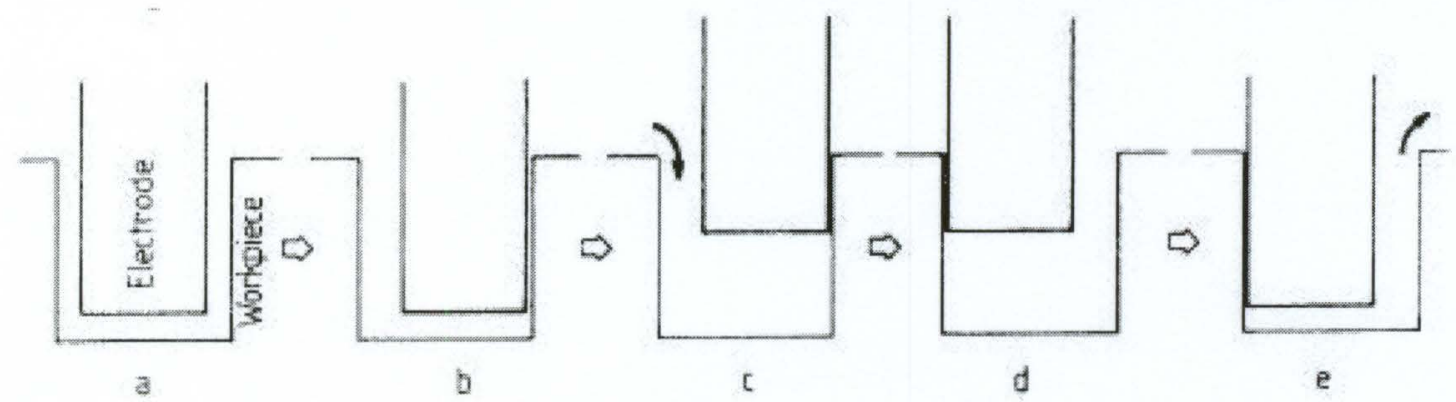

Figure 2.9: Flushing by Orbital Motion [28] 
Tests relating the machining time to the machined depth were used to characterize the performance of this technique. Also tested were die-sinking EDM without flushing and die-sinking EDM with jump flushing. It was found that the orbital flushing technique had shorter machining times and was free of arcing. Arcing occurred for both die-sinking EDM with and without jump flushing. Fig. 2.10 shows the results of these tests. The variables $x$ and $z$ refer to the motion along the $\mathrm{x}$ and $\mathrm{z}$ axes, respectively. Therefore, $x=0$ and $z=0$ refers to die-sinking, $x=0$ refers to jump flushing and non-zero values for both $x$ and $z$ refers to orbital machining. The dots in Fig. 2.10 indicate the incidence of severe arcing, at which point machining was stopped.

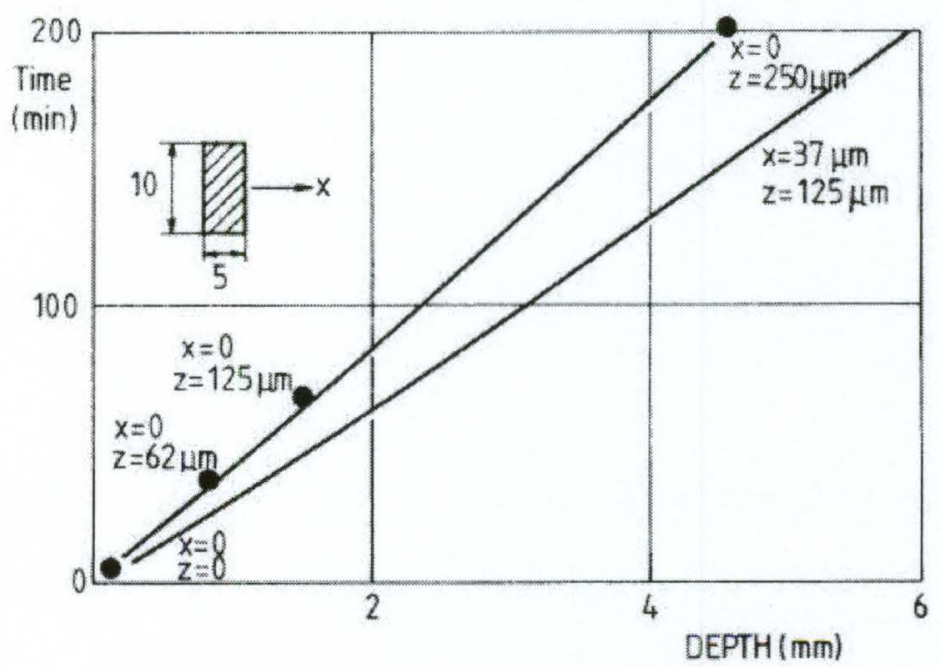

Figure 2.10: Orbital Flushing Results [28]

A study was performed by König et al. [29] which investigated the potential of orbital EDM for ED polishing. It was suggested that the kinematics of orbital EDM promote uniform distribution of debris in the gap resulting in improved process stability which is desirable for ED polishing. Furthermore, the planetary motion between the tool and the workpiece produces a leveling effect which removes any high points on the bottom surface of the workpiece. 
In this study, the effect of process parameters on orbital EDM was examined. Some of the parameters investigated were the orbit radius, contact area, pulse interval and translation speed. In terms of MRR, it was shown that there exists an optimum power density at which MRR is maximized.

König et. al. [29] studied the effect of power density for different pulse intervals and contact areas. All other parameters remained constant. It was found that by varying the pulse interval, an optimum was achieved resulting in maximum MRR. These results are shown in Fig. 2.11.

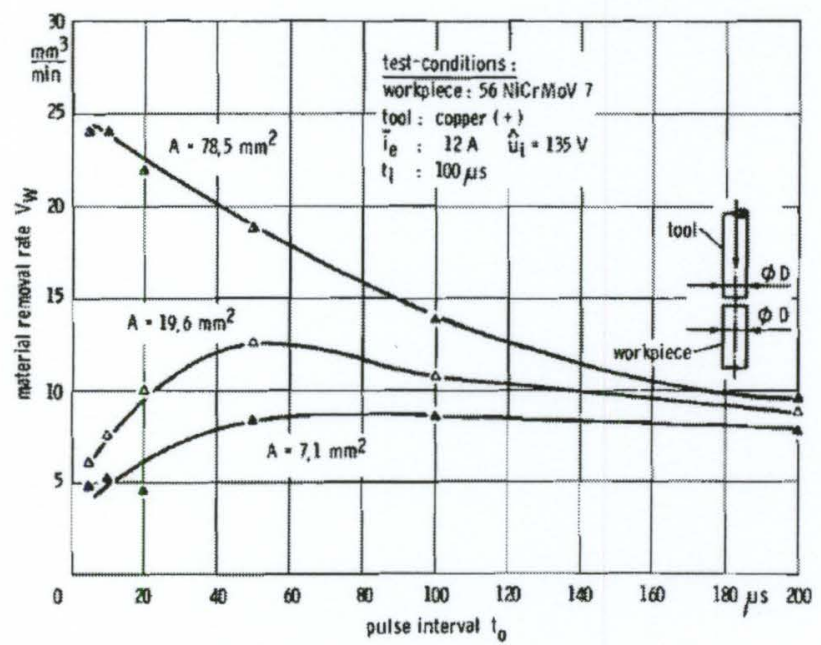

Figure 2.11: MRR vs. Pulse Interval at Different Machining Areas [29]

Tool speed is another parameter that affects the MRR in orbital EDM. Test results showed that increasing the speed of the tool in the xy-plane resulted in an increase in MRR attributed to improved flushing conditions. Furthermore, increased tool speeds resulted in lower relative wear [29].

Staelens and Kruth developed a computer integrated machining strategy for orbital EDM [30]. This work dealt with semi-finishing and finishing of a workpiece rough machined by die-sinking EDM. To minimize machining time, process parameters were determined as the eccentricity of the orbit increased with time. Decreases in 
machining power were achieved by reducing the discharge duration. Only when the discharge duration reached its lower limit $(0.2 \mu \mathrm{m})$ was the current reduced.

Initial tests in [30] showed that circular orbiting with a cylindrical tool achieved much larger MRR than when orbiting with square or rectangular tools. However, when a square orbit was used, the square and rectangular tools outperformed the cylindrical tools. Therefore, a technique was developed in which the circular orbit was modified on-line for square and rectangular tools. During machining, each revolution of the orbit is broken into angular sectors. The maximum eccentricity of these sectors is determined. If a sector has a smaller eccentricity than the maximum of its predecessor, then the angular speed of the orbit is set to zero, resulting in lateral motion. For square and rectangular tools, this results in more efficient machining. Machining with the modified circular orbit using square and rectangular tools produced similar results to machining with a square orbit. Results for the cylindrical tool remained the same, as the orbit was unmodified. Since the modified circular orbit is determined automatically on-line, the orbit geometry can be optimized without user intervention.

Sanchez et al. investigated the dimensional accuracy of orbital EDM for finishing operations [31]. To accomplish this, five different strategies were tested. Based on the strategy and the desired peak-to-valley surface roughness, the radii of consecutive orbits were determined. Once the orbit radii were determined, the initial roughing depth and the dimensions of the finishing electrode could be calculated.

Testing of the different strategies showed that the desired surface roughness was obtained along the side walls of the cavity, but not along the bottom surface. It was suggested that this was a result of thermal effects based on temperature measurements made on both the tool and the workpiece [31]. Therefore, a finite element model was developed using the temperature measurements and material properties as input. The model simulated the thermal expansion of both the tool and workpiece. 
Results from the simulation showed that thermal effects were of the same order as the undercut in the finishing strategy. Therefore, it was recommended that thermal effects be considered in addition to choosing the appropriate strategy for orbital EDM.

Wang and Shan suggested a geometric method of improving the geometric accuracy in orbital EDM [32]. It is suggested in this work that previously developed compensation models for orbital EDM are sufficient only when using simply shaped tools. When the shape of the tool is a freeform surface, a different approach is required. In this technique, the tool is designed based on the cavity geometry and the orbit used. Given this data, the appropriate die-sinking tool can be offset to achieve the orbital tool. Depending on the orbit motion used, the offset may be non-uniform. The actual offset is performed on a set of points which are the vertices of the mesh representing the appropriate die-sinking tool. Normals at these points and the direction vectors of the orbit are used in the computation of the compensated orbital tool.

\subsection{Innovative Tooling Concepts for CNC EDM}

Innovative tooling concepts for CNC EDM is an area of research which requires much attention. Due to the removal mechanism in EDM, tooling possibilities for this process are endless. The combination of standardized tooling and CNC capabilities can improve process efficiency and reduce tooling costs.

Bayramoglu and Duffill $[33,9]$ categorized the tooling used in CNC EDM into four groups; 3D form tools, ball ended/cylindrical tools, plate tools and frame tools. Fig. 2.12 shows these groups of tools graphically. 


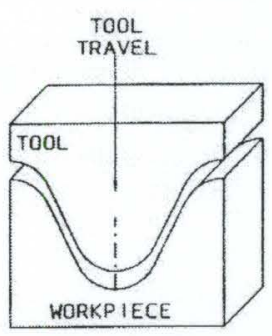

30 FORMTOOL

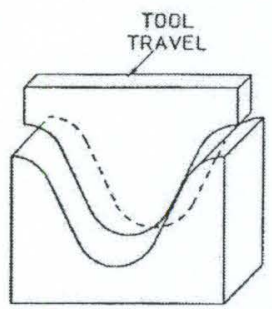

PLATE TOOL

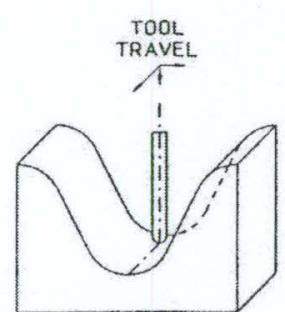

BALL. ENOED CYLINDRICAL TOC

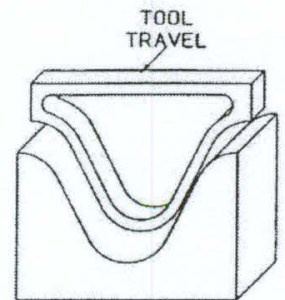

FRAME TODL

Figure 2.12: Tool Types used in CNC EDM [33]

In addition to categorizing the tool types use in CNC EDM, Bayramoglu and Duffill discuss limitations of each tool type in terms of cavity geometry. It is suggested that $3 \mathrm{D}$ form tools and ball ended/cylindrical tools may be used to machine all types of cavities. However, frame and plate type tools are limited to simple cavities. Due to the geometric design of the tool, they are unable to machine sculptured surfaces.

One of the earliest attempts at novel tooling for use with a CNC EDM was proposed by Saito et al. [34]. In this work, frame type tools were used for the machining of 3D cavities. The main benefit of using frame type tools is the minimal amount of material which needs to be removed. Only the outermost walls of the cavity require machining. The rest of the material remains intact and can be removed as a slug from the workpiece upon the completion of machining. Because of this, the machining times in this process can be significantly lower than those in die-sinking EDM. Fig. 2.13 shows the sequence of steps in the machining of a 3D cavity using frame type tools where the number indicate the machining sequence. Also shown is the removed stock material which remains intact after machining. 


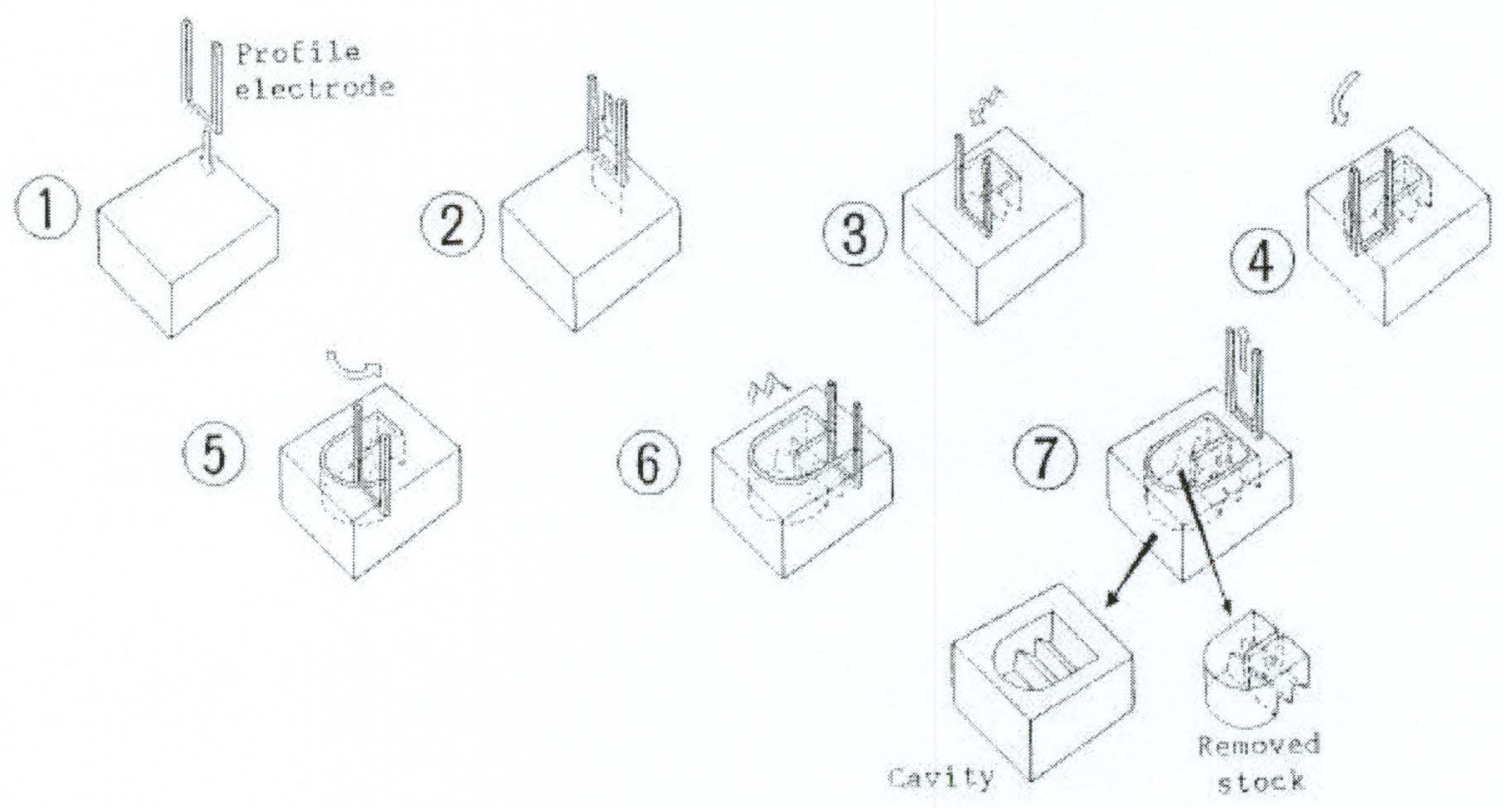

Figure 2.13: CNC EDM using Frame Type Tools [34]

This type of machining requires relative motion between the tool and the workpiece and tool rotation about the z-axis. In order to achieve this, a CNC EDM machine was developed which was capable of 4-axis simultaneous interpolation [34]. The motion control was hard coded and a post-processor converted and transferred the commands to $\mathrm{NC}$ tape.

Another feature of the EDM machine tool developed by Saito et al. was automated tool changing. It was suggested that the combination of a tool changer and standardized tooling would help with geometric inaccuracies due to tool wear [34]. It wasn't until later that wear for this machining process would be characterized.

Bayrmoglu and Duffill further investigated the use of frame type tools in CNC EDM [33]. They performed a systematic study in which cavities with both linear and circular features were machined. These tests allowed them to capture many aspects of the process including machining times and relative electrode wear (REW). 
Frame type tools and 3D form tools were used to machine block shaped cavities of different sizes. The machining times were measured for each block size and it was shown that for volumes greater than $27,000 \mathrm{~mm}^{3}$ frame type tools outperform 3D form tools [33]. Average surface roughness was also measured and showed that the use of frame type tools produced a smoother surface than the use of 3D form tools.

The MRR and REW were measured based on motion (linear/circular) and tool size. In terms of motion, it was found that linear cutting had a higher MRR and a lower REW than circular cutting [33]. When the tool is performing a circular cut, the amount of machining varies radially. The portion of the tool tracing the profile during rotation does much more machining than the portion of the tool located at the centre of rotation. Therefore, this portion of the tool experiences a large amount of wear. During linear cutting, all sides of the frame tool experience equal machining and the wear is distributed more evenly.

Another important issue, which was not addressed in Saito's work, is the deflection of the remaining stock. A simulation corresponding to the worst case scenario resulted in a deflection of $0.001 \mu \mathrm{m}$ [33]. Considering the typical size of a spark gap $(10-100 \mu \mathrm{m})$, this effect was negligible.

In 2004, Bayramoglu and Duffill conducted a similar study on the use of plate tools in CNC EDM [9]. Plate type tools are similar to frame tools, but do not contain a void. Therefore, these tools must remove more material. However, due to the increase in surface area, plate tools can capacitate more electrical power than frame type tools and thus have higher MRR.

MRR was measured at different currents while the tool size $\left(3 \mathrm{~cm}^{2}\right.$ and $\left.12 \mathrm{~cm}^{2}\right)$ remained constant. Increasing currents resulted in higher MRR. However, the MRR for the smaller tool began to approach a maximum suggesting an optimum current density, which was not attained due to generator power limitations [9]. 
Bayramoglu and Duffill also studied the REW and found that larger tools experience less wear than the smaller tools as current increases. It is suggested that this is due to the large difference in current density between the tool sizes. The results of the MRR and REW tests are shown in Fig. 2.14.
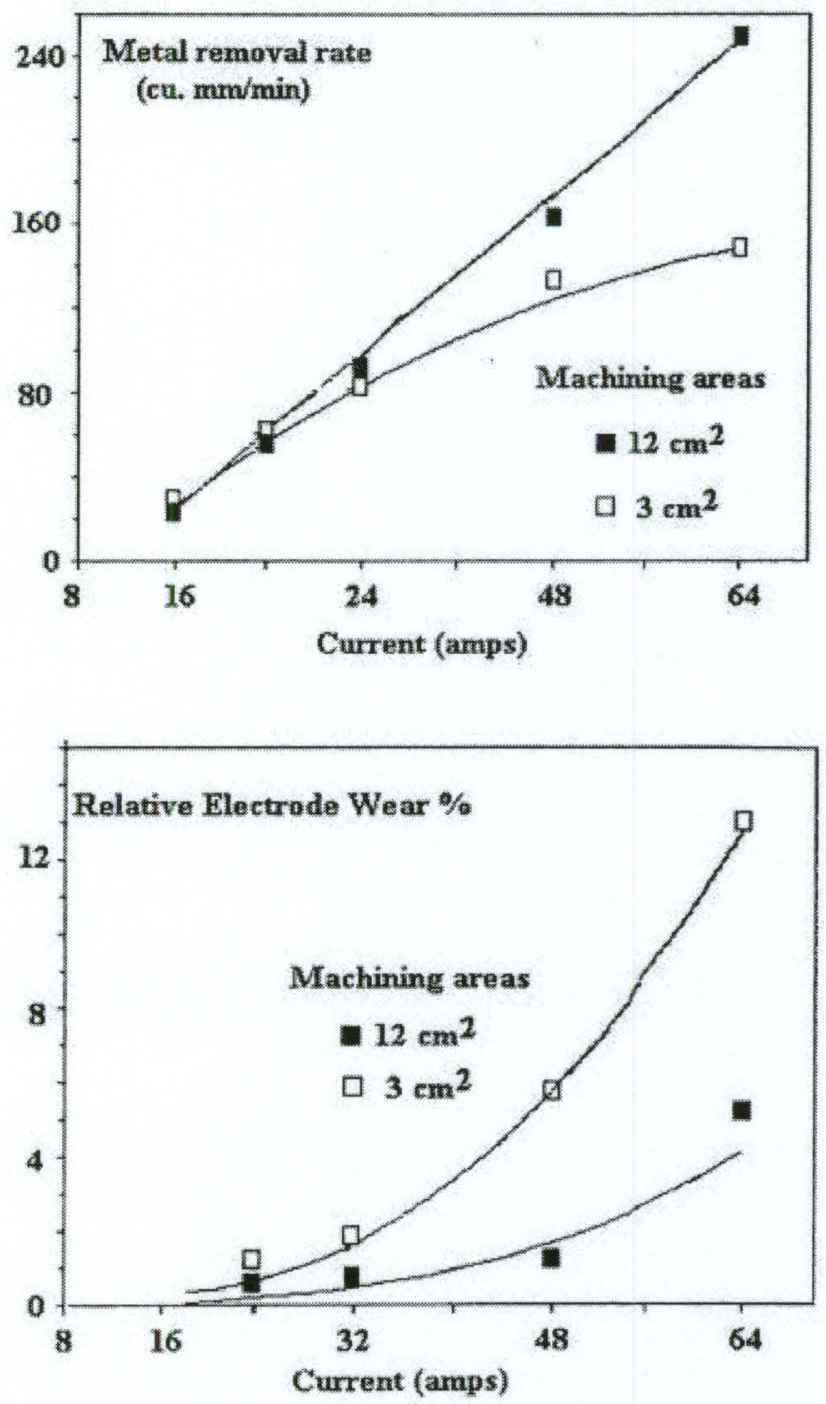

Figure 2.14: MRR vs. Current with Constant Tool Size [9]

Plate type tools were compared to 3D form tools in this study. It was shown that the 3D form tools had a higher MRR. However, when the average surface roughness was measured, plate tools produced better results at all generator settings [9]. 
The most recent innovative tooling concept was developed by Ziada and Koshy [35]. They proposed the use of rotating curvilinear tools for CNC EDM sinking of polygonal shapes with sharp corners. Relative motion between the tool and the workpiece along with constant rotation of the tool facilitate flushing during machining. The tool shape proposed for this machining strategy a modified Reuleaux triangle. The tool is shown in Fig. 2.15 along with an unmodified Reuleaux triangle.

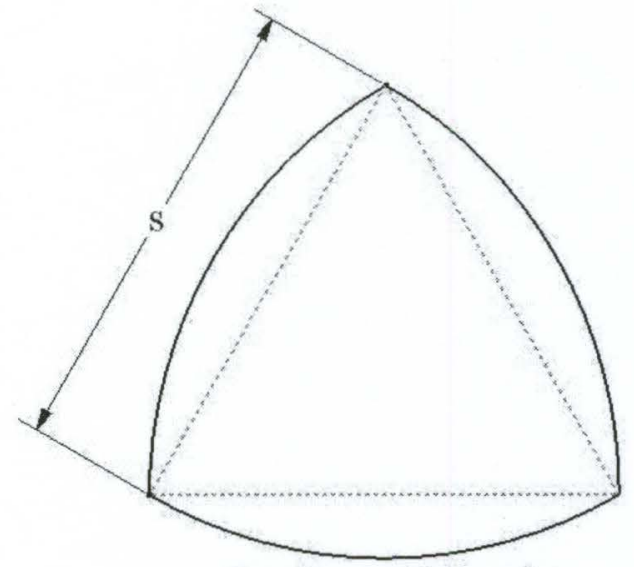

Reuleaux Triangle

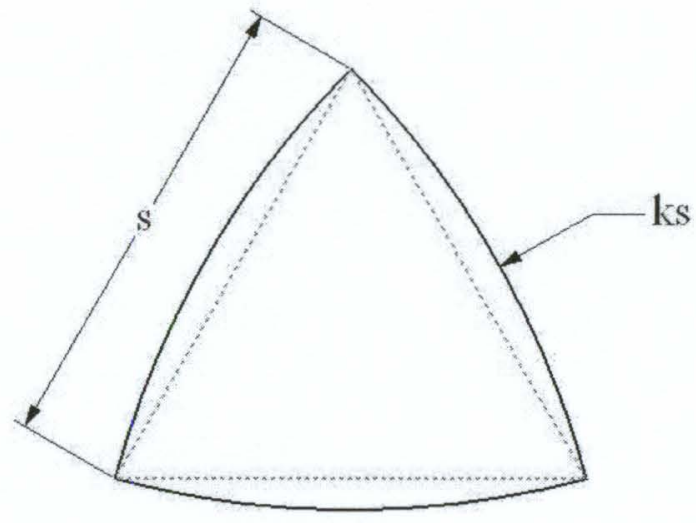

Modified Reuleaux Triangle

Figure 2.15: Unmodified and Modified Reuleaux Triangles (Adapted from [35])

The radii of the sides composing the Reuleaux triangle restrict it to the machining of sharp corners with interior angles of $120^{\circ}$ or greater. Therefore, in order to machine sharp corners of $90^{\circ}$ the radii of the arcs composing the Reuleaux triangle were changed from $s$ to $k s$, where $k$ is a constant equal to 1.93 .

Tool path generation for the machining of polygonal shapes with sharp corners using a modified Reuleaux triangle may be described by parametric equations. These equations are elliptical in nature and are listed below [35]. $x_{p}$ and $y_{p}$ are the centroid of the tool, $s$ is the distance between vertices, $\alpha$ is the angular orientation of the tool with respect to the edge being machined, $\beta$ is a correction factor and $n$ is the number of sides comprising the polygon. 


$$
\begin{aligned}
& x_{p}=\frac{s}{6}(-3+\sqrt{3} \cos \alpha+3 \sin \alpha)+\beta s\left(\frac{60-\alpha}{180 / n}\right) \\
& y_{p}=\frac{s}{6}(3-\sqrt{3} \cos \alpha-3 \sin \alpha)
\end{aligned}
$$

The driving parameter in the equations is $\alpha$. Varying alpha and applying translations/rotations to the equations allows for the machining of regular and irregular polygons with sharp corners. Fig. 2.16 shows some cavities machined using a modified Reuleaux triangle type tool.
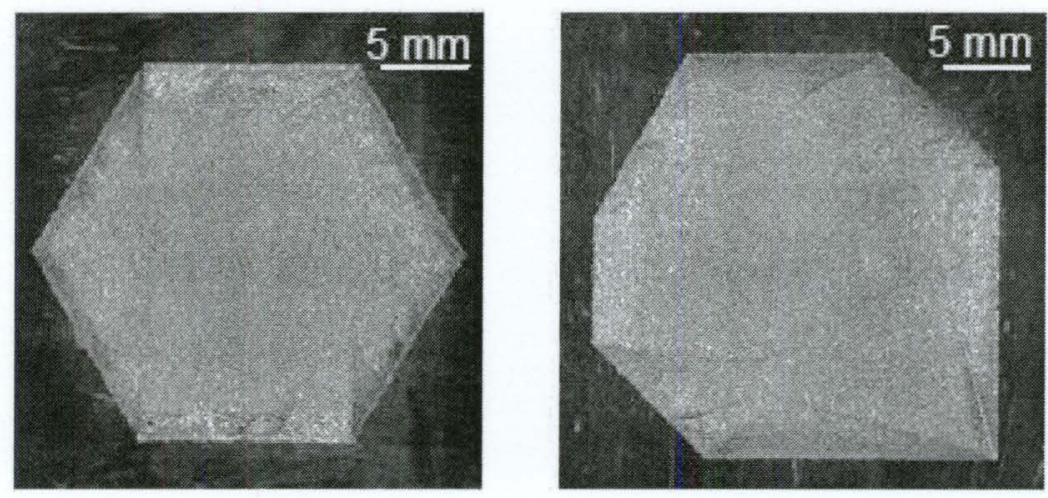

Figure 2.16: Cavities Machined by a Rotating Reuleaux Triangle (Adapted from [35])

The development of innovative tooling concepts for CNC EDM has great potential. However, there is lack of research in this area due to the focus on tool wear compensation for ED milling with cylindrical tools. To this end, innovative tooling concepts for CNC EDM are explored in this work with the innovative development of an oblong tool set for ED milling. By exploiting the increased frontal area of the oblong tools, as compared to conventionally used cylindrical tools, the process performance may be maximized. 


\section{Chapter 3}

\section{Tool Set}

In this chapter, a novel tool set developed for ED milling is introduced. Motivation for developing the tool set and a list of criteria which it must satisfy are presented. The function of each tool in the set is described along with parameters defining the size of the tool.

\subsection{Motivation}

In ED milling, cylindrical tools are traversed along a programmed path to machine the desired shape. The use of cylindrical tools limits the tool in size and power capacity. It is reported in the literature that there exists an optimum current density $^{1}$ at which a maximum MRR is achieved, while maintaining a stable process $[5$, $23,9,36,30,37]$. This means that a larger tool has a higher current at which the process remains stable. The higher current is accompanied by a higher MRR. Fig. 3.1 shows the relationship between MRR and current density. At maximum MRR, the current density is optimal.

\footnotetext{
${ }^{1}$ Current density is defined as $\frac{I}{A}$, where I is current and A is the frontal surface area of the tool engaged in machining.
} 


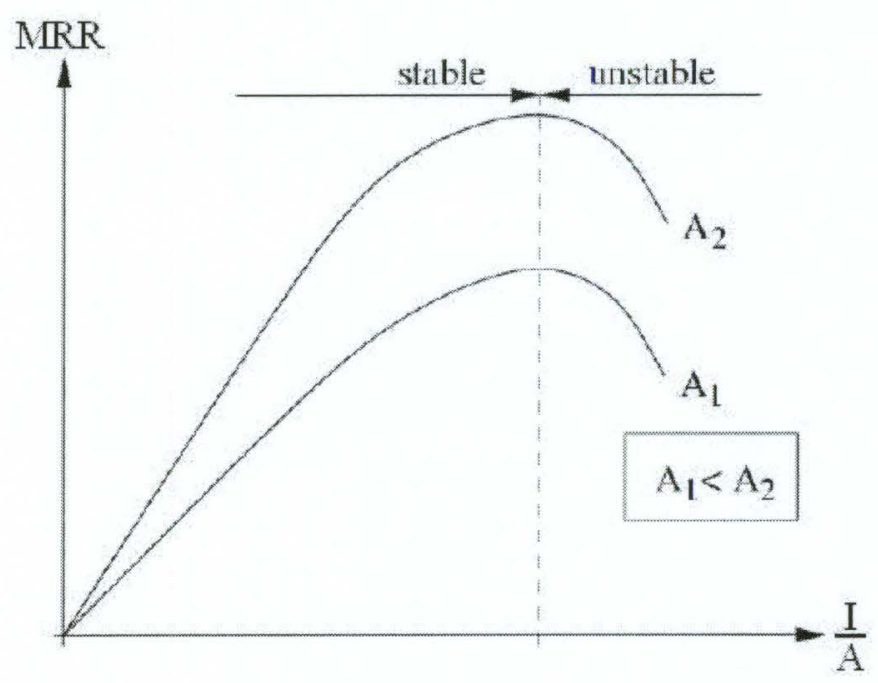

Figure 3.1: MRR vs. Current Density [36]

Also shown in Fig. 3.1 is the effect of increased area. Area $A_{2}$ is greater than area $A_{1}$ and thus has a higher MRR. This is due to the higher current required to maintain optimum current density.

Though the size of the cylindrical tools used in ED milling limits power capacity, it improves flushing ability. Since the size of the tool is generally much smaller than the cavity being machined, there is ample space available for the recirculation of dielectric fluid and thus flushing of debris [35]. This excess room also makes it possible to use external jet flushing in the machining of deep cavities.

The tools currently used in ED milling are unfavourable for machining sharp corners. To machine the approximation of a sharp corner using a cylindrical tool, the tool must be extremely small. Referring to previous arguments on tool size, this is undesirable.

Another advantage of cylindrical tools is their cost. Since tooling generates a large portion of the costs associated with EDM, this is an important benefit. Cylindrical tools are readily available which eliminates the lead time required for tool manufacture associated with die-sinking. 
Based on the arguments presented above, a list of criteria for the development of a tool set is presented:

1. Large surface area leading to higher power capacity

2. Sufficient flushing

3. Simple in shape and cost effective

4. Ability to machine sharp corners

\subsection{Development of a Tool Set}

The first criterion requires a set of tools with large surface area. This facilitates a higher power capacity. In die-sinking, the power capacity of the tools is maximized since the tools are manufactured to be just slightly undersized with respect to the machined cavity. However, the size of the tools restricts flushing and the process becomes unstable at high current densities.

The next criterion refers to sufficient flushing. In this sense, a small set of tools is desirable. However, as previously stated, these tools would be limited in power capacity. Furthermore, the large amount of wear that the tools would sustain is undesirable. Another consideration is the use of external jet flushing which requires ample room for the jet stream to penetrate the gap. Therefore, the size of the tools in the set must be between cylindrical tools and die-sinking tools.

The third criterion suggests that the tools must be simple in shape and cost effective. Manufacturing costs and lead times associated with the tooling used in EDM are dictated by the complexity of the tool. Wire electrodes and cylindrical tools are the simplest used in EDM. Therefore, the tools in the set should closely resemble cylindrical tools or their geometric variants. 
The final criterion states that the set should contain a tool which can machine sharp corners. Strategies have been developed for the machining of sharp corners using planetary EDM. However, these strategies require the use of a tool similar to those used in die-sinking EDM, only smaller. This requires the fabrication of a special tool and when combined with ED milling, an additional NC program. Therefore, the tool set will contain tools capable of machining sharp corners.

Both the cylindrical tools and die-sinking tools meet some, but not all of the criteria described above. Therefore, a new set of tools is required which satisfies all of the criteria. The set of tools conceived in this work is shown in Fig. 3.2.

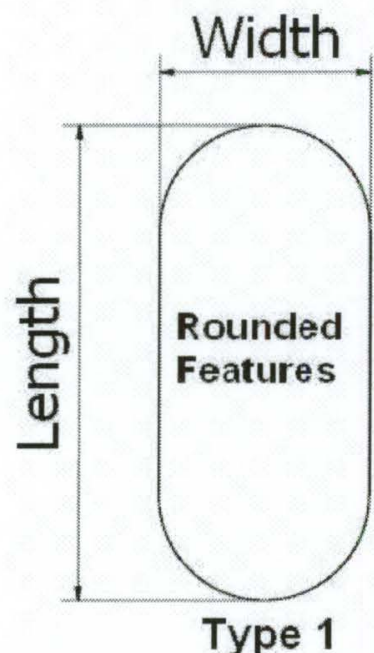

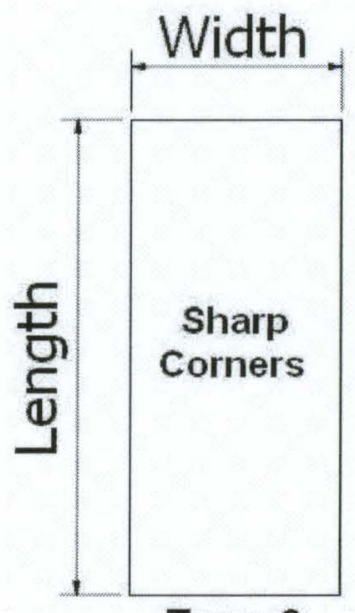

Type 2

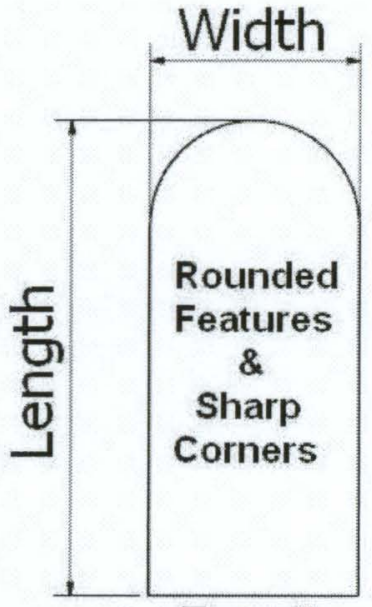

Type 3

Figure 3.2: Oblong Tool Set

This set of tools allows for machining of cavities with rounded and sharp features. It also provides an increase in area and thus, a high power capacity, while offering sufficient flushing. The shape of the tools is simple to reduce manufacturing time and costs. Furthermore, the raw material required is much less than that for die-sinking tools. A comparison of the different tool types, based on the outlined criteria, is shown in Table 3.1. It shows that the tool set presented in Fig. 3.2 is the only one that satisfies all of the criteria. 


\begin{tabular}{lccc} 
Criteria & Die-Sinking Tools & Cylindrical Tools & Oblong Tools \\
\hline Large Area/High Power Capacity & Yes & No & Yes \\
Sufficient Flushing & No & Yes & Yes \\
Cost Effective & No & Yes & Yes \\
Ability to Machine Sharp Corners & Yes & No & Yes
\end{tabular}

Table 3.1: Tool Set Comparison

The reason that this tool set can offer all of these benefits is its geometry which is similar to that of cylindrical tools. The main difference is that these tools have been elongated to provide more surface area while still maintaining, for flushing purposes, a width equal to the diameter of an equivalent cylindrical tool. Another modification is the sharp ends of the tools which can be used for machining sharp corners without the need to change tools or incur additional programming efforts. 


\section{Chapter 4}

\section{Tool Type Selection and Tool Size Calculations}

This chapter presents algorithms for tool type selection and tool size calculation. The algorithms are presented according to the variations in cavity profile. Cavity profiles considered in this work are divided into three groups that are defined in this section. A discussion of the 2D medial axis transform (MAT), which is used in computation of the tool size, is also presented.

\subsection{Profile Definitions}

The cavities considered in this work may be represented by $2 \mathrm{D}$ closed-loop profiles. A closed-loop profile is one which contains no disjoint curves. In other words, all curves contained in a closed-loop profile are connected to each other ${ }^{1}$. Based on their profiles, the cavities considered may be divided into three groups. Before the groups are presented, a discussion of geometric continuity and concavity is required.

\footnotetext{
${ }^{1}$ The term 'curves' refers to lines, circular arcs, b-splines, etc.
} 
Geometric continuity is a measure of smoothness between joined curves and the curves themselves. Higher orders of geometric continuity result in smoother junctions. $\mathrm{G}^{0}$ continuity occurs when neighbouring curves are connected by their endpoints. This type of continuity ensures that the profile forms a closed-loop. $\mathrm{G}^{1}$ continuity occurs when neighbouring curves are tangent at their endpoints. It should be noted that $\mathrm{G}^{0}$ continuity is implied for a profile which is $\mathrm{G}^{1}$ continuous.

Tangent continuity in concave regions of a profile provides the basis for profile classification. In this sense, concavity is determined with respect to the interior of the profile. Therefore, concave regions of the profile open away from its interior, while convex regions open towards it. Concave regions exist when a tangent along the profile lies outside of the profile itself. Fig. 4.1 shows how concavity is defined in this work along with examples of tangent continuity and discontinuity.
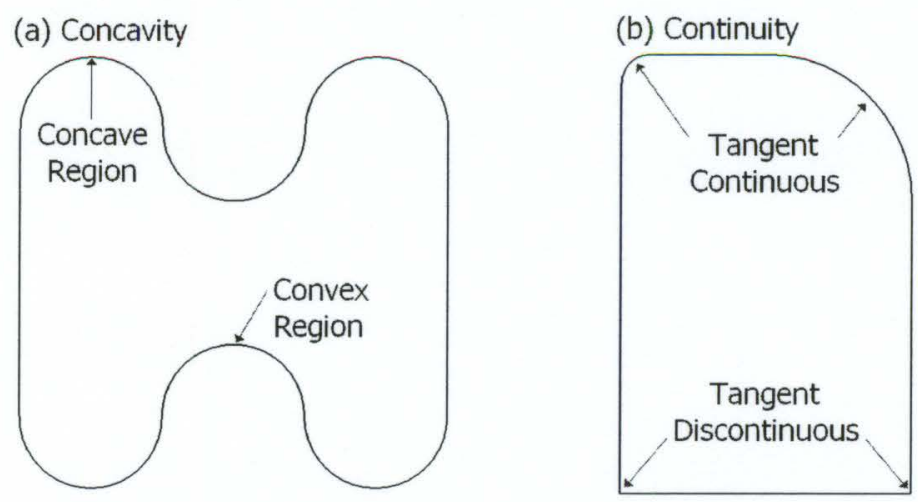

Figure 4.1: (a) Concavity and (b) Tangent Continuity

Classification of profiles also depends on geometric composition at concave regions of the profile. Geometric composition refers to the set of curves contained in a profile, which may include lines, circular arcs, b-splines, etc. Based on the geometric composition and tangent continuity at concave regions of the profile, profiles may be expressed by the following groups. 
Profile Type 1: Contain only concave regions which are $\mathrm{G}^{1}$ continuous.

Profile Type 2: Contain only concave regions which are $\mathrm{G}^{0}$ continuous and composed of only lines.

Profile Type 3: Contain at least one concave region which is $\mathrm{G}^{0}$ continuous and composed of linear and non-linear curves.

Profiles of type 1 are tangent continuous along concave regions of the profile. Therefore, such profiles are free of sharp interior corners. Fig. 4.2 shows a concave profile belonging to this group.

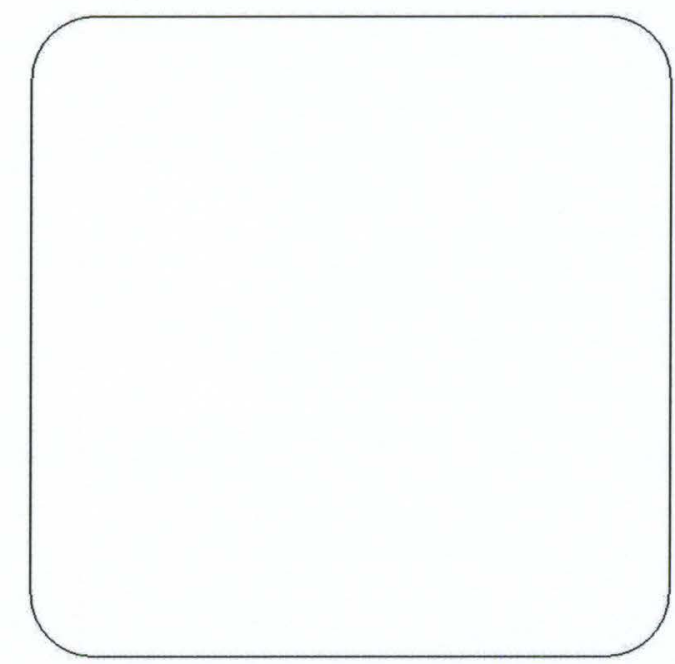

Figure 4.2: Profile Type 1 Example

Profiles of type 2 contain concave regions which are tangent discontinuous and consist only of lines. Therefore, concave regions on these profiles are not smooth and include sharp corners. Due to tool design, the sharp corners are limited to angles greater than or equal to $90^{\circ}$. Fig. 4.3 shows a concave profile of this type. 


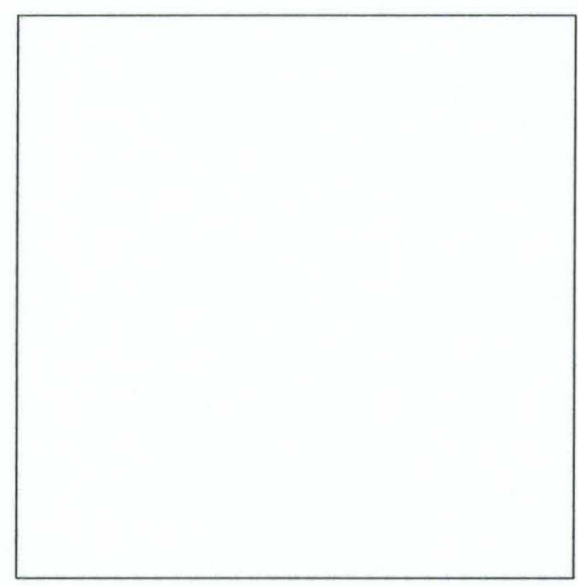

Figure 4.3: Profile Type 2 Example

When a profile contains concave regions which are composed of both linear and non-linear curves and at least one concave region which is tangent discontinuous, the profile is of type 3. Such a profile contains sharp corners greater than or equal to $90^{\circ}$. It should be noted that only one concave region must meet this criteria for the profile to be classified as type 3. Fig. 4.4 shows a concave profile of type 3 which contains two tangent discontinuities.

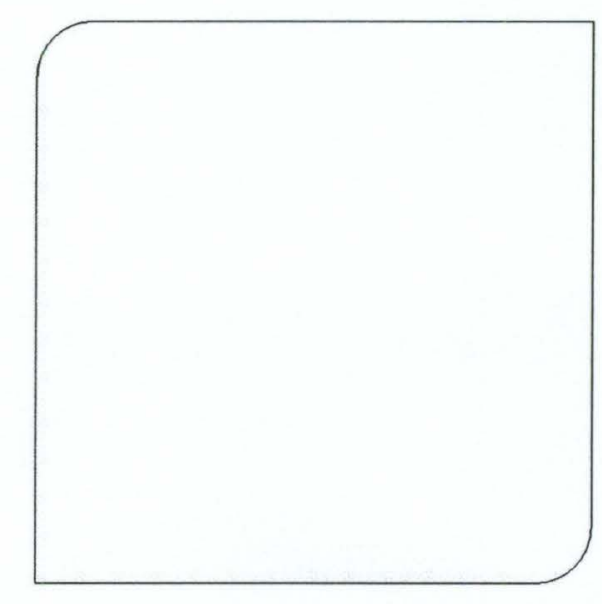

Figure 4.4: Profile Type 3 Example

Division of the cavity profiles into groups provides the structure for the remaining work. Each of the algorithms will be presented according to profile groups. The first of these algorithms is tool type selection. 


\subsection{Tool Type Selection}

The tool set developed in this work contains three tool types. Given the profile of a cavity, the appropriate tool type must be chosen. In order to automate this process, each tool is associated with a profile type. An algorithm which classifies the profile type of a given cavity is presented. By association, the tool type can then be selected. Table 4.1 shows the association between profile types and tools.

\begin{tabular}{cc} 
Profile Type & Associated Tool Type \\
\hline \hline Profile Type 1 & Tool Type 1 \\
Profile Type 2 & Tool Type 2 \\
Profile Type 3 & Tool Type 3
\end{tabular}

Table 4.1: Association between Profile Type and Tools

The pairing of profile types and tool types is a result of geometric similarity. For instance, profiles defined as type 1 include concave regions of $\mathrm{G}^{1}$ continuity. This means that the tool required to machine such profile may be at most $G^{1}$ continuous. Tool type 1 is $\mathrm{G}^{1}$ continuous and thus paired with profile type 1 .

Concave regions in profiles of type 2 are $\mathrm{G}^{0}$ continuous and consist of only lines. Therefore, the tool used to machine such profiles must be $\mathrm{G}^{0}$ continuous. This ensures that the tool will have the tangent discontinuities required for the machining of sharp corners. Based on this criterion, tool type 2 is paired with profiles of type 2 .

Profiles described by profile type 3 contain concave regions with tangent discontinuities and both types of curves. Unlike type 2 profiles, profiles of type 3 include tangent continuous concave regions. Therefore, the tool must contain regions of tangent continuity and discontinuity. Tool type 3 has both of types of regions and thus is paired with type 3 profiles. Profile/Tool pairs are shown in Fig. 4.5. It is to be noted that tool type 2 may be paired with profiles of type 1 and 3 . However, this is usually accompanied by a decrease in tool size, but not always. 

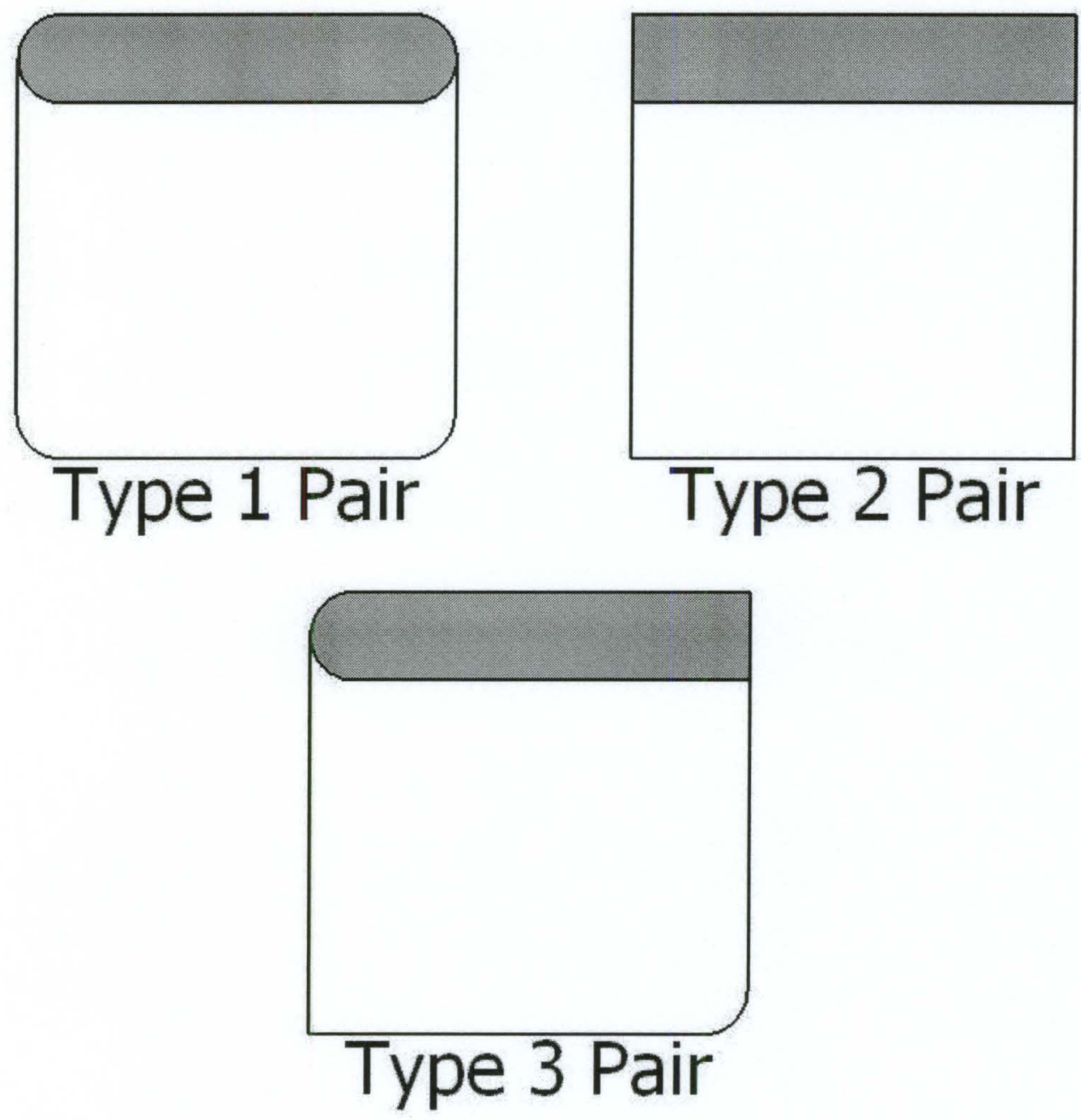

Figure 4.5: Profile/Tool Type Pairs

To select the appropriate tool type for a given profile, the profile must first be classified. This is achieved by stepping through the profile curve-by-curve. At each curve, the curve type along with its concavity and continuity relationship to its successive neighbour are recorded. Based on the results of this search, an appropriate tool is suggested. Fig. 4.6 shows a flow chart representation of this algorithm. 


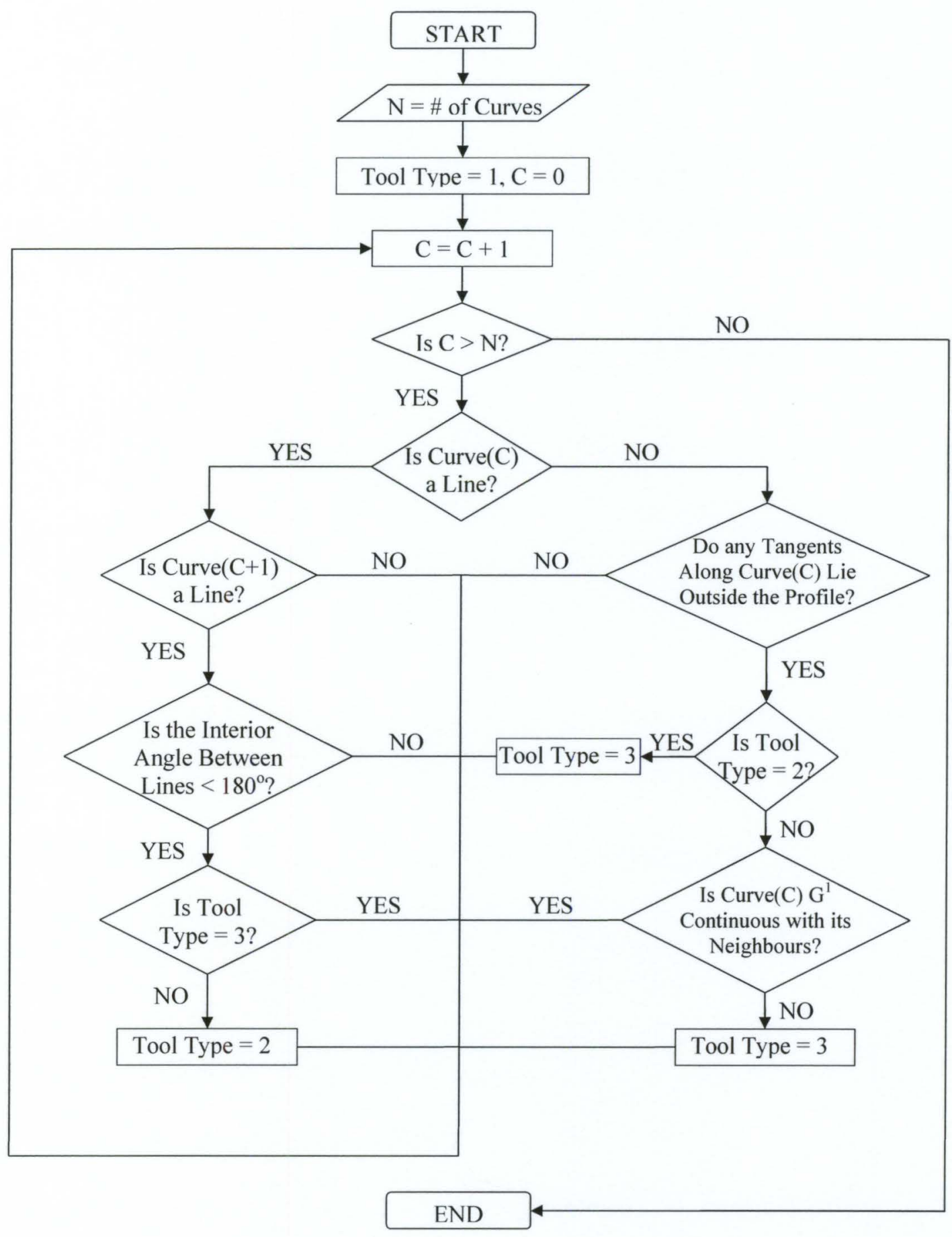

Figure 4.6: Tool Type Selection Algorithm 


\subsection{Medial Axis Transform (MAT)}

The MAT is a method of geometric representation which uses circles (2D) or spheres (3D) as primitives. It was first introduced in 1967 by Harry Blum [38]. In this work, he used the transformation to describe complex profiles of biological shapes. It has since been used for many applications including image analysis, mesh generation, mold design and tool path planning [39].

The concept behind the MAT is that any $2 \mathrm{D}$ or $3 \mathrm{D}$ geometry can be represented by a medial axis (MA) and its accompanying radius function [40]. Since this work is focussed on the machining of 2D cavities, the 2D MAT will be used. For a 2D profile, the MA is $2 \mathrm{D}$ and the radius function defines the radii of circles centred on the MA.

The MA is a set of points defined by the centres of maximal inscribed circles which represent the profile boundary. The radius function is a continuous function describing the radii of the circles located on the MA. Combining the MA and radius function result in the MAT $[39,41]$. A 2D profile and its MAT are presented in Fig. 4.7. The profile, the MA, and the circles defining the profile boundary are shown.

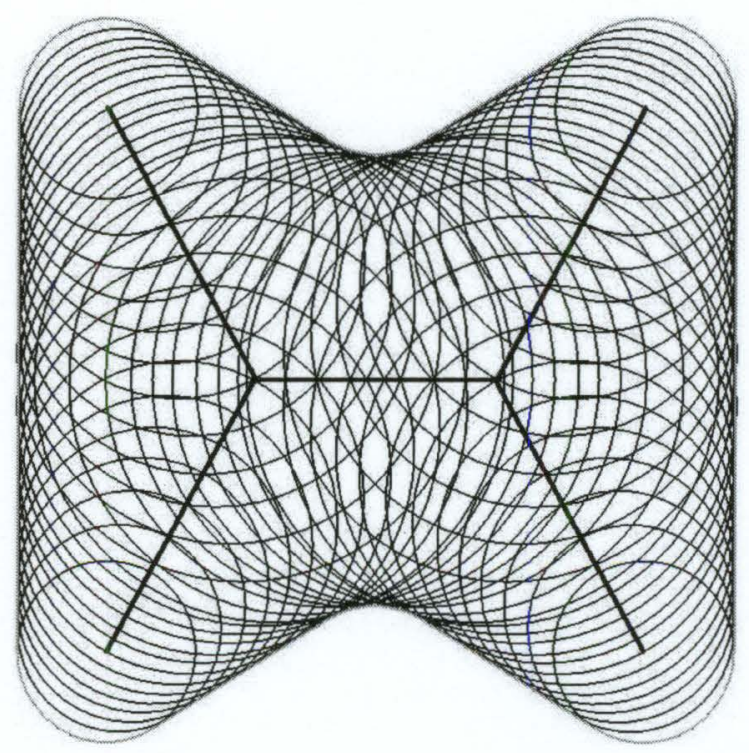

Figure 4.7: Medial Axis Transform of a 2D Profile 
Many algorithms have been presented for computing the 2D MAT of a given profile. VRONI [42] is the algorithm used to determine the MAT in this work. In this algorithm, lines are represented by three parts, an open line segment and two endpoints. Curves are represented as linear approximations [42].

The algorithm begins by inserting four "dummy" points forming a bounding box around the profile. Then the endpoints of the line segments are inserted randomly. After each point is inserted, the Voronoi diagram is computed. Once the Voronoi diagram has been completed for the endpoints, the line segments are added incrementally and the Voronoi diagrams are computed again. Once all of the line segments have been inserted, the closed profile is complete and the Voronoi diagram can be computed [42]. The MA and radius function are then calculated ${ }^{2}$. Once the MAT has been determined, the tool size calculations can be performed.

\subsection{Tool Width Calculation}

The algorithm presented in this section computes the maximum permissible tool width based on the accessibility of cavity features. In other words, the maximum tool width is calculated such that all features of the cavity may be machined with a single tool. Therefore, the value determined by the algorithm constrains the tool size meaning that only a width less than or equal to the calculated value may be assigned as the tool width.

Tool width constraints are defined by a feature of minimum size. For each profile type, the feature of minimum size is defined differently. Therefore, a methodology for computing maximum tool width will be presented for each type of profile. A general algorithm is also presented which chooses the proper methodology.

\footnotetext{
${ }^{2}$ VRONI determines the MAT as a subset of the Voronoi diagram. [43]
} 


\subsubsection{Tool Width Calculation - Profile Type 1}

The concave regions in profiles of type 1 are $\mathrm{G}^{1}$ continuous. Therefore, the feature of minimum size is defined by the feature with the smallest internal radius. In the absence of a narrow passage, this feature must dictate the tool width constraint to ensure that the tool is able to machine the entire cavity. If the tool width is greater than the smallest internal radius, it will not be able to machine the smallest feature. This idea is shown in Fig. 4.8. In (a), the tool is too wide to machine the smallest internal radius while in (b), the tool can machine the feature.

(a)

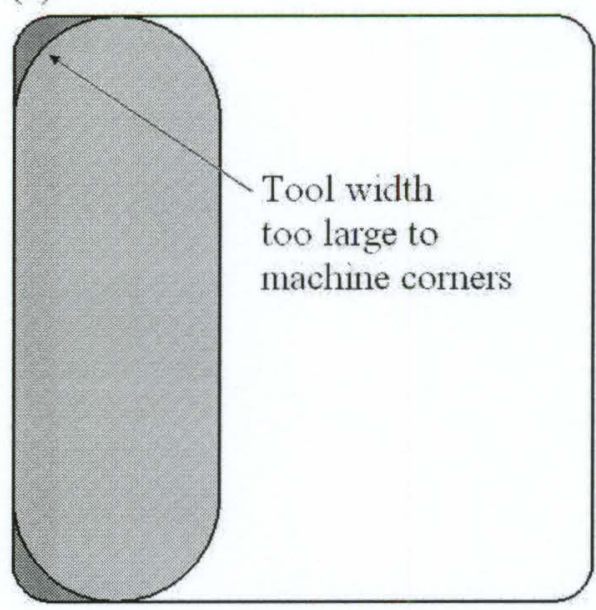

(b)

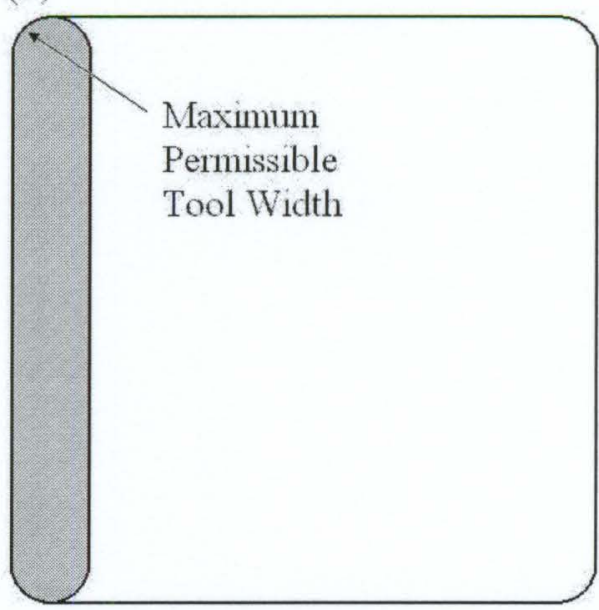

Figure 4.8: Tool Width Constraint Defined by Smallest Corner Radius

In the presence of a narrow passage, both the smallest feature radius and the narrow passage itself must be considered when determining the maximum tool width. The reason for this is again accessibility. In order for the tool to machine every region of the cavity, the tool width must be small enough for the tool to pass through narrow passages. In addition to this, the tool width must still be able to machine the smallest feature. Therefore, in the presence of a narrow passage, the smallest feature and the passage width must be compared. The diameter equivalent to the smallest of these values defines the maximum permissible tool width. 
The MAT can be used to determine the feature of minimum radius and the width of any narrow passages. Due to the nature of the MAT, it also eliminates the need to compare the two values. Searching the radius function of the MAT for a minimum provides the tool width constraint for profiles of type 1 .

\subsubsection{Tool Width Calculation - Profile Type 2}

Cavities of profile type 2 contain tangent discontinuous concave regions composed of lines. Since concave regions in these profiles contain no rounded features, the feature of minimum size is defined by a length rather than a radius. Therefore, the MAT cannot be used for computation of the feature of minimum size.

The feature of minimum length is determined by searching the cavity profile line-by-line in a counter-clockwise direction. In some cases, a direct search will not return an appropriate value. Therefore, before the search may begin some conditions must be checked for along the cavity profile. The result of checking these conditions is removal of lines from the search. A list of these conditions is listed below. The term 'line of interest' refers to the line being analyzed at any given instant of the search.

1. If the line of interest is right of the previous line, both lines are eliminated from the search as they form a convexity.

2. If the line of interest is not perpendicular to either of its neighbours, those neighbouring lines which are shorter than the line of interest are eliminated from the search.

The first condition is established because if the line of interest is to the right of the previous line, the profile becomes convex. This means that a larger tool may be used to simply trace this region of the profile. Consequently, such pairs of lines 
are removed from the search ${ }^{3}$. Fig. 4.9 shows a cavity containing lines which are eliminated due to this condition. The eliminated lines are crossed out by an ' $\mathrm{X}$ '.

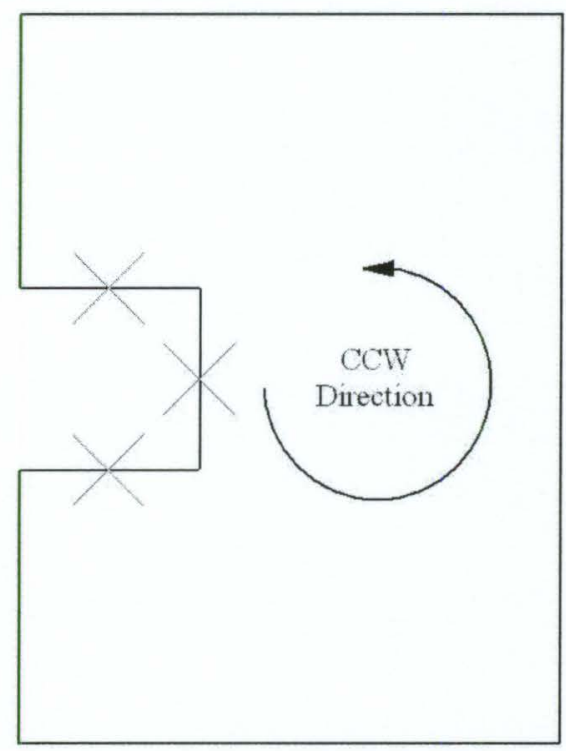

Figure 4.9: Cavity Profile with Lines Removed due to Condition 1

The second condition is a result of tapering. If tapering occurs in a cavity profile, the tool width must be chosen such that the tool can fit into the entire taper. Therefore, the tool width is limited to the length of the line which connects the tapered lines. This line is the line of interest. When tapered lines are longer than the line of interest, they are eliminated from the search without applying condition 2. However, when tapered lines are shorter than the line of interest, condition 2 is satisfied. The tapered lines are removed from the search since they may result in a smaller tool width. As with condition 1, if the tool can fit into the end of the taper, it can simply trace the tapered lines whether they are longer or shorter.

Fig. 4.10 shows (a) the case when tapered lines are removed by condition 2 and (b) the case when they are not. If the tapered lines in (a) are not removed, they remain in the search for the maximum permissible tool width. In (b), the line

\footnotetext{
${ }^{3}$ Non-linear curves are not considered in this search, convex or not.
} 
of interest is shorter than the tapered lines, eliminating the tapered lines from the search without condition 2. The eliminated lines are crossed out by an ' $\mathrm{X}$ '.

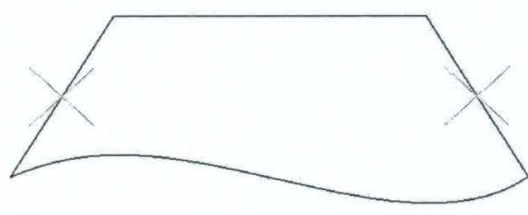

(a)

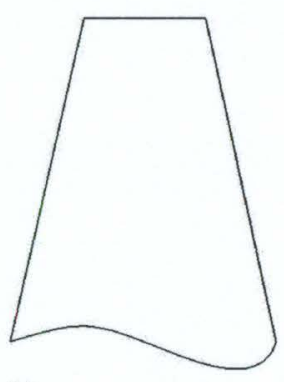

(b)

Figure 4.10: Tapers - (a) Lines Removed and (b) Lines Remain

Once the appropriate lines have been eliminated according to conditions 1 and 2 , the tool width constraint can be calculated based on the feature of minimum length. However, when profiles contain narrow passages, this value must be compared to the width of the narrow passage. The computation of narrow passage width is achieved using a modified medial axis transform (MMAT).

Profiles of type 2 contain sharp corners resulting in branches of the MAT which approach zero. This means that searching the radius function for a minimum will return a value of zero. Therefore, any branches whose radius function approaches zero are eliminated. The result is the MMAT. Fig. 4.11 shows the MA and MMA of a profile.

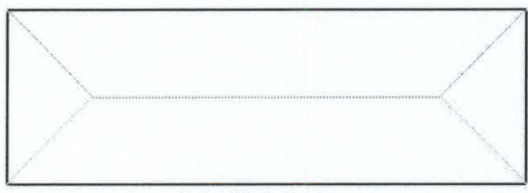

Medial Axis

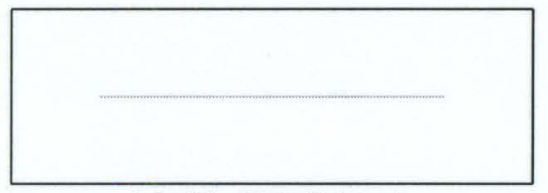

Modified Medial Axis

Figure 4.11: Medial Axis and Modified Medial Axis

A search of the MMAT will return the width of any narrow passages in the cavity. Once this value is computed, it is compared to the feature of minimum length and the smaller of the values defines the tool width constraint. 


\subsubsection{Tool Width Calculation - Profile Type 3}

Profiles of type 3 contain concave regions of all curve types and at least one tangent discontinuity. This means that these profiles may contain features of minimum radius, features of minimum length and narrow passages. Therefore, a combination of the techniques applied for profile types 1 and 2 is used.

Initially, the MMAT of the profile is computed. A search of the radius function returns the smallest of the minimum feature radius and narrow passage width. The feature of minimum length must then be calculated. In order to calculate the feature of minimum length, the profile must be searched curve-by-curve. During the search, regions of the profile which open away from the inside of the cavity profile are ignored. When the search is completed, the width of the narrow passage, the feature of minimum radius and the feature of minimum length are compared. The smallest of the values is the maximum permissible tool width.

\subsubsection{Tool Width Calculation - General Algorithm}

A general algorithm for tool width constraint is presented in Fig. 4.12. It determines the appropriate methodology based on tool type selection. For each methodology, the minimum value is chosen as the tool width constraint. It should be noted that for profiles of type 2 and 3 , the order in steps bears no effect on the end result. 


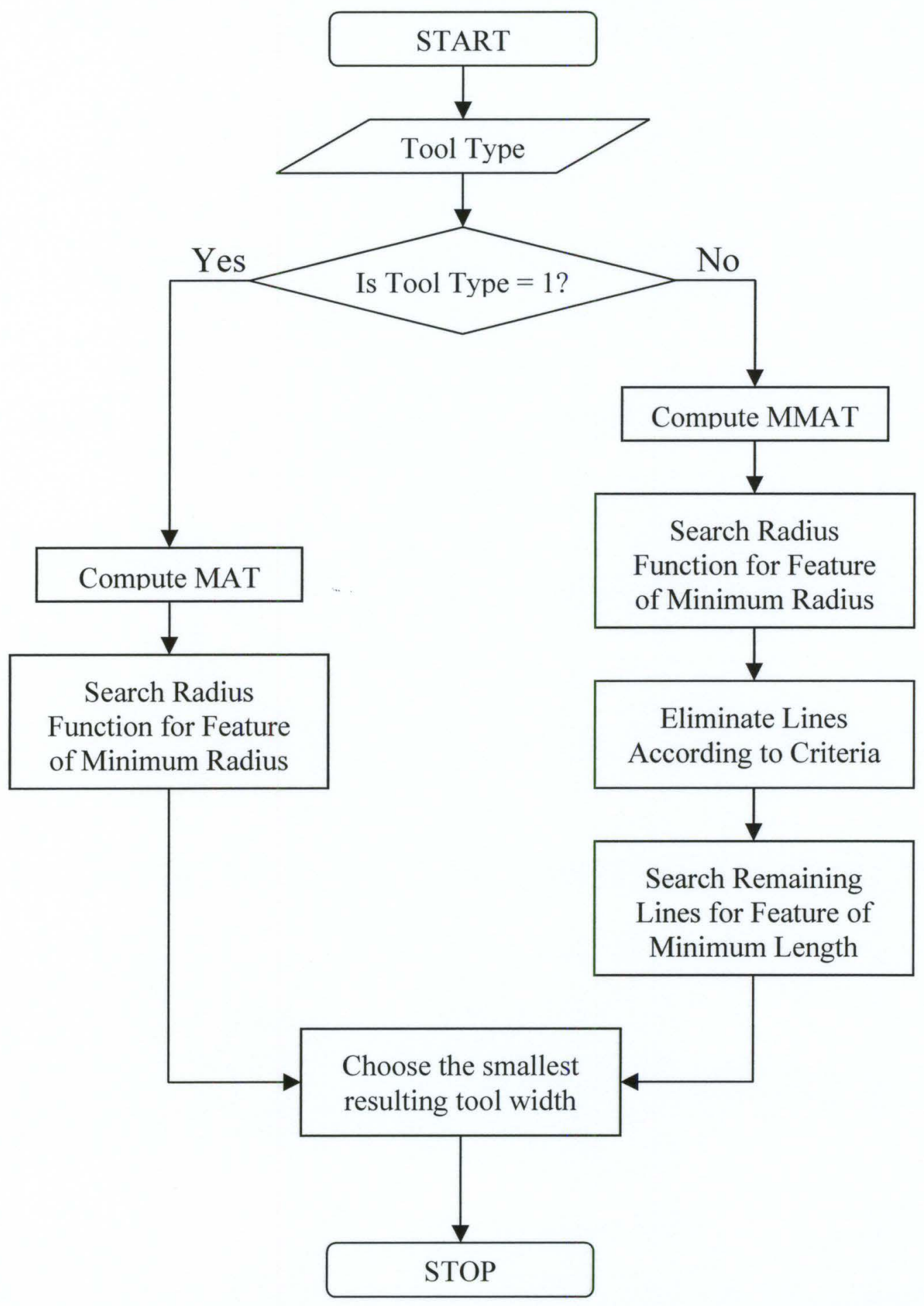

Figure 4.12: Tool Width Algorithm 


\subsection{Tool Length Calculation}

In this section, an algorithm is presented which calculates the maximum permissible tool length. Therefore, any tool whose length is less than or equal to the calculated maximum may be used to machine the cavity, providing that its width is equal to the width used during the calculation.

Three different methods are used in the calculation of the maximum allowable tool length. The first two methods apply to concave regions of the cavity profile while the other applies to convex regions. These methods are similar for each profile type. Therefore, the concept of each method will be presented independent of profile type. The details of the methods as they pertain to each profile type will be presented accordingly.

The first method calculates tool length based on the radius function of the MAT, which was computed during calculation of the maximum allowable tool width. Searching the radius function for a maximum provides the radius of the maximum inscribed circle (MIC). The longest tool, of the defined width, which can fit into the MIC is then determined. Computation of the length is dependent on the tool type as shown in Fig. 4.13. Each tool in Fig. 4.13 has equal width. However, due to differences in geometry, their lengths vary.

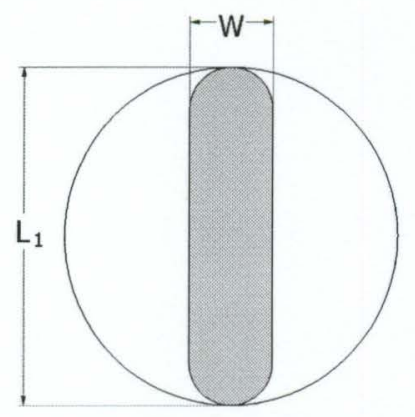

Tool Type 1

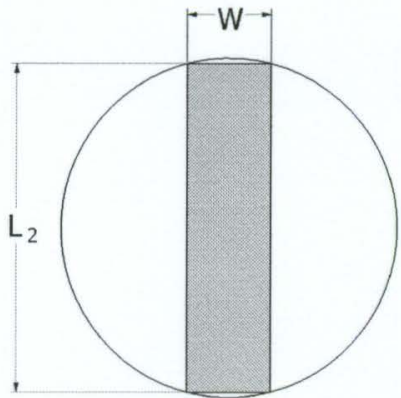

Tool Type 2

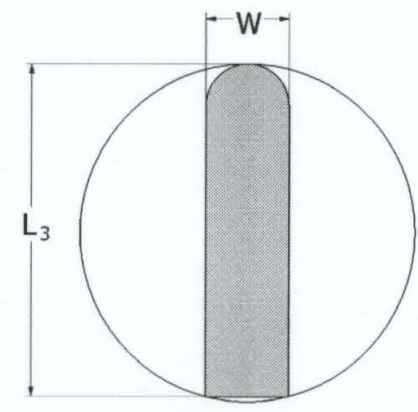

Tool Type 3

Figure 4.13: Tool Length Defined by MIC $-\mathrm{L}_{1}>\mathrm{L}_{3}>\mathrm{L}_{2}$ 
In some cases, a tool length greater than that determined by the MIC may be possible in concave regions of the cavity. This occurs when the cavity contains a large narrow region, thereby requiring another method of calculation. Fig. 4.14 shows examples where a tool larger than that defined by the MIC may be used to machine the cavity.

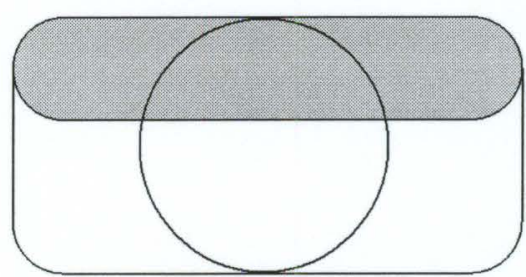

Profile Type 1

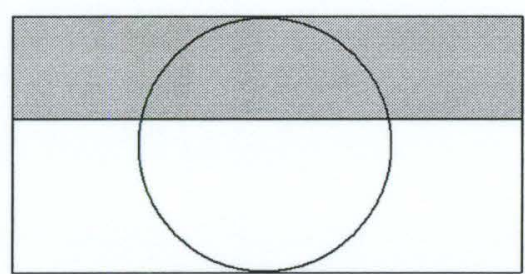

Profile Type 2

Figure 4.14: Tool Length Determined by MIC is not Maximal

Once the tool length defined by the MIC has been determined, the second method for calculating tool length may be considered for profile types 1 and 2 . However, if there exists no lines in the cavity profile whose lengths are greater than the diameter of the MIC, this test need not be performed. In this method, the tool length is determined based on lines contained in the profile offset ${ }^{4}$. In all cases, the appropriate line must be selected.

To determine which is the appropriate line, all lines associated with convex regions of the profile offset must first be removed from the search. Starting with the longest line, the remaining lines are searched one-by-one. The length of each line on the profile offset which is greater than the length of the centre line defined by the MIC corresponds to a possible solution for tool length. More specifically, they represent possible lengths for the centre line of the tool. Since the length of the centre line is determined by this algorithm, the corresponding tool length must be computed based on the tool type. Centre lines for tool type 1 and 2 are shown in Fig. 4.15.

\footnotetext{
${ }^{4}$ The profile is offset by a distance equal to half of the selected tool width.
} 

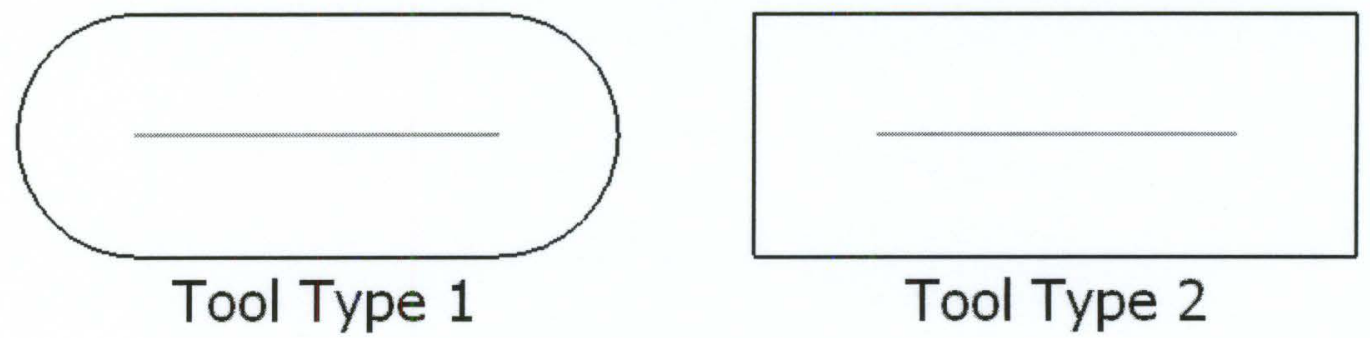

Figure 4.15: Centre Lines of Tool Type 1 and 2

In order for a line on the profile offset to be a solution, it must be able to reach all linear regions of the offset either through point contact or line contact. Fig. 4.16 shows tool centre lines engaged in both types of contact.

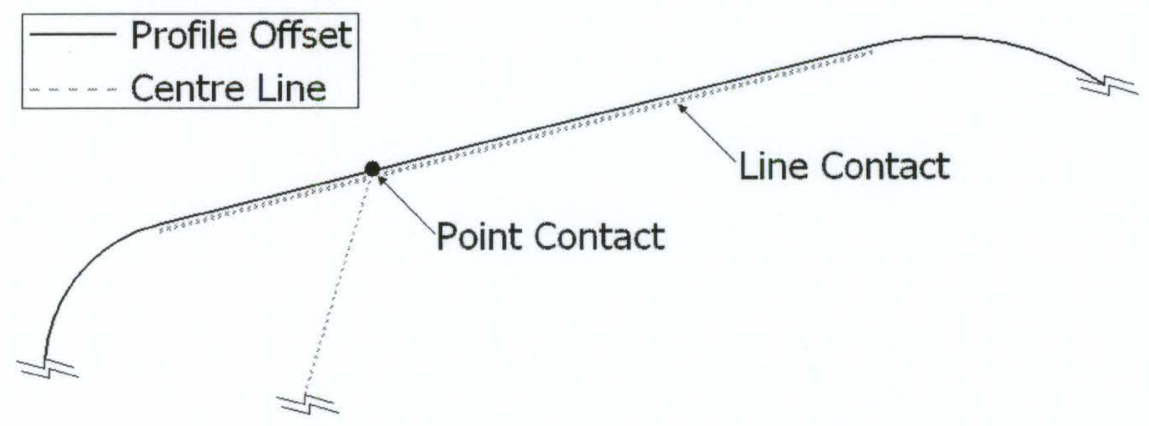

Figure 4.16: Tool Centre Lines Engaged in Point and Line Contact

The contact types presented in Fig. 4.16 form the basis for determining if the tool length defined by a line on the offset profile can reach all regions of the cavity profile. In addition to this, the interior angles of the profile offset must be considered.

Beginning with the longest line on the profile offset, the lines are searched in order of decreasing length. In most situations, the longest line will not be accessible to all regions of the profile offset. However, in situations when it is, the search may be stopped. When multiple lines exist on the profile offset, the search is terminated with the first length which can access all regions of the offset profile. 
When the centre line in consideration is tested for accessibility against offset lines which are longer in length, the centre line can easily access these lines by lying flat against them. If this is not the case, the centre line is positioned at the end of the offset curve against which it is being tested. The orientation of the centre line is such that it does not intersect the profile offset. Furthermore, the interior angle between the centre line and the offset curve is maximized.

If the tool centre line and the offset curve form an interior angle greater than or equal to $90^{\circ}$, a region which the centre line may sweep can be determined. This region is bounded by the centre line, the offset curve, and the line connecting the endpoints of both. When the boundary of this region does not intersect the profile offset, the tool centre line can access the entire curve through point contact. Fig. 4.17 shows a region of this type. It should be noted that this test must be performed from both ends of the offset curve.

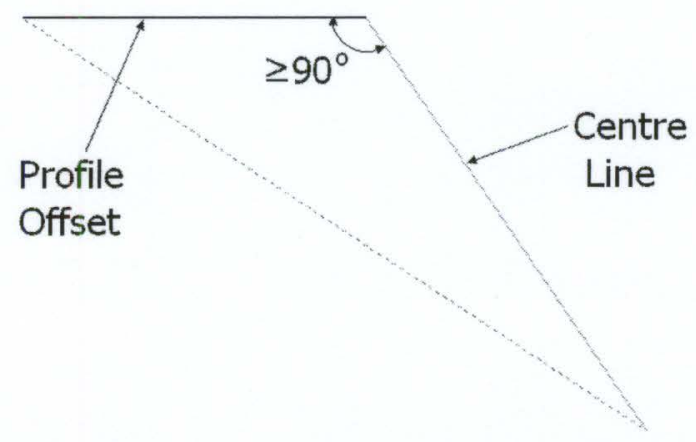

Figure 4.17: Bounded Region - Interior Angle $\geq 90^{\circ}$

If the tool centre line and the offset curve form an interior angle less than $90^{\circ}$, two situations may occur. The first is when the interior angle between the centre line and the offset curve is greater than or equal to $90^{\circ}$ at the opposite end of the curve. In this case, the approach described above will determine if the offset curve may be traced. It is applied to the end of the curve where the interior angle is greater than or equal to $90^{\circ}$. 
When both ends of the offset curve form an interior angle less than $90^{\circ}$, there must be a point on the offset curve where the centre line is oriented $90^{\circ}$ to the offset curve. The endpoint of the centre line at this location is connected to the endpoints of the centre lines at the ends of the offset curve. This is the region in which the tool may be swept. If this region does not intersect the profile offset, the tool may access the entire offset curve by point contact. Fig. 4.18 shows this case.

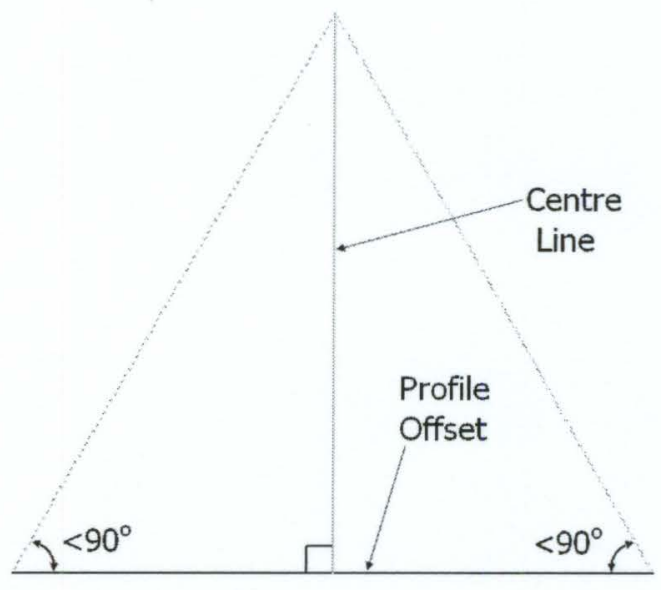

Figure 4.18: Bounded Region - Interior Angle $<90^{\circ}$

If there does not exist a location along the offset curve where the centre line is normal to it, the entire curve may not be fully accessed, unless the offset curve is a line shorter in length than the centre line.

When this method returns no result, the MIC is used to define the tool length in concave regions of the cavity. However, when it does, the tool length defined by this method is compared to that defined by MIC and the larger value is chosen to represent the maximum allowable tool length in concave sections.

Once the tool length has been defined for the concave regions of the cavity, it must be determined for the convex regions. This method depends on the geometry of the convex region. When the convex region is described by a tangent continuous curve, tangent lines constructed along the offset curve are used to calculate the length 
of the centre line. If the convexity occurs between tangent discontinuous curves, the centre line length is defined by a line perpendicular to the angular bisector of the curves. These cases are illustrated in Fig. 4.19.
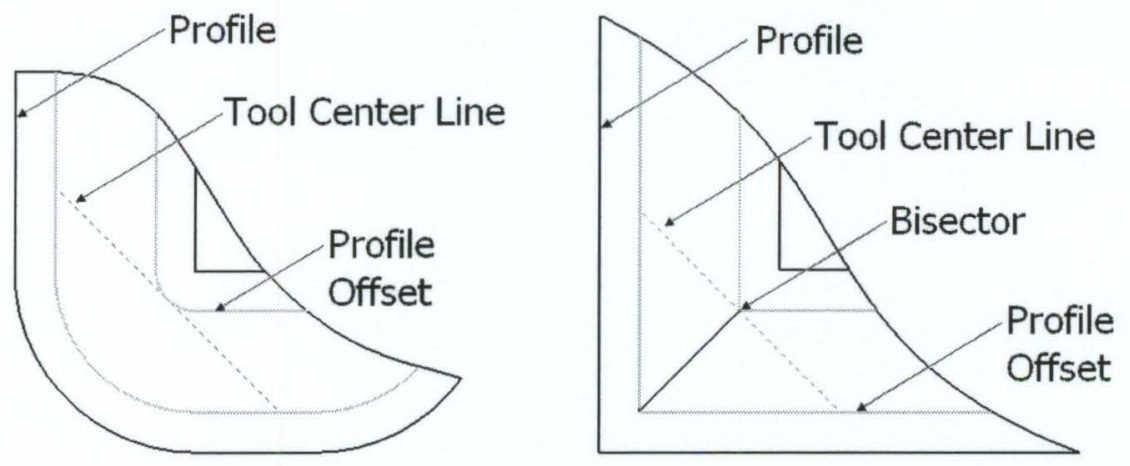

Figure 4.19: Tool Length Defined by Convex Regions on the Profile

For the tangent continuous case, tangents are constructed incrementally as the parameter defining the curve $(u)$ is incrementally increased from $u=0$ to $u=1$. At each step the tangents are constructed in both directions such that they intersect the profile offset. The length of the tangents on each side is expressed as a function of the parameter, $u$. Furthermore, the sum of the tangents on both the left and right hand side of the point is stored as a function of $u$. When $u=1$, the sums of the tangent lengths are searched for a minimum. The result of this search defines the centre line of the longest tool which can fit in the convex region. This test must be performed on all appropriate convex regions on the profile offset.

In the tangent discontinuous case, an angular bisector is constructed between the two curves which combine to form the convexity. A line is constructed which is perpendicular to the bisector and it is extended such that it intersects the profile offset. The length of that line is the length of the corresponding centre line for that section. This test must also be conducted for all appropriate convex regions of the profile offset. 
Once all of the tests have been performed for both concave and convex regions of the profile, the tool length may be determined. However, the diameter of the MIC must be reduced by the amount of the tool width for comparison since the other lengths represent the centre line of the tool. The reduced diameter of the MIC may then be compared with the centre line length calculated based on lines contained in the profile offset. The maximum of these values defines the centre line length for concave regions of the profile offset. This value must then be compared to the centre line length calculated for convex regions. The minimum of these values defines the length of the centre line. Once the length of the centre line has been determined, the tool length may be determined according to the tool type.

\subsubsection{Tool Length Calculation - Profile Type 1}

Profiles of type 1 are machined by a tool of type 1. These tools have rounded ends which can be represented by circles. When these circles lie on the profile offset, they are tangent to the profile regardless of their orientation. Since the tool centre line connects the centres of the circles defining the rounded ends of the tool, the tool length may be determined simply by adding the tool width to the length of the centre line. This is expressed mathematically in the following equation where $L$ is the tool length, $l_{c}$ is the length of the centre line and $w$ is the tool width.

$$
L=l_{c}+w
$$

The above equation applies to all methods of tool length determination for profiles of type 1 . When the profile is of type 2 , the computation required is slightly more involved. 


\subsubsection{Tool Length Calculation - Profile Type 2}

Due to the geometry of the tools used in the machining of type 2 profiles, the tool length may not be determined simply by adding the width of the tool to the length of the centre line. In this case, the tool must be inscribed in a circle whose diameter is equal to the sum of the centre line length and the tool width. The mathematical expression for determining the tool length, given the length of the centre line, is shown below.

$$
L=\sqrt{\rho^{2}-w^{2}}
$$

In the above equation, $L$ is the tool length, $w$ is the tool width and $\rho=l_{c}+w$. These variables are illustrated in Fig. 4.20. Also shown is the triangle from which the solution is obtained.

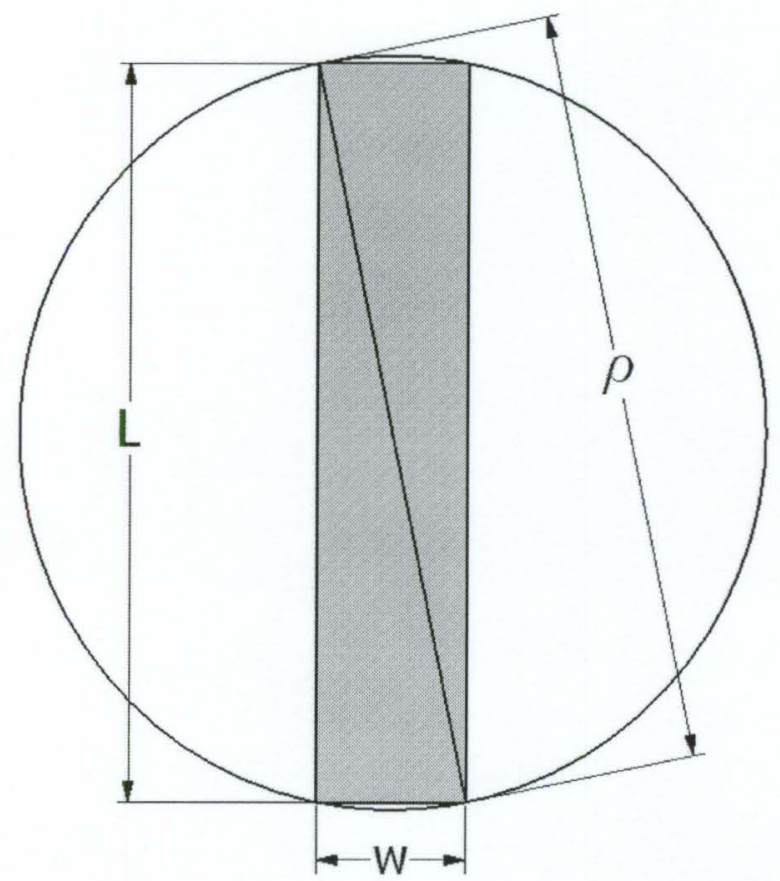

Figure 4.20: Variables in Eqn. 4.2 


\subsubsection{Tool Length Calculation - Profile Type 3}

Since the tools used to machine profiles of type 3 have one round end and one square end a combination of Eqns. 4.1 and 4.2 is used to determine the tool length. The length may be determined by adding half the value returned by Eqn. 4.1 and half of the value returned by Eqn. 4.2. Therefore, the equation for determining tool length is as follows.

$$
L=\frac{\sqrt{\rho^{2}-w^{2}}+\rho}{2}
$$

In this equation, $L$ is the tool length, $w$ is the tool width and $\rho=l_{c}+w$. Fig. 4.21 shows the division of a type 3 tool into its square half and round half thereby validating Eqn. 4.3.

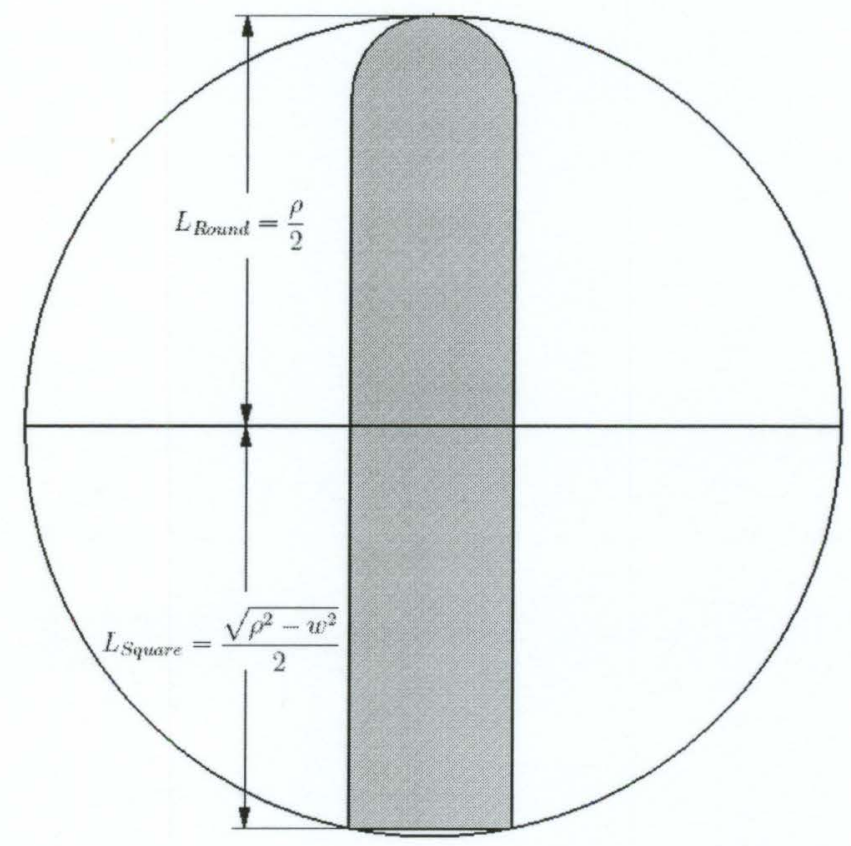

Figure 4.21: Length - Round and Square End of Tool Type 3

It should be noted that when a tool of type 2 is used to machine a cavity whose profile is of type 3, the tool length is determined as stated in $\S 4.5 .2$. 


\subsubsection{Tool Length Calculation - General Algorithm}

The general algorithm for tool length calculation is presented in Fig. 4.22.

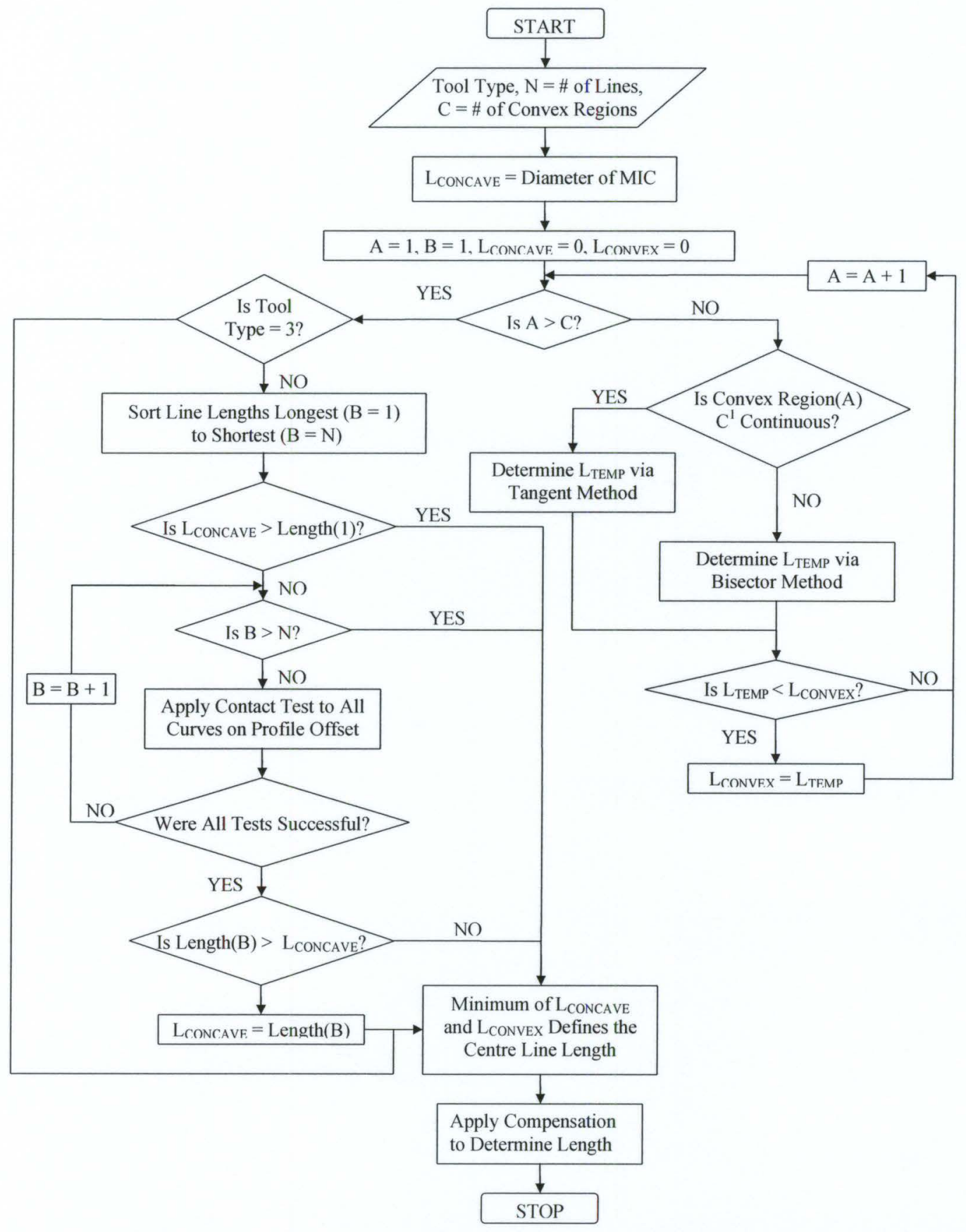

Figure 4.22: Tool Length Algorithm 
In Fig. 4.22, length is initially determined by the diameter of the MIC. Following this, the length is calculated at convex regions of the profile. If the tool is of type 3 , no further tests are required since the tool cannot be longer than the length defined by the MIC. However, when the profile contains concave region with lines whose lengths are larger than the diameter of the MIC, these lines must be considered in defining the tool length. When lengths for concave and convex regions of the profile have been determined, the largest concave length is compared to the smallest convex length. The smaller of these values defines the length of the centre line. Compensation is applied by the appropriate equation, Eqn. 4.1 to 4.3.

Once the tool width and the corresponding permissible length have been determined, an appropriately sized tool may be selected. A layer-by-layer tool path may then be generated according to the dimensions of the tool selected. 


\section{Chapter 5}

\section{Tool Path Planning}

Tool path planning strategies have been sufficiently developed for the machining of 2D pockets with cylindrical tools and are summarized in [44]. In this work, Hatna et. al. [44] state that the primary objective in tool path planning is to cover the entire machinable area without damaging the finished surface. Though little research has been done in tool path planning with non-cylindrical tools, the primary objective remains unchanged.

Tang et. al. [45] studied the area swept by a polygon undergoing only translation. In this work, the generator polygon (generatrix) was constrained to and driven along another polygon, the directrix. The swept area is defined by the union of the instantaneous areas occupied by the polygon traveling around the directrix. In some cases, it was shown that the generator polygon could not completely trace the directrix. This was referred to as undercut and instances in which it would occur were discussed.

Sambandan et. al. [46] studied the planar sweeping of polygons. In this work, they developed a technique for computing the area swept by a polygon undergoing both translation and rotation. The swept area was defined as the union of the areas 
covered by the polygons at different times. Sambandan et. al. [46] developed a set of criteria to determine which points and lines on the polygon lie on the profile of the swept area at time $t$. The resulting profile is then trimmed and the only the boundary remains. This approach was further developed by Ling and Chase [47] for applications in planar mechanisms.

Tool paths in this work are generated for machining on a 4-axis EDM with noncylindrical tools. The position of the tool in space is defined by a three-dimensional right-handed coordinate system, while the orientation of the tool is defined by an angular coordinate in the xy-plane centred at the z-axis. Fig. 5.1 shows the machine tool coordinate system.

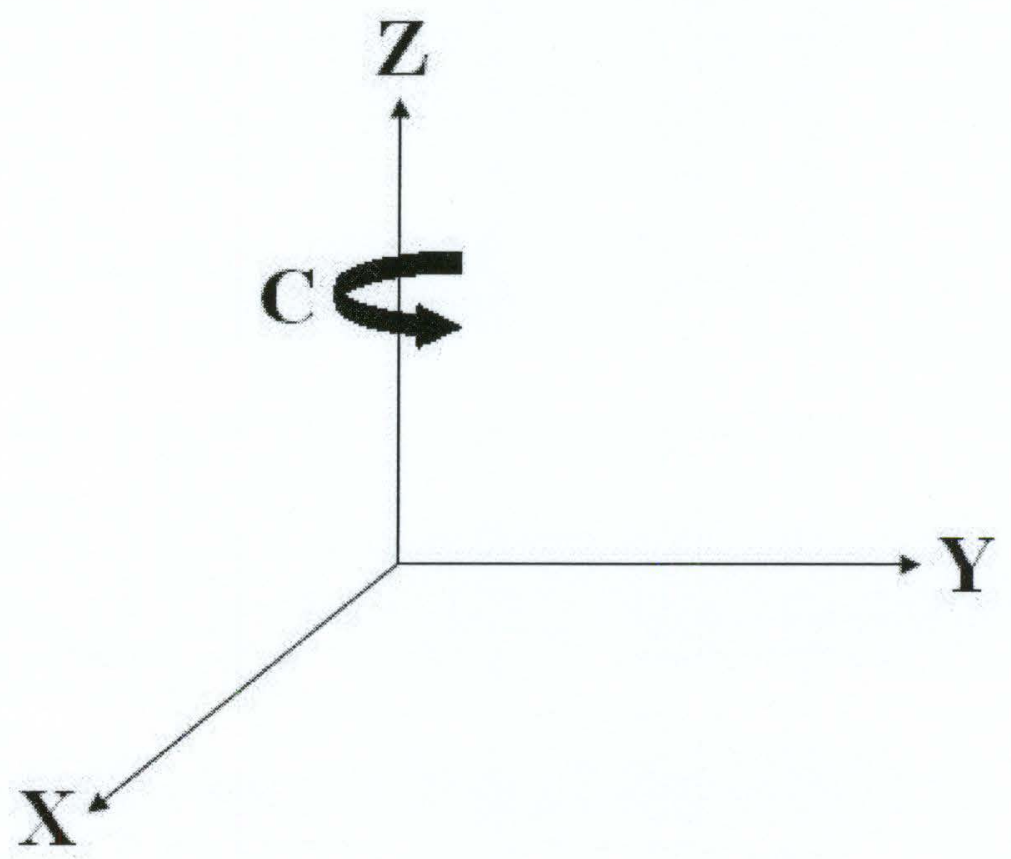

Figure 5.1: Coordinate System for a 4-Axis EDM Machine Tool

The coordinate system presented in Fig. 5.1 allows for the following types of relative motion between the tool and workpiece. 
1. Translation in $\mathrm{X}, \mathrm{Y}, \mathrm{Z}$

2. Rotation about the z-axis

3. Translation in $\mathrm{X}, \mathrm{Y}, \mathrm{Z}$ with simultaneous rotation about the $\mathrm{Z}$-axis

Using these motions, two-dimensional tool paths are generated in the xy-plane in which machining occurs along the frontal face of the tool. To maximize machining time, the tool is not to be retracted from the workpiece during machining. To achieve the desired depth, the two-dimensional tool paths are executed layer-by-layer in the negative z-direction.

Before a tool path can be generated, the number of passes required to completely machine the $2 \mathrm{D}$ cavity profile must be determined. Since the algorithm is not dependent on profile type, it will be presented generally for all profile types.

To determine the number of passes required to machine a $2 \mathrm{D}$ profile, the outermost boundary of the profile must be offset by the tool length. It should be noted that islands are not offset since they are traced by the trailing end of the tool. Upon offsetting the outermost boundary profile by the tool length, closed loops are formed. Any loops which are not entirely contained in the profile are discarded. The remaining loops are used to determine whether or not multiple passes are required.

If a closed loop is contained in the profile and is composed entirely of curves whose direction of offset point toward the interior of the loop, multiple passes are required to machine the region bounded by the loop. The reason for this is that when the tool tip is tracing the profile, it attempts to remain normal to it. Any regions that the tool cannot sweep while it is normal to the profile may be determined by offsetting the profile by the tool length. This procedure is applied to the remaining closed loops to determine whether a further pass is required. Fig. 5.2 shows when a tool (a) can and (b) cannot machine the cavity in a single pass. 

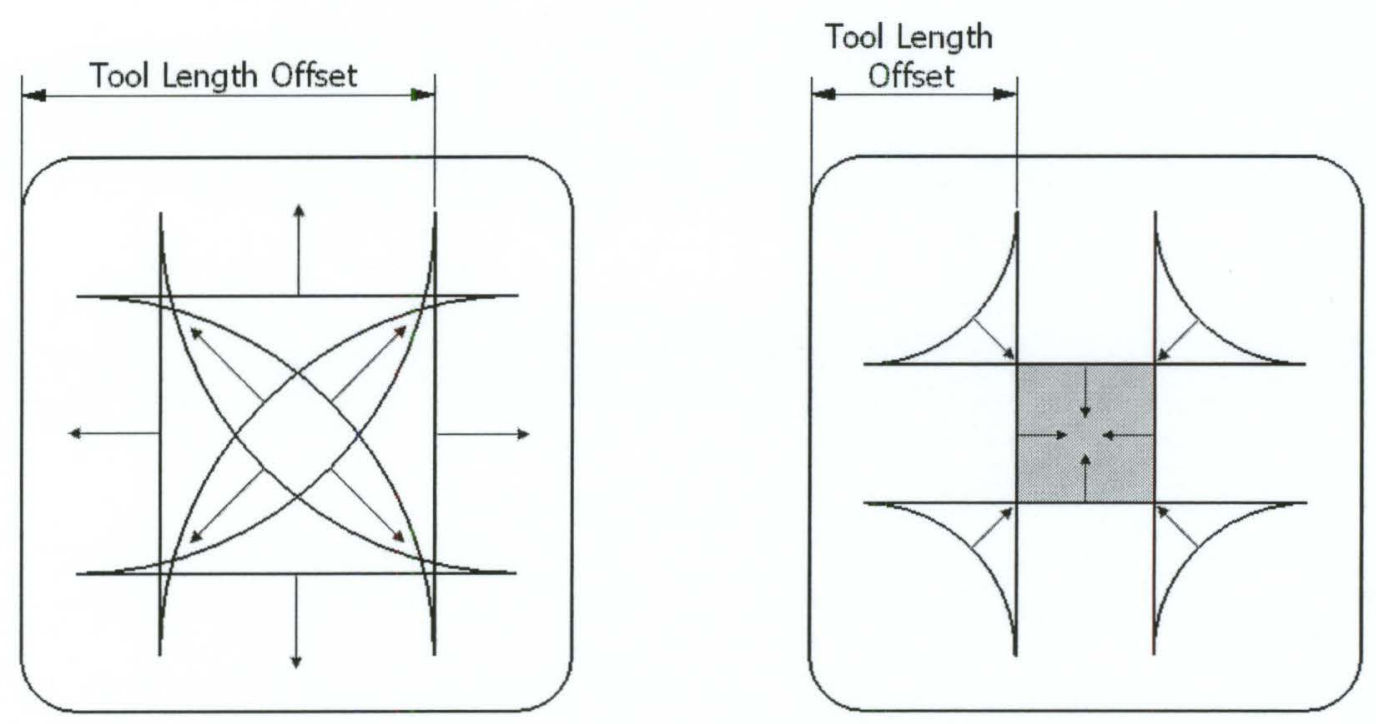

Figure 5.2: (a) Single Pass Required and (b) Multiple Passes Required

In (a), there are no closed loops entirely composed of curves whose direction of offset points towards the loops interior. This means that the length of the selected tool is sufficient to machine the entire cavity in one pass. In (b), there is a region composed entirely of curves whose offset directions point to the interior of the loop. That region is shaded in gray. Though the cavities are the same, the reduction in tool length is sufficient enough to require multiple passes.

Once the number of passes required to machine the profile has been determined, the critical points may be determined.

Critical points are points along the tool path at which the tool may become or may not remain normal to the profile. Like the number of passes, critical points are also determined by offsetting the profile by the tool length. Any intersection points between the tool length offset and the profile result in critical points. The point on the original profile which corresponds to the point of intersection on the tool length offset is stored and used to compute the tool orientation during tool path planning. This concept is shown in Fig. 5.3. 


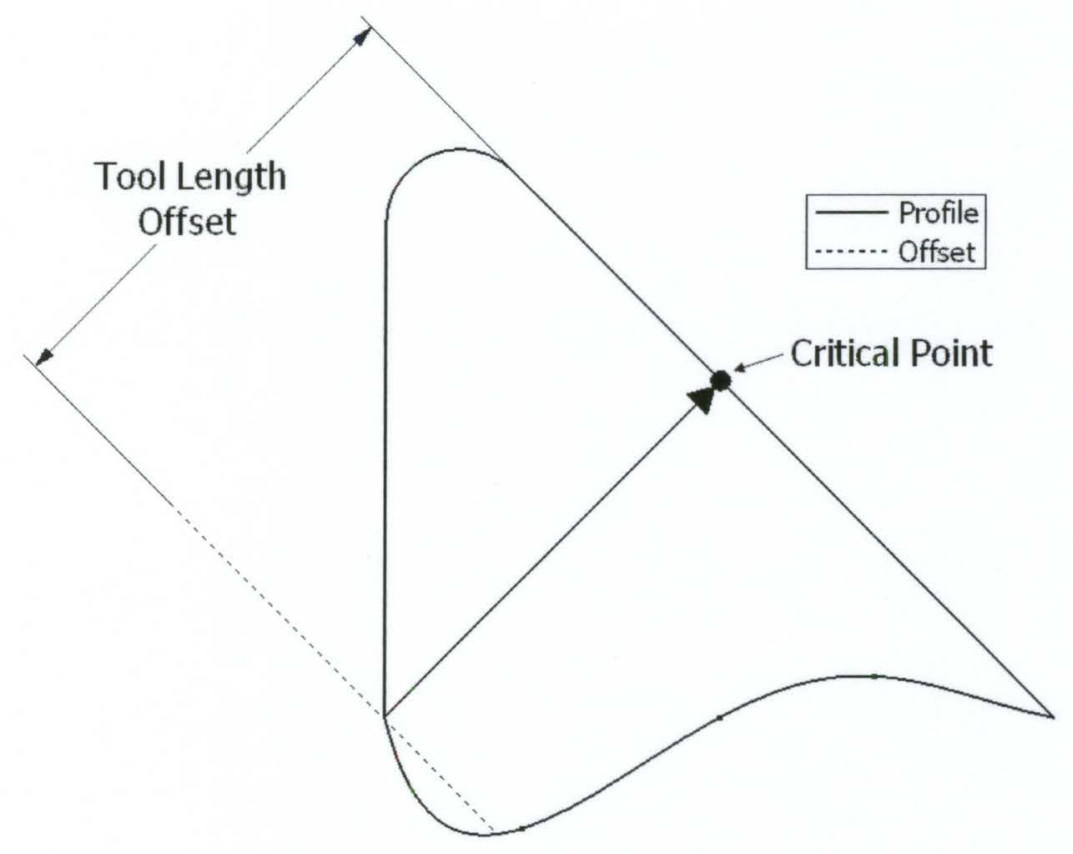

Figure 5.3: Determination of Critical Points

Upon calculating the number of passes and the location of the critical points on the profile, the tool paths may be computed. However, due to differences in the tool path planning strategies used for each of the profile types, the critical points must be further manipulated. Therefore, the tool path planning algorithms specific to profile type are presented.

\subsection{Tool Path Planning - Profile Type 1}

Profiles of type 1 are free of sharp corners and resemble profiles which are typically machined using a cylindrical tool. Therefore, the tool path planning strategy used for machining type 1 profiles is derived from the offset strategy used in end milling, where the tool is driven along successive offset curves.

In this approach, the cavity profile and any regions requiring multiple passes must first be offset by half of the tool width. The resulting curves are termed offset 
curves. Any offset curves belonging to regions requiring multiple passes must then be attached to the profile offset by a line. The centre of the arc, which defines the rounded end of the tool, is constrained to the profile offset ensuring that the entire boundary of the profile is machined. Fig. 5.4 shows a tool constrained to the profile offset and presents some of the terminology used in this section.

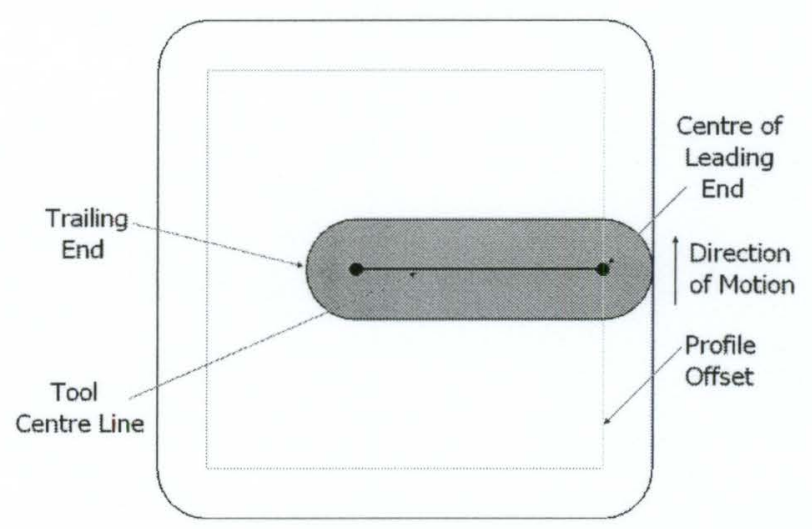

Figure 5.4: Tool Constrained to the Offset Curve

To ensure that the interior of the profile is fully machined, the orientation of the tool must be determined. Therefore, the critical points determined earlier must be recalled. The critical points which lie on the profile are projected onto the offset curves to which the tool is constrained.

Calculation of the tool orientation is simplified by using the tools centre line. The centre line of the tool is the line that connects the centres of the arcs which represent the tools tips. If the trailing end of the centre line intersects the offset profile, the tool will overcut the profile.

To avoid the occurrence of overcut, the change in orientation between end points of the offset curves must be determined. If the tool is normal to the profile at the start of the offset curve along which it is being driven, it will remain so until a critical point is reached. When a critical point is reached, the tools orientation 
must be changed such that no portion of the its centre line crosses the profile offset. Incremental solutions for the tool orientation are determined such that the trailing end of the tool remains behind the leading end, with respect to the direction of motion. However, this is not always possible, meaning that in some situations, the opposite result will occur.

When the tool is not normal to the profile at the start of the curve, the same approach is applied except the tool regains its normal orientation as it approaches the critical point. Again, the tool orientation is solved as the tool is driven along the curve such that the centre line of the tool does not intersect the offset profile. Upon being driven past all of the critical points on the offset curve, the tool orientation must be known at the endpoint of the curve so the tool will be in the proper orientation for the start of the next curve.

Fig. 5.5 shows examples of varying tool orientation when the tool begins normal to the profile and when it does not. The profile is shown along with the offset curve. Tool motion is in the direction of the arrow and the numbers 1 and 2 indicate the start and end positions of the tool, respectively. Critical points are indicated by dots.
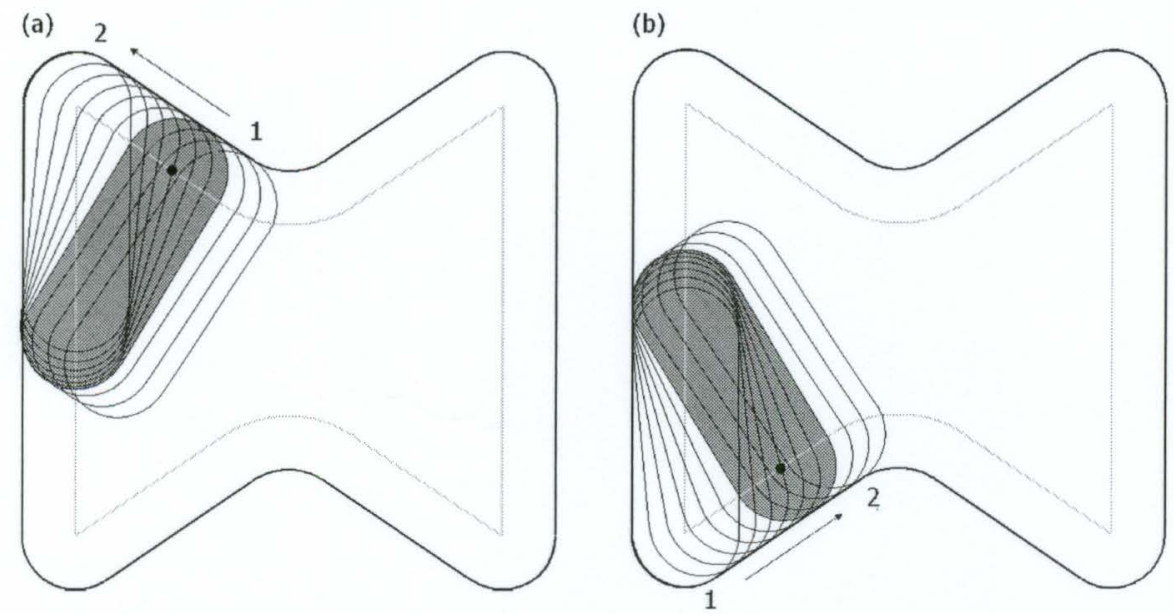

Figure 5.5: (a) Tool Normal and (b) Not Normal to Profile at Starting Point 
If an offset curve contains no critical points and the tool is normal to the profile at its starting point, the tool can maintain its orientation (with respect to the profile) along the entire curve. However, when the offset curve contains no critical points and the tool is not normal to the profile at the start of the curve, the orientation must be solved incrementally along the entire curve.

When the tool returns to its start position satisfying the conditions for multiple passes and critical points, a cutter location (CL) file can be generated. The CL file stores the position $(x, y)$ and orientation $(c)$ of the tool. A post-processor was developed in MATLAB which converts the CL file into G-code. The post-processor also adds the z-coordinate and generates a to-and-fro type tool path in which each consecutive layer is machined in the opposite direction.

\subsection{Tool Path Planning - Profile Type 2}

Profiles of type 2 contain sharp corners and may not be machined with a cylindrical tool. Therefore, a different approach to tool path planning must be considered.

The tool used to machine profiles of type 2 contains four sharp corners. It is shown in Fig. 5.6 along with some terminology used in this section. In order for this tool type to completely machine the profile, at least one of the corners must lie on the profile at all times. Therefore, the tool is constrained to the cavity profile as opposed to its offset curve.

Before the tool path may be generated, regions requiring multiple passes must be connected to the profile boundary by a line. Once all regions requiring multiple passes have been connected to the profile boundary, the tool path may be computed. 


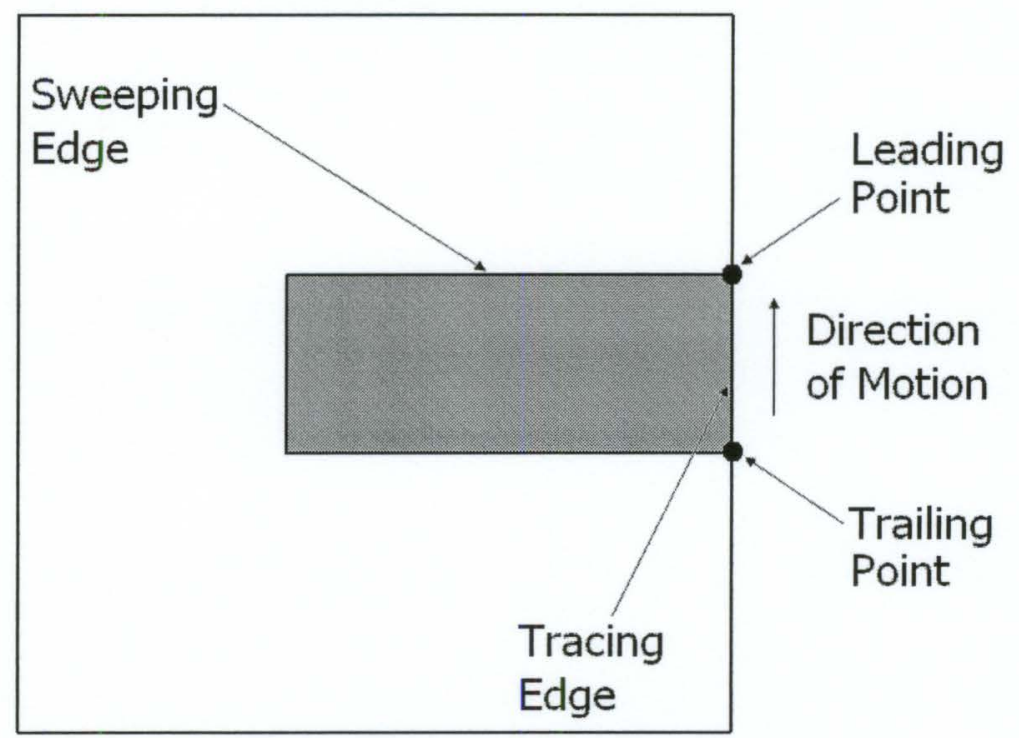

Figure 5.6: Tool Constrained to the Profile

The starting position of the tool is selected such that the tool is normal to the profile. This ensures that the tracing edge of the tool, defined by the tool width, will fully be in contact with the profile. The endpoints of the tracing edge are conditionally constrained to the profile. At least one of these points must lie on the profile during machining of the cavity.

From the starting position, the tracing edge is advanced along the profile, in the counter-clockwise direction, until the leading point reaches a critical point. When the tool reaches a critical point while it is normal to the profile, it will lose its normal orientation. The corresponding change in orientation causes the leading point of the tool to be removed from the profile meaning that only the trailing tip remains on it. Fig. 5.7 shows a case when this occurs. In (a), the tool orientation at the critical point is shaded. A tool orientation in which the leading point is removed from the profile is shown in (b). 

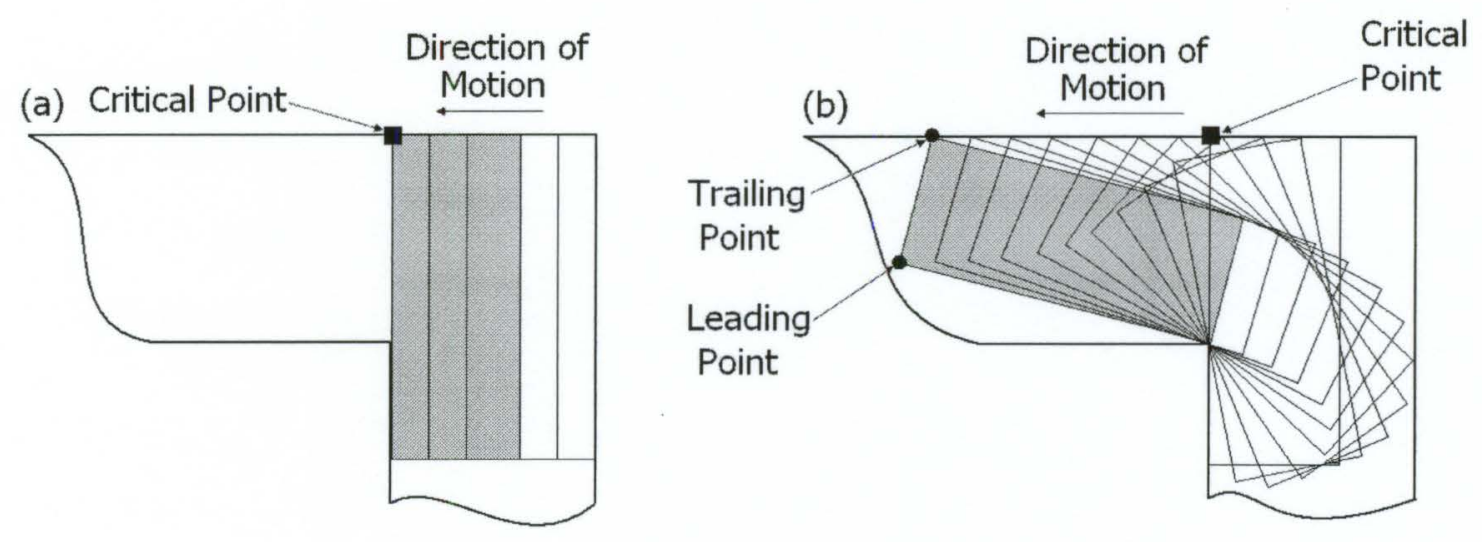

Figure 5.7: Profile Type 2 - Critical Points

Computation of the tool orientation is dependent on the trailing point and the sweeping edge of the tool. The trailing point must remain on the profile and the sweeping edge must not intersect the boundary profile. Representing the sweeping edge as a line allows for the tool orientation to be computed based on intersections. This computation is performed incrementally. If the tool reaches another critical point on the same curve, it can become normal to the profile again. When the curve contains no further critical points, the leading point cannot be re-constrained to the profile. In this case, the trailing point will still lie on the profile. Therefore, it must trace the remainder of the curve as the leading point advances to the next curve meaning both the leading and trailing point are constrained to the profile. This concept is shown in Fig. 5.8. The initial tool configuration is shaded.

In Fig. 5.8, the trailing point traces (a) - (b) on the current curve, while the leading point traces (b) - (c) on the following curve. Once the trailing point has reached the (b), the leading point continues tracing the profile as it travels along the next curve. 


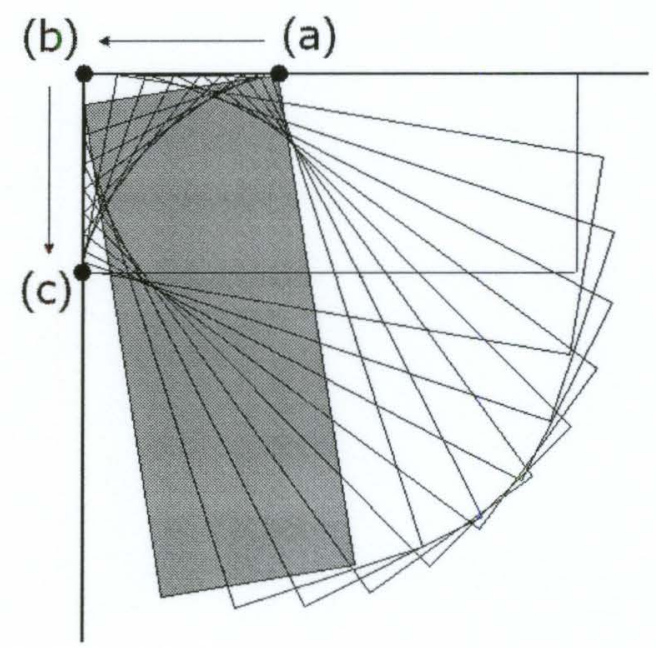

Figure 5.8: Trailing Point Tracing the End Portion of a Curve

The tool path is complete when the tool has traced the entire profile, returned to its starting position and satisfied the conditions of multiple passes and critical points. When this occurs, a CL file can be generated based on the calculated tool positions/orientations. The CL file is converted to G-code by the post processor so that the tool path may be implemented on a 4-axis CNC EDM machine tool.

\subsection{Tool Path Planning - Profile Type 3}

Tool path planning for the machining of type 3 profiles with a type 3 tool is presented in this section. It should be noted that profiles of type 3 may be machined with tool type 2. However, in most cases a tool of type 2 is much smaller than the equivalent type 3 tool. In the case where a tool of type 2 is larger in size, the tool path planning strategy presented in $\S 5.2$ is applied.

The tool used to machine most type 3 profiles has both a rounded end and a square end allowing it to trace both sharp corners and smooth curves without compromising its size. Since the tool must be able to fully rotate within the profile, it 
may not have a length longer than that defined by the MIC. Where the MIC touches the profile boundary, is where the tool path begins. This allows the tool to rotate and switch its tracing end from rounded to square and vice versa.

Since the tool contains both rounded and sharp ends, two 2D tool paths must be generated. The first of these paths is determined by the round end of the tool. To develop a tool path for this end of the tool, the cavity must be modified so that it is a profile of type 1. This representation is achieved by joining any pairs of concave curves which are $\mathrm{G}^{0}$ continuous with a bi-tangent circular arc of radius equal to half the tool width. In other words, fillets are constructed between the curves. The result is a profile which can be machined by the rounded end of the tool. Fig. 5.9 shows a type 3 profile and its corresponding type 1 profile representation.

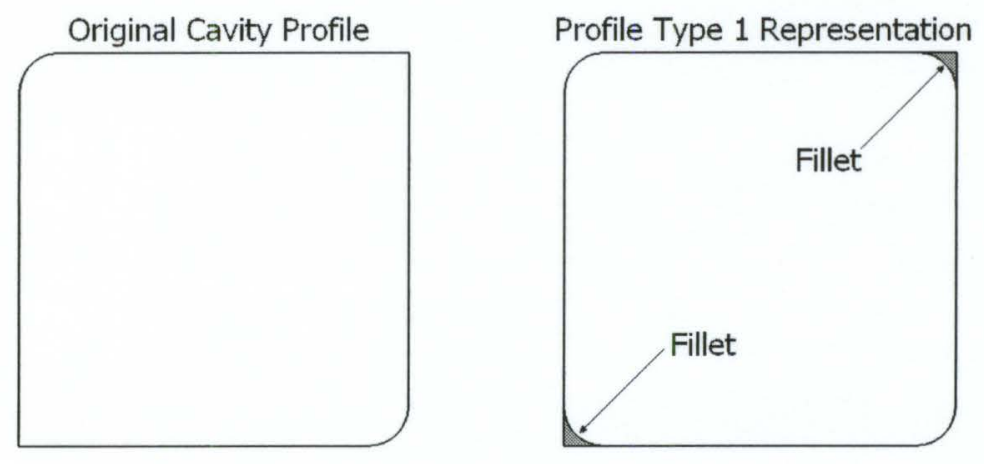

Figure 5.9: Profile Type 1 Representation of a Type 3 Profile

Once the tangent continuous representation of the profile has been determined, it must be offset by half of the tool width. The centre defining the round end of the tool is then constrained to the profile offset. As it is driven along the offset profile, it follows the strategy outlined in the tool path planning for cavities of profile type 1 with some modifications. Since the opposite end of the tool is square, the tool orientation must be calculated such that the sweeping edge does not intersect the profile boundary. 
As the tool returns to its starting position, meeting the conditions of multiple passes and critical points, the tool must be rotated $180^{\circ}$ such that its square end lies on the cavity profile. A CL file may then be generated which includes the rotation of the tool. The final position of this layer corresponds to the starting position of the next layer.

Using the original representation of the cavity profile, the tool is driven along the profile from the starting position determined in the previous layer. As the tool travels along the profile, it follows the methods outlined in the tool path planning for a profile of type 2. However, since the opposite end of the tool is rounded and the profile offset is known, the tool orientation may be determined using the tools centreline. When the tool orientation is being changed and the square end of the tool is tracing the profile, the centre of the tools rounded end may not intersect the profile offset. Furthermore, the trailing point on the tracing edge of the tool must remain constrained to the profile boundary during changes in tool orientation.

Some non-linear curves on the boundary profile may not be reached by the square end of the tool. However, regions which cannot be reached by the square end of the tool are non-linear and thus are machined by the rounded end of the tool. As the tool traces these curves, a position exists where the leading point and the trailing point cannot advance in the counter-clockwise direction. At this point, the tool is stuck and cannot machine the entire curve. Since this region is accessible to the rounded end of the tool, the leading point on the square end continues along the profile boundary. Fig. 5.10 shows a situation where only the round end of the tool can completely machine the non-linear region of the profile. The square end of the tool is shown in a position where it cannot trace the curve any further. 

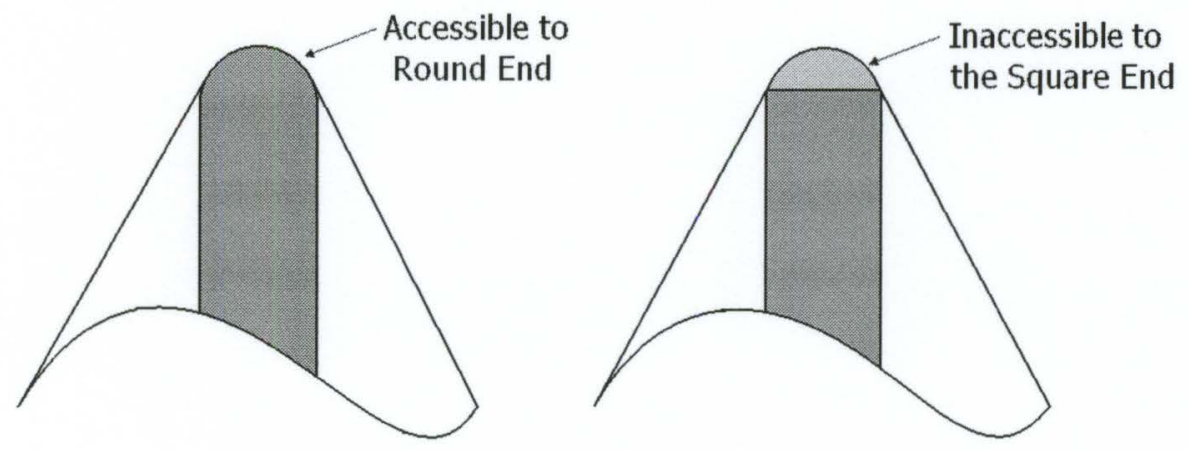

Figure 5.10: Profile Curve Traced by Only the Round End of the Tool

When the square end of the tool reaches the position shown in Fig. 5.10, it continues to travel along the profile in the counter-clockwise direction leaving the curve to be traced by the rounded end of the tool.

Once the tool has reached its starting position and has satisfied the conditions of multiple passes and critical points, it is again rotated $180^{\circ}$ such that the centre of the tools round end lies on the profile offset. A second CL file can now be generated.

The post processor uses both CL files to generate the G-code required to implement the tool path on a 4-axis EDM. Alternating between the two CL files as the tool position decreases in the z-direction produces a layer-by-layer tool path in which each successive layer is machined by the opposite end of the tool. Therefore, the resulting tool path machines the entire cavity.

\subsection{Tool Path Planning - General Algorithm}

A general algorithm for tool path planning is presented in Fig. 5.11. The algorithm chooses the appropriate methodology based on the tool type selected and determines when to adjust the tool orientation. Once the tool path is complete a CL file is generated which is converted to G-code by a post processor. 


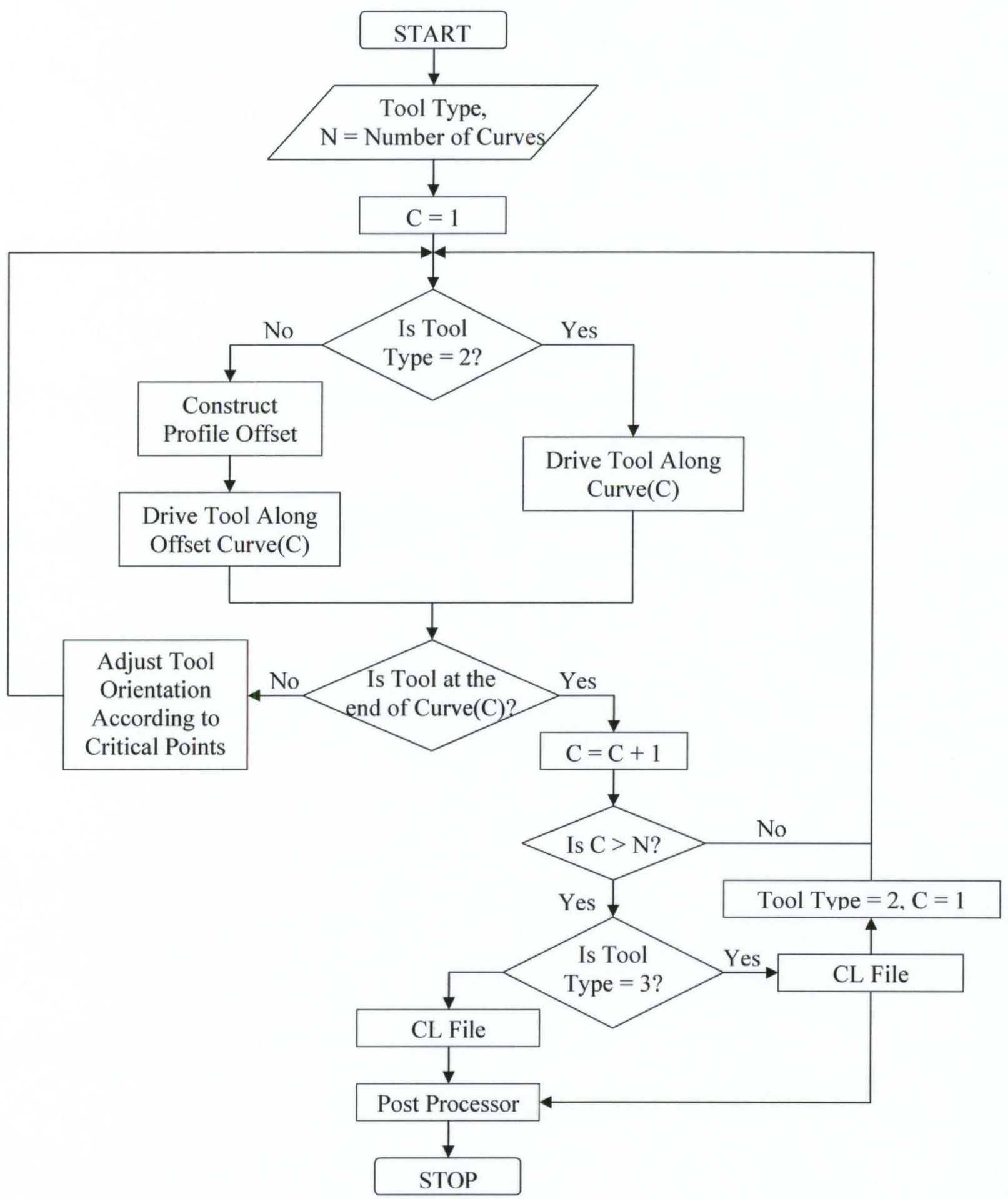

Figure 5.11: Tool Path Planning Algorithm 
Upon creating the tool path for a cavity and generating the corresponding Gcode, machining of the cavity may be realized on a 4-axis CNC EDM. In this work, two cavities were machined in which the tool type, tool size and tool path were calculated. These cavities are presented in the following section. 


\section{Chapter 6}

\section{Experiments \& Results}

In this chapter, the algorithms presented in this work are applied to the 4-axis rough machining of $2 \mathrm{D}$ cavities. For each cavity, the appropriate tool type and its size constraints were determined. A tool whose width and length are less than or equal to the respective constraints was chosen and the tool path was generated accordingly. Machining of the cavities was performed on a 4-axis EDM.

Mechanical milling was the process selected to manufacture the copper tools used for ED milling of the cavities. The cutting tool used during the milling of the copper tools was a 1 inch diameter high speed steel (HSS) end mill with four flutes. The machine tool used was a CNC milling machine. According to cutting tool and workpiece properties, the feed-rate and spindle speeds were $80 \mathrm{~mm} / \mathrm{min}$ and $300 \mathrm{RPM}$, respectively. A finishing pass was performed at a feed-rate of $30 \mathrm{~mm} / \mathrm{min}$.

During the machining of the tools, a System 3R table chuck was used to hold the tool. The table chuck is identical to the chuck which holds the tool on the EDM. However, the bottom surface has been ground allowing it to be used for off-machine truing of tools. This is important as it promotes a uniform gap during EDM. 


\subsection{Example 1 - Star Shaped Cavity}

To demonstrate the algorithms discussed in this work as they apply to cavities of profile type 1, a star shaped profile is presented. Fig. 6.1 shows a dimensioned view of the cavity profile in which dimensions of length are in $\mathrm{mm}$ and angular dimensions are in degrees. Since each prong of the star is identical, only one prong is dimensioned. The remaining dimensions may be determined by symmetry.

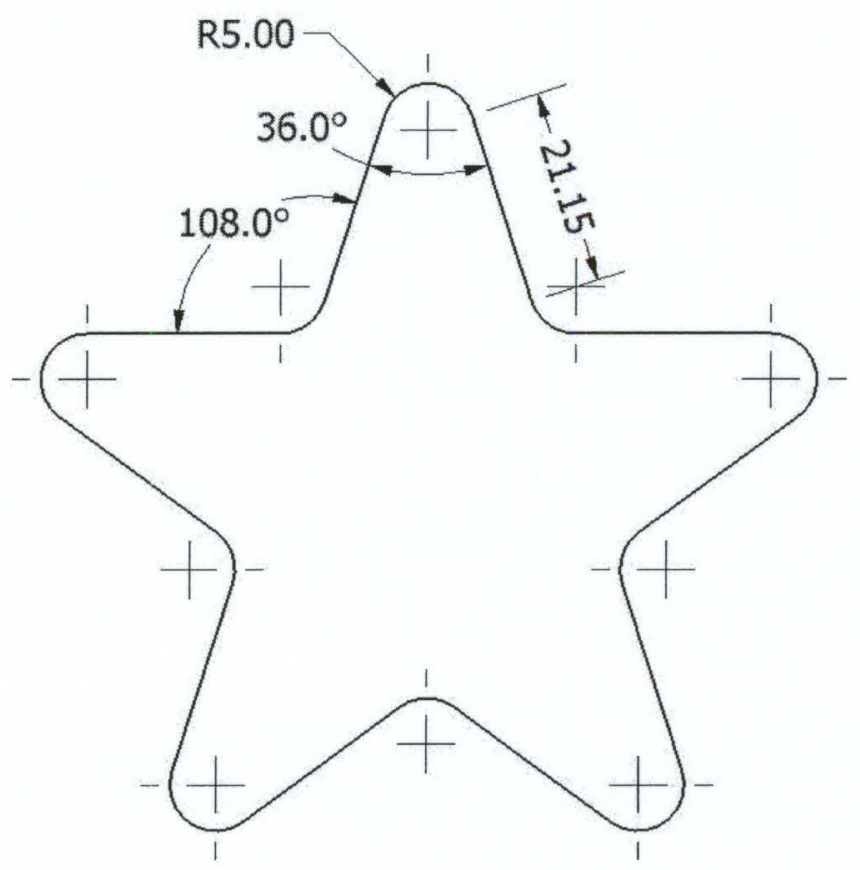

Figure 6.1: Star Shaped Cavity (Dimensions in $\mathrm{mm}$ )

In order to classify the profile type automatically, the profile must be represented by the lower order curves from which it is composed. Furthermore, the connectivity between pairs of curves must be known. This information allows for the algorithm presented in $\S 4.2$ to determine the profile type and thus the appropriate tool type. Fig. 6.2 shows the individual curves contained in the profile. The curves are numbered in a counter-clockwise fashion while their endpoints are represented by dots. Tangency between curves is indicated by a tangent continuity symbol. 


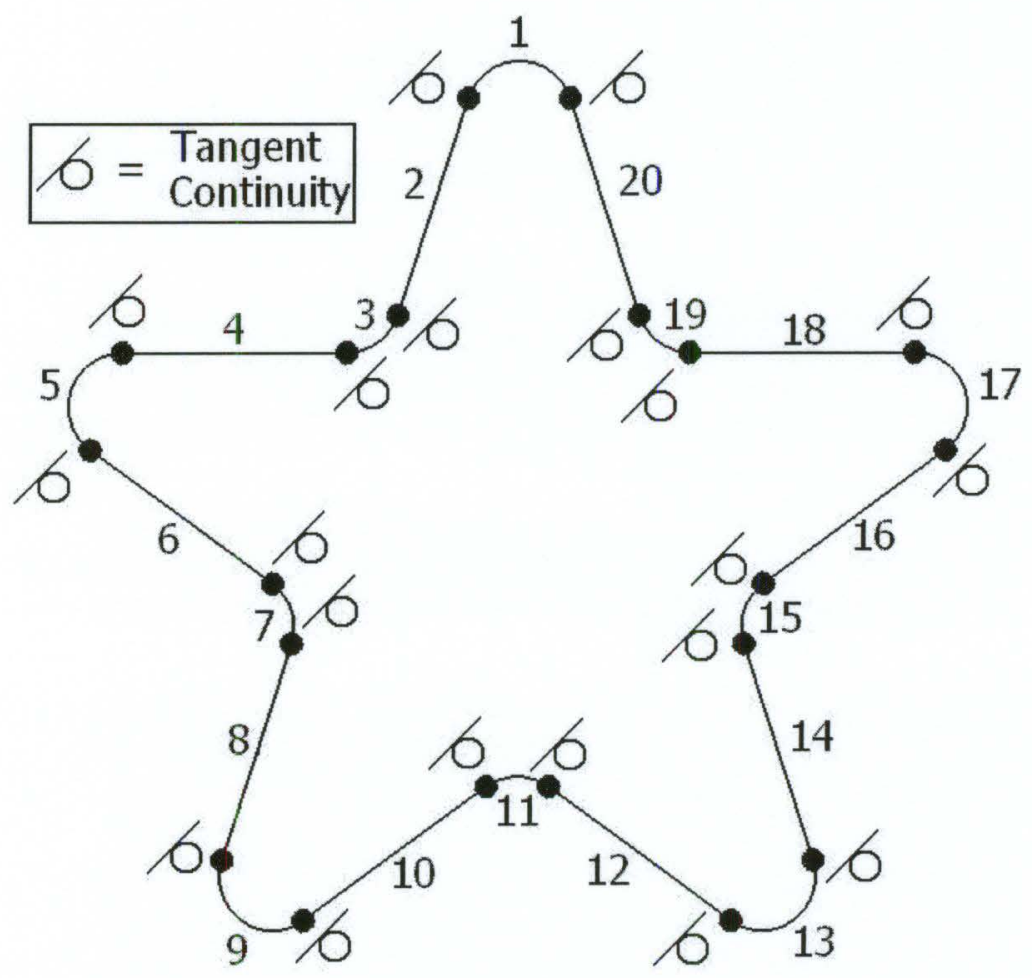

Figure 6.2: Curves Composing the Profile of the Star Shaped Profile

Applying the tool type selection algorithm to the profile representation shown in Fig. 6.2 indicates that the cavity profile is of type 1. All tests for tangent continuity between pairs of curves indicate that the entire profile is $\mathrm{G}^{1}$ continuous. The results of these tests are summarized in Table 6.1. Curve pairs which belong to concave regions are highlighted. By association, the algorithm suggests the use of tool type 1 for machining of this cavity.

\begin{tabular}{cc|cc|cc} 
Curve Pair & Tangent Continuous & Curve Pair & Tangent Continuous & Curve Pair & Tangent Continuous \\
\hline \hline $1-2$ & Yes & $8-9$ & Yes & $15-16$ & Yes \\
$2-3$ & Yes & $9-10$ & Yes & $16-17$ & Yes \\
$3-4$ & Yes & $10-11$ & Yes & $17-18$ & Yes \\
$4-5$ & Yes & $11-12$ & Yes & $18-19$ & Yes \\
$5-6$ & Yes & $12-13$ & Yes & $19-20$ & Yes \\
$6-7$ & Yes & $13-14$ & Yes & $20-1$ & Yes \\
$7-8$ & Yes & $14-15$ & Yes & &
\end{tabular}

Table 6.1: Star Shaped Cavity - Tangent Continuity Search Results 
Upon selection of the tool type, tool size calculations were performed. The first of these calculations was tool width. According to the algorithm presented in §4.4.4, if the tool is a type 1 tool, only the MAT is required. Searching the radius function of the MAT for a minimum will result in the maximum permissible tool width. The MA and the circles representing the minimum value of the radius function are shown in Fig. 6.3.

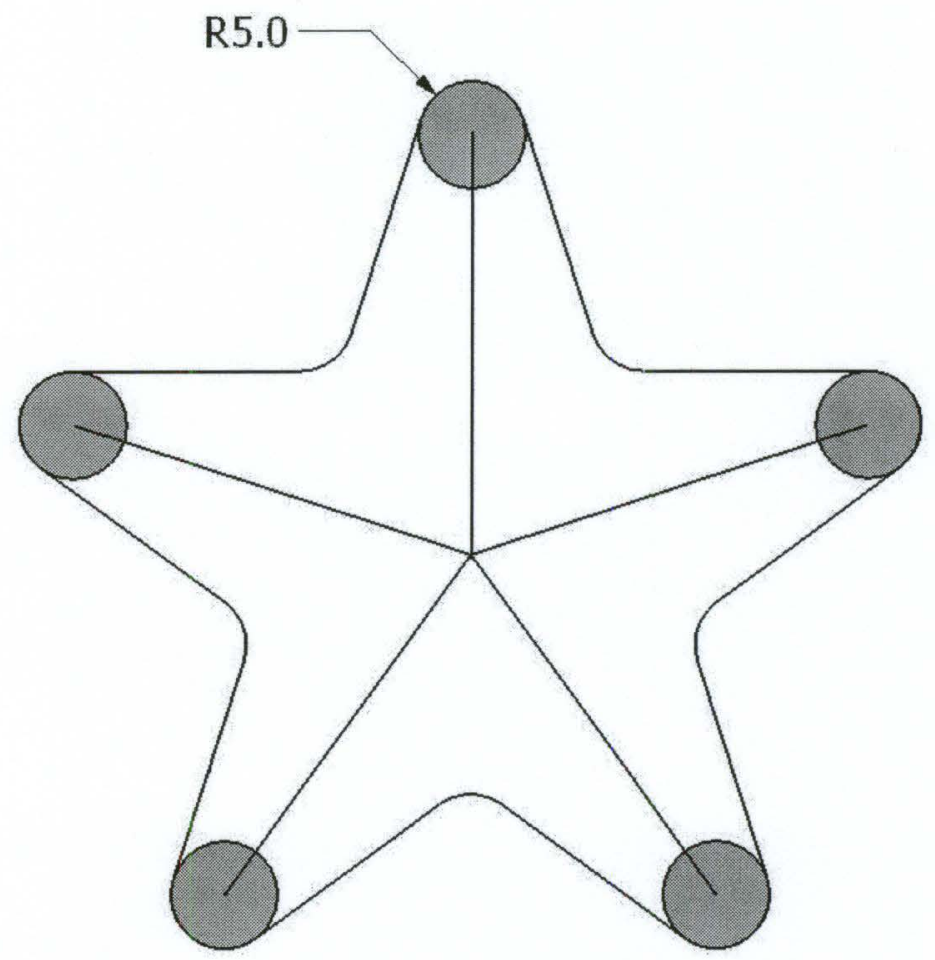

Figure 6.3: Star Shaped Cavity - Medial Axis

The circles representing the minimum value of the radius function were located at the tips of the star. This result was due to tapering. When the profile tapers, the radius function of the corresponding branch decreases in the direction of the taper. In this case, the minimum radii along such branches define the maximum permissible tool width. The corresponding diameter, $10 \mathrm{~mm}$, was chosen to define the tool width. 
Once the tool width was determined, the maximum permissible tool length was calculated. First, the radius of the MIC was determined. The resulting diameter and thus tool length was $44.6 \mathrm{~mm}$. Fig. 6.4 shows the MA, the MIC, and the corresponding tool.

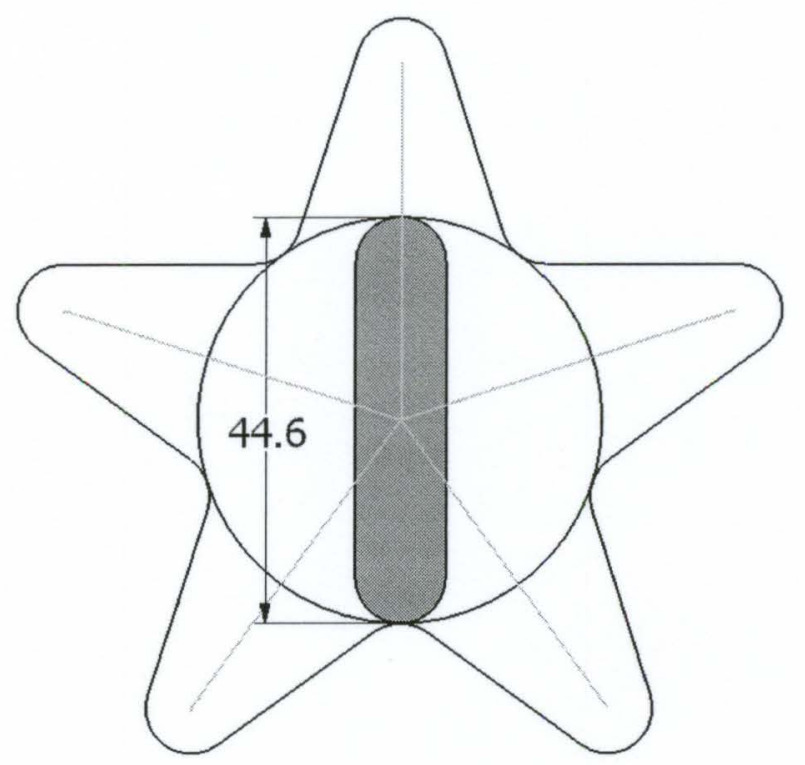

Figure 6.4: Star Shaped Cavity - MIC and Corresponding Tool Length

Since the profile contains five convex regions, the tool length at these regions was determined. To calculate the length of the centre line in the convex regions of the profile, the profile was offset by half of the tool width $(5 \mathrm{~mm})$. The offset is shown in Fig. 6.5. Also shown in the figure is one of the convex offset curves.

Tangent lines are constructed along these curves so that both ends of the line intersect the offset profile. The summation of the resulting lengths represents the centre line length at that point on the curve. As described in $\S 4.5$, the minimum of these values defines the maximum centre line length. The results of this search, at one of the convex regions, are shown in Fig. 6.6. Tool lengths on the left and right side of the tangent points are presented along with their summation. The minimum of the summation, which constrains the tool length, is also indicated. 


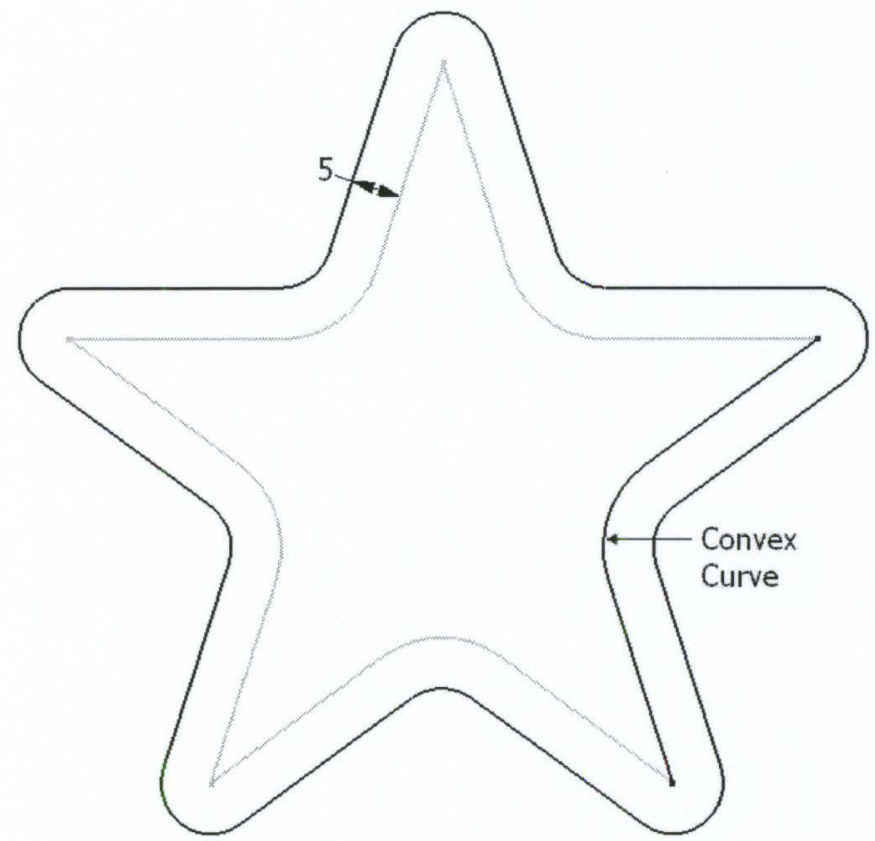

Figure 6.5: Star Shaped Cavity - Profile Offset by Half the Tool Width

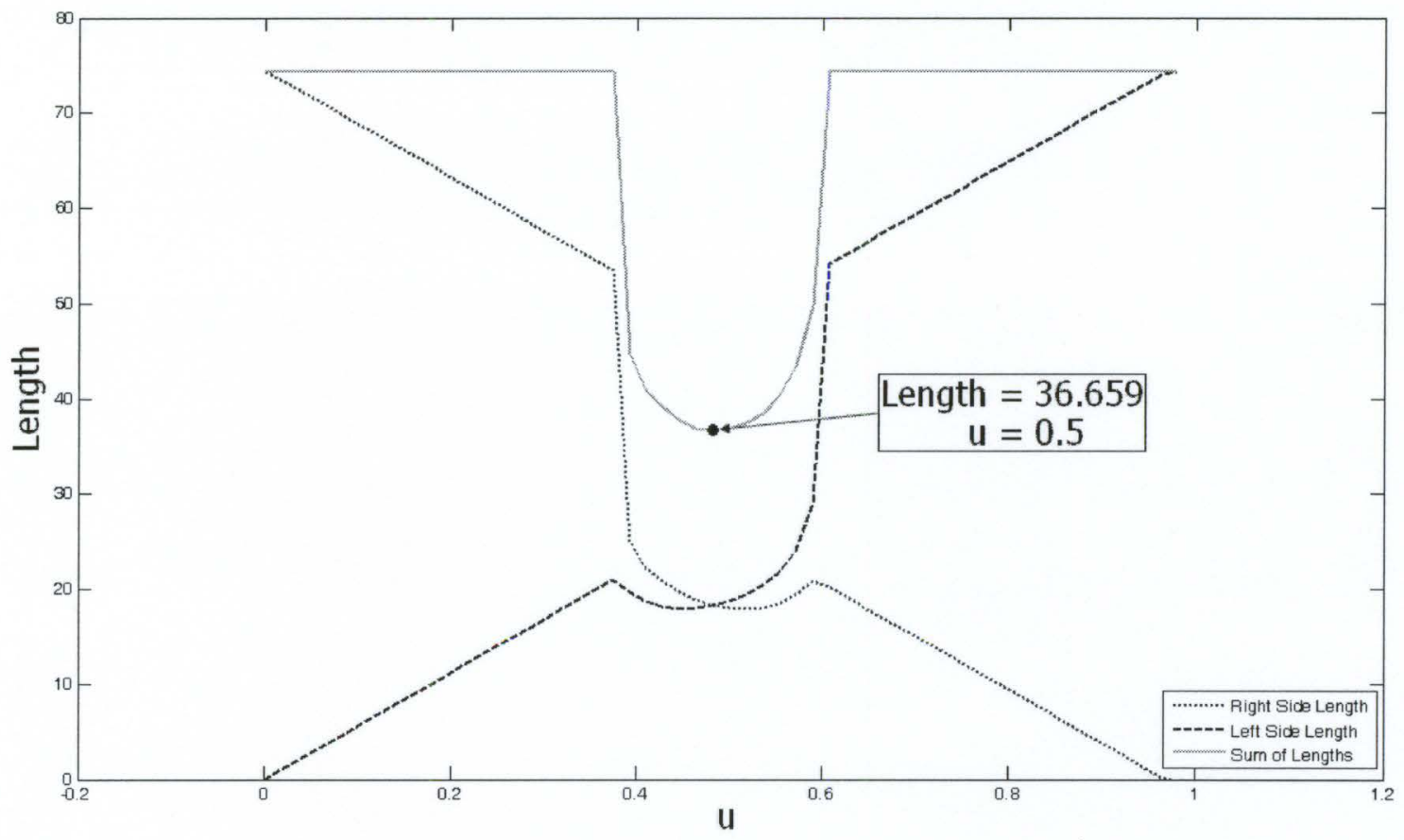

Figure 6.6: Star Shaped Cavity - Results of Tool Length Search 
According to the algorithm, the maximum centre line length is $36.6 \mathrm{~mm}$. The centre line of this tool, from which the length was calculated, is tangent at the centre of the convex curve $(\mathrm{u}=0.5)$. Fig. 6.7 shows the largest tool computed by the algorithm, which has a length of $46.6 \mathrm{~mm}$. Due to symmetry, this result occurs along all convex curves.

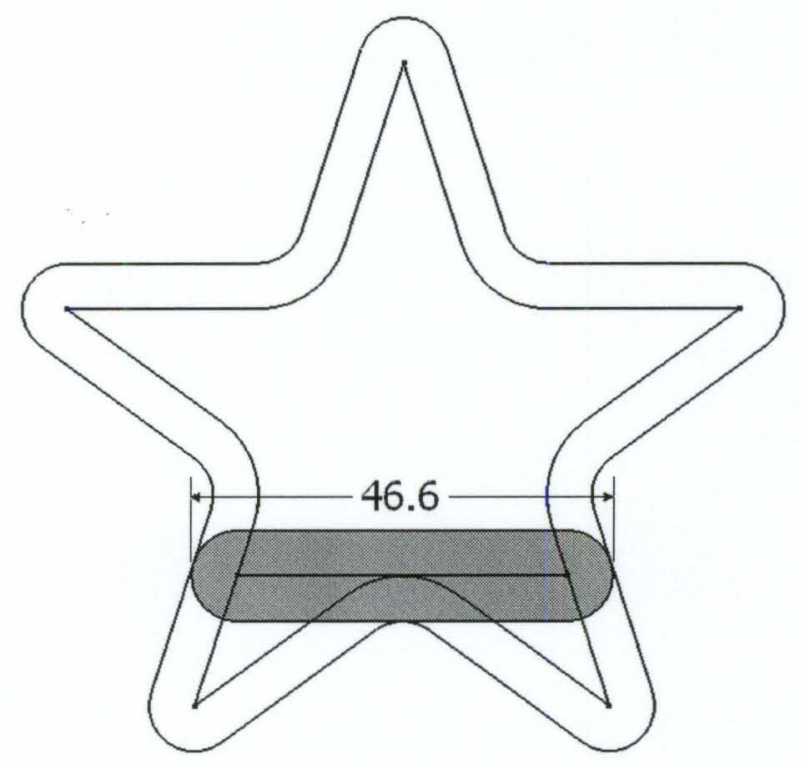

Figure 6.7: Star Shaped Cavity - Maximum Calculated Tool Size

Comparing the values obtained from the tests resulted in a maximum tool length of $46.6 \mathrm{~mm}$. Therefore, the tool used for rough ED milling of the star shaped cavity must have a width equal to $10 \mathrm{~mm}$ and a length less then $46.6 \mathrm{~mm}$. To demonstrate the flexibility of this approach, the chosen tool was smaller than maximum and was $10 \mathrm{~mm}$ in width and $25 \mathrm{~mm}$ in length. In comparison to an equivalent cylindrical tool of $10 \mathrm{~mm}$ diameter, this tool has approximately $200 \%$ more frontal area. It should be noted that the length of the tool was smaller than the maximum permissible length. A tool having the same width and the maximum permissible length results in a $450 \%$ increase in frontal area over the equivalent cylindrical tool. Fig. 6.8 shows the selected tool and an equivalent cylindrical tool. 

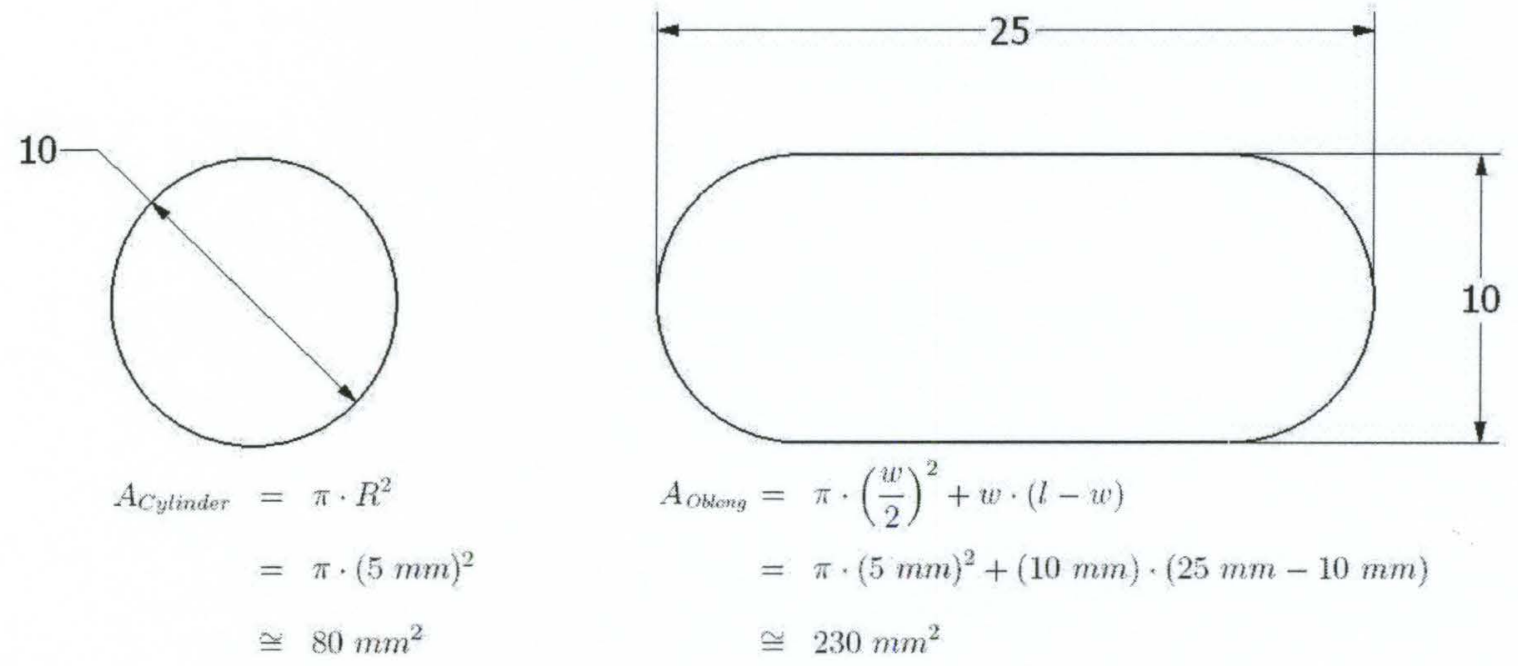

Figure 6.8: Star Shaped Cavity - Tool Size Comparison

In addition to increased power capacity, a larger frontal area reduces the number of passes required to completely machine a layer of the cavity. Applying the offset approach presented in Chapter 5 suggests that the selected tool can achieve this in one pass. Fig. 6.9 shows the closed loops contained within the profile after offsetting it by the tool length. The direction of offset for each curve is also indicated. None of the closed loops are composed entirely of curves having offset directions which point toward the interior of the loop. Therefore, only one pass per layer is required. An equivalent cylindrical tool requires at least three passes to fully machine a single layer.

To generate the tool path such that the tool sweeps the entire layer in one pass, the profile must first be offset by the tool width. The centre of the arc which composes the tools end is constrained to the offset curve and driven along it in the counter-clockwise direction. As the tool progresses, its orientation is adjusted between the critical points. The critical points are indicated in the tool path figures by dots on the offset curves. 
Master's Thesis - I. J. Mikesic McMaster - Mechanical Engineering

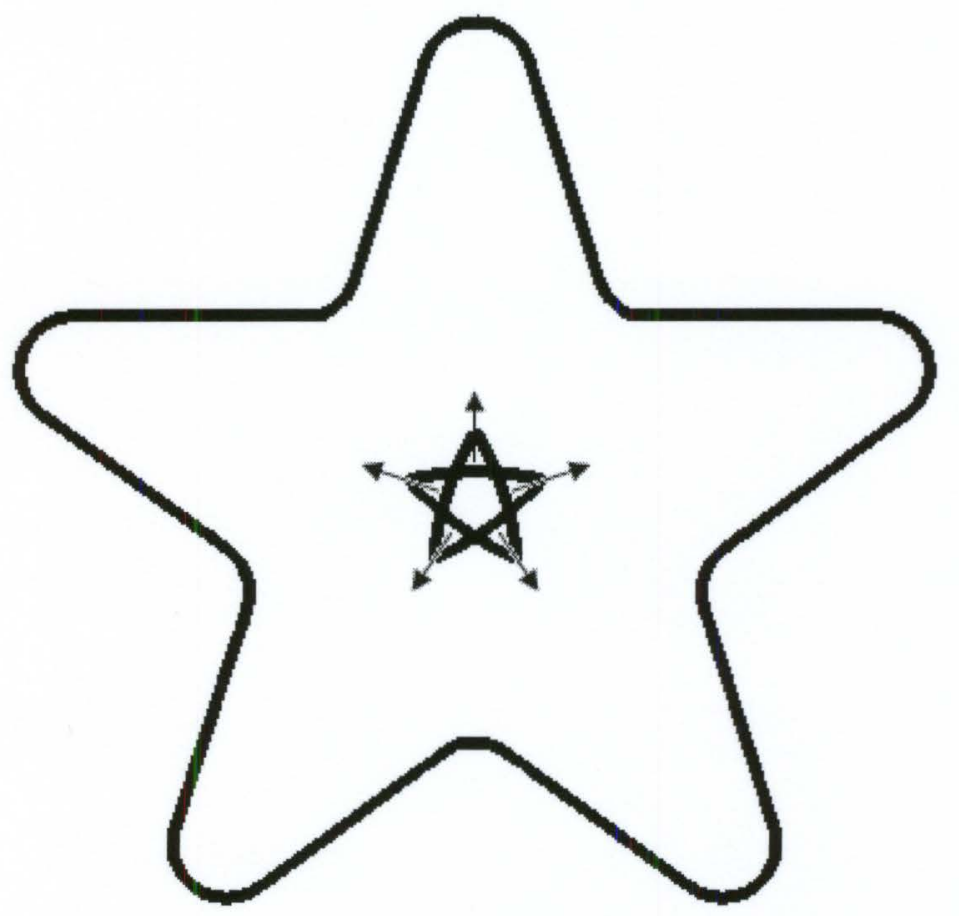

Figure 6.9: Star Shaped Cavity - Profile Offset by Tool Length

Fig. 6.10 and Fig. 6.11 show the tool path for two prongs of the star. A series of tool positions are presented showing the progression of the tool along each segment. Numbers indicate the start/end position of the tool for each tool path segment and arrows indicate the direction in which the tool is traveling. The offset profile, to which the tool tip is constrained, is also shown. Consecutive rotation of segments (a) - (d) by $72^{\circ}$ results in the complete tool path for a given layer. The tool path is executed in the opposite direction after each layer. A similar tool path, for the largest calculated tool, is shown in Figs. 6.12 and 6.13. 


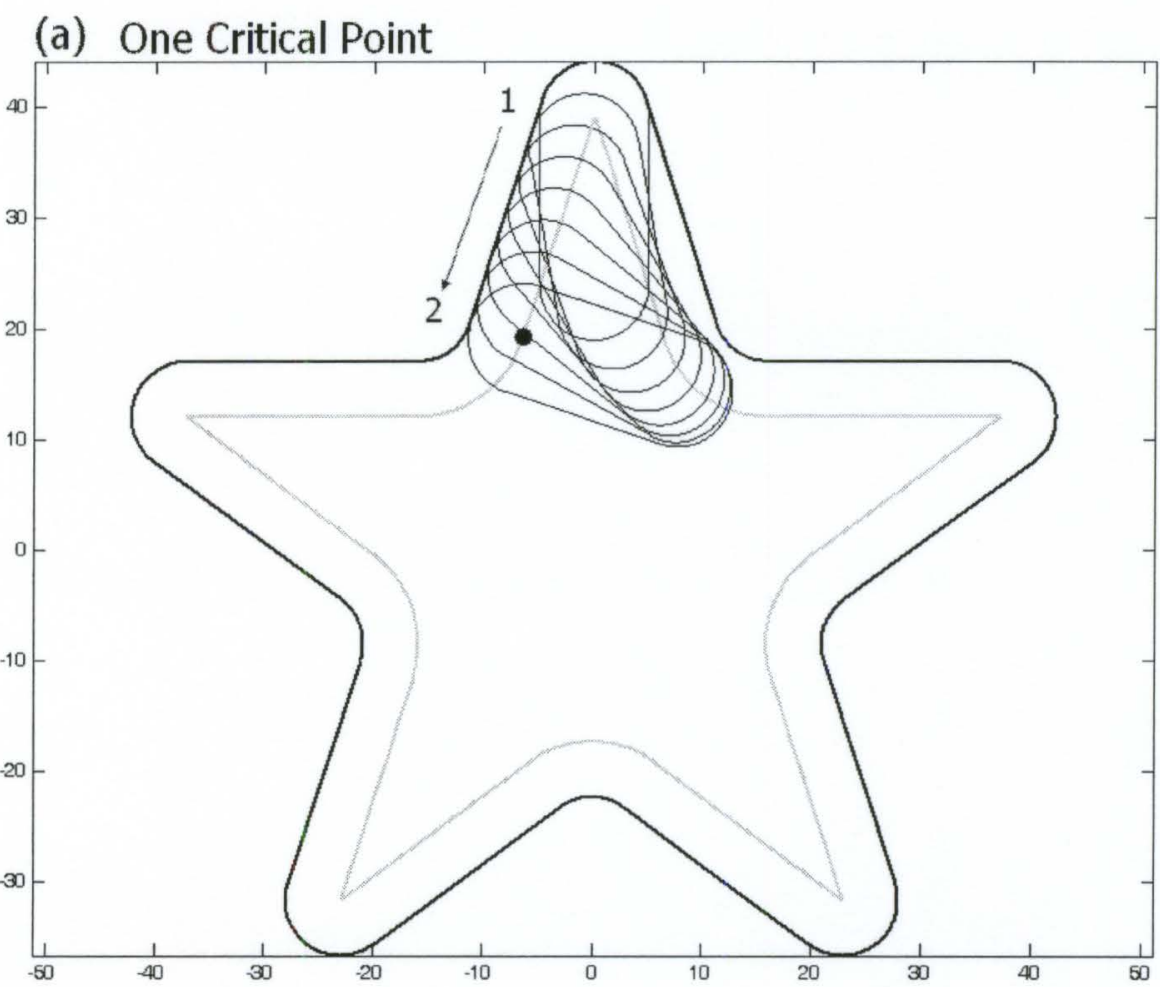

(b) No Critical Points

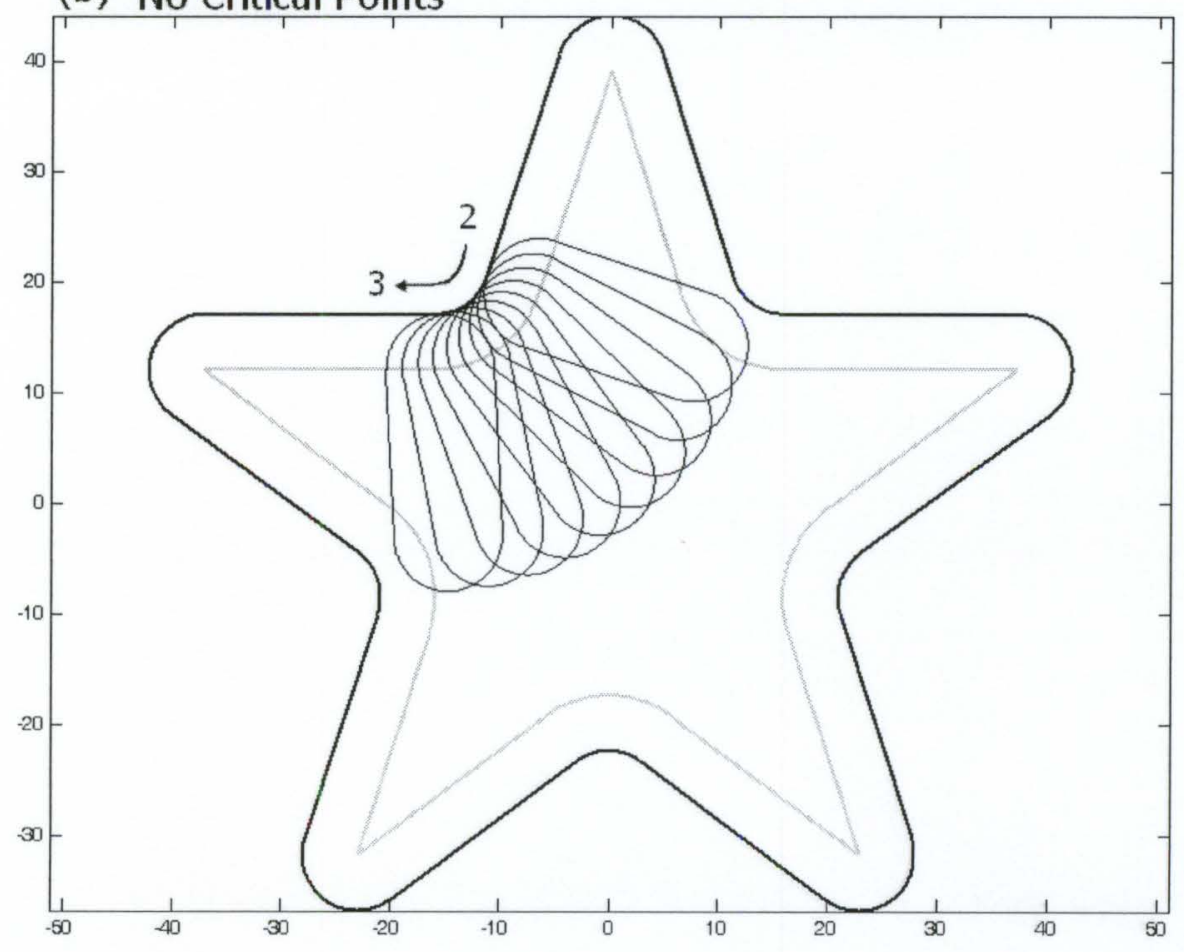

Figure 6.10: Star Shaped Cavity - Tool Path Segments A \& B 
(c) One Critical Point

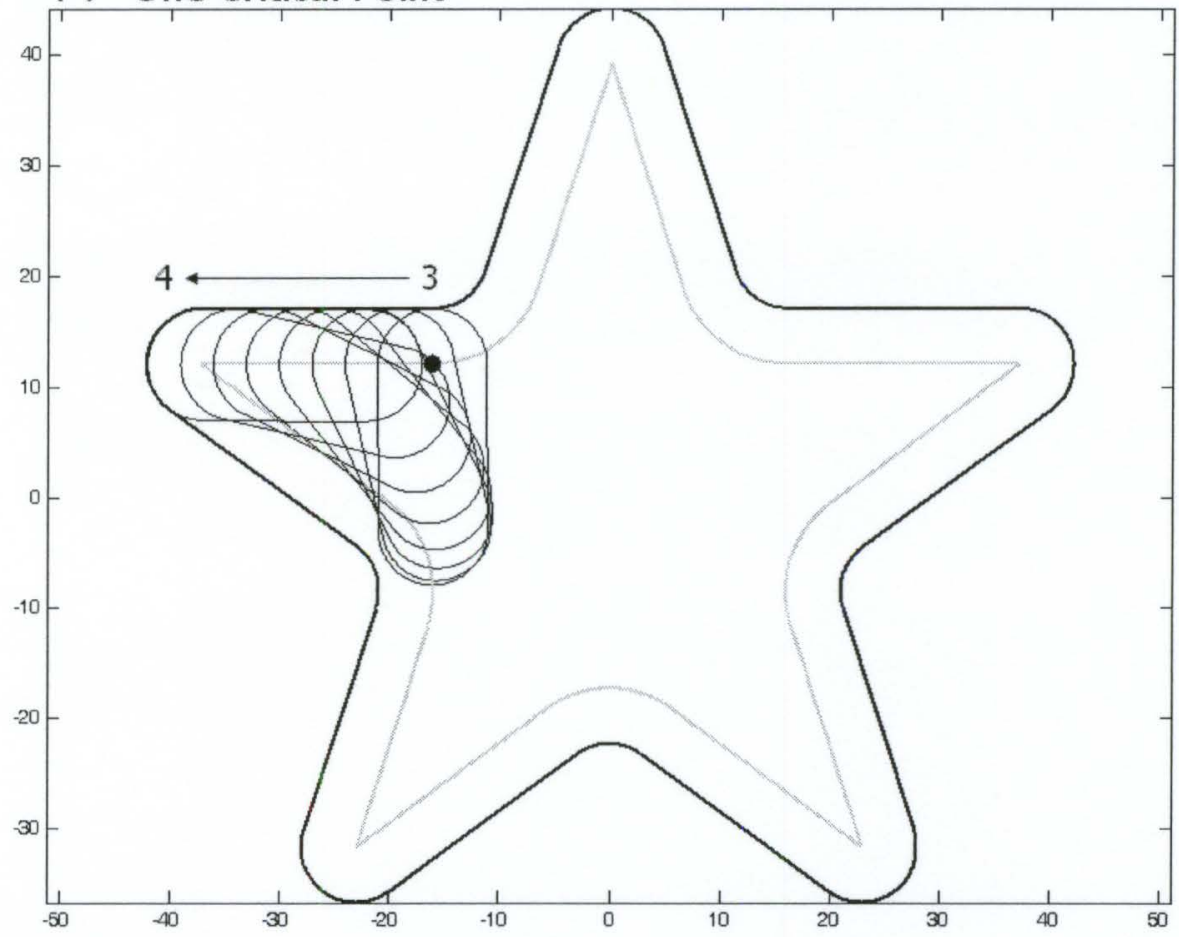

\section{(d) One Critical Point}

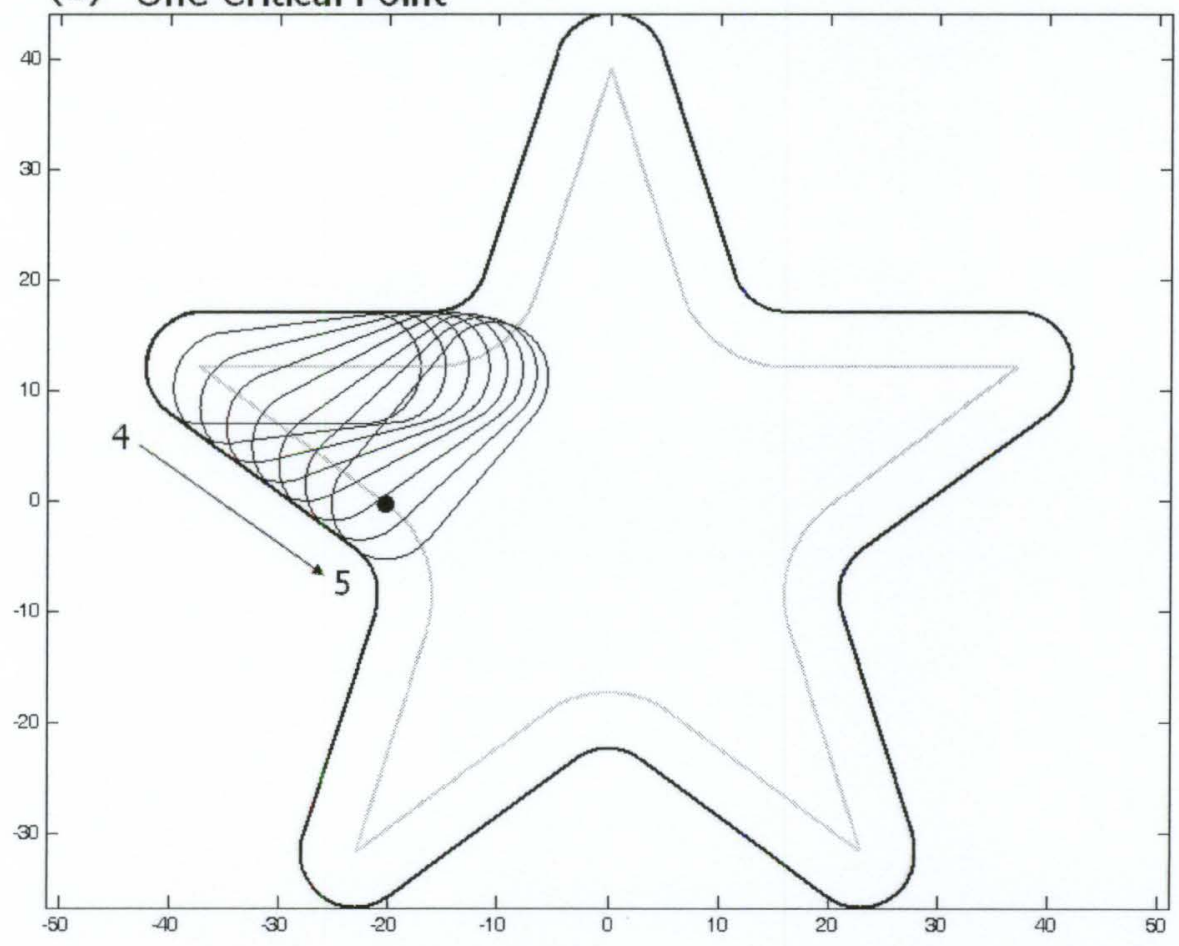

Figure 6.11: Star Shaped Cavity - Tool Path Segments C \& D 
(a) One Critical Point

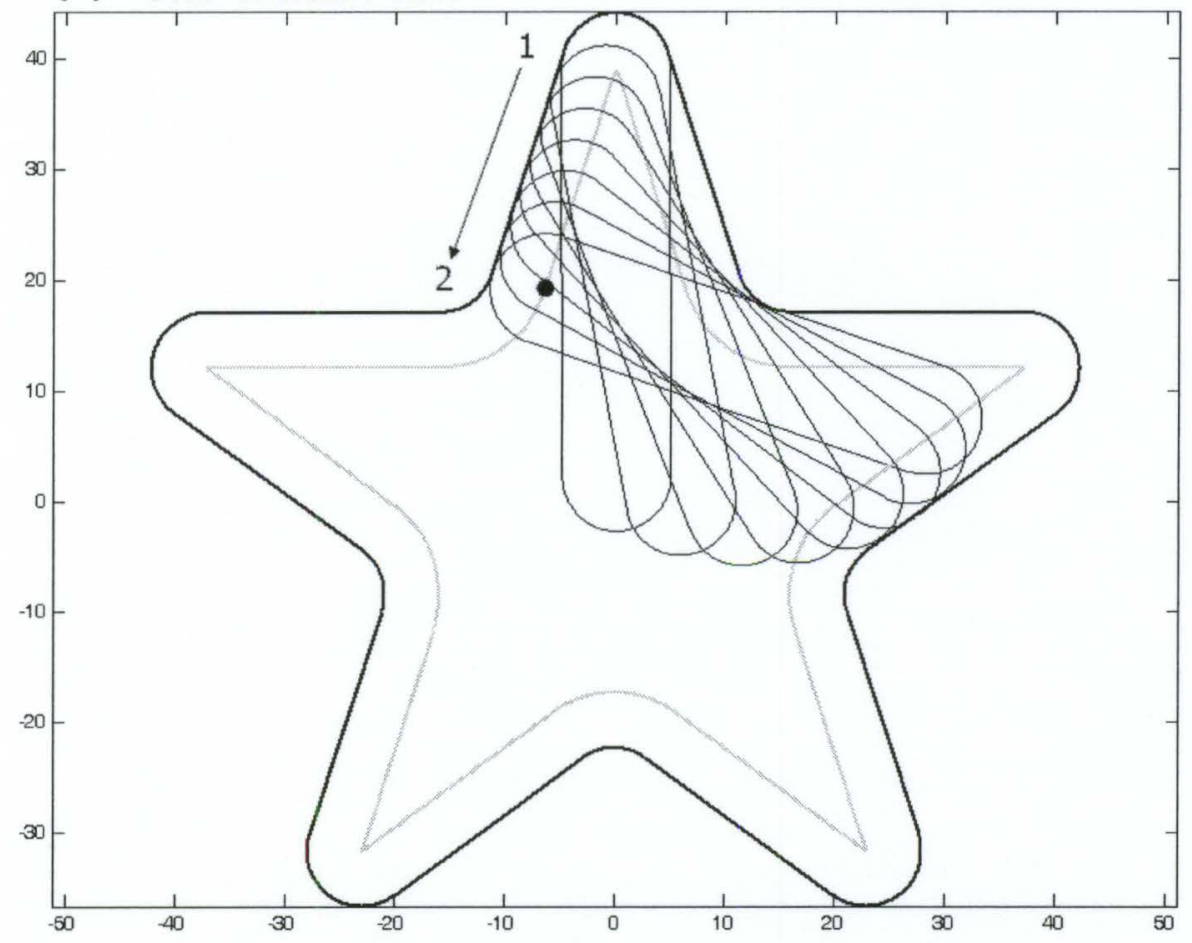

(b) One Critical Point

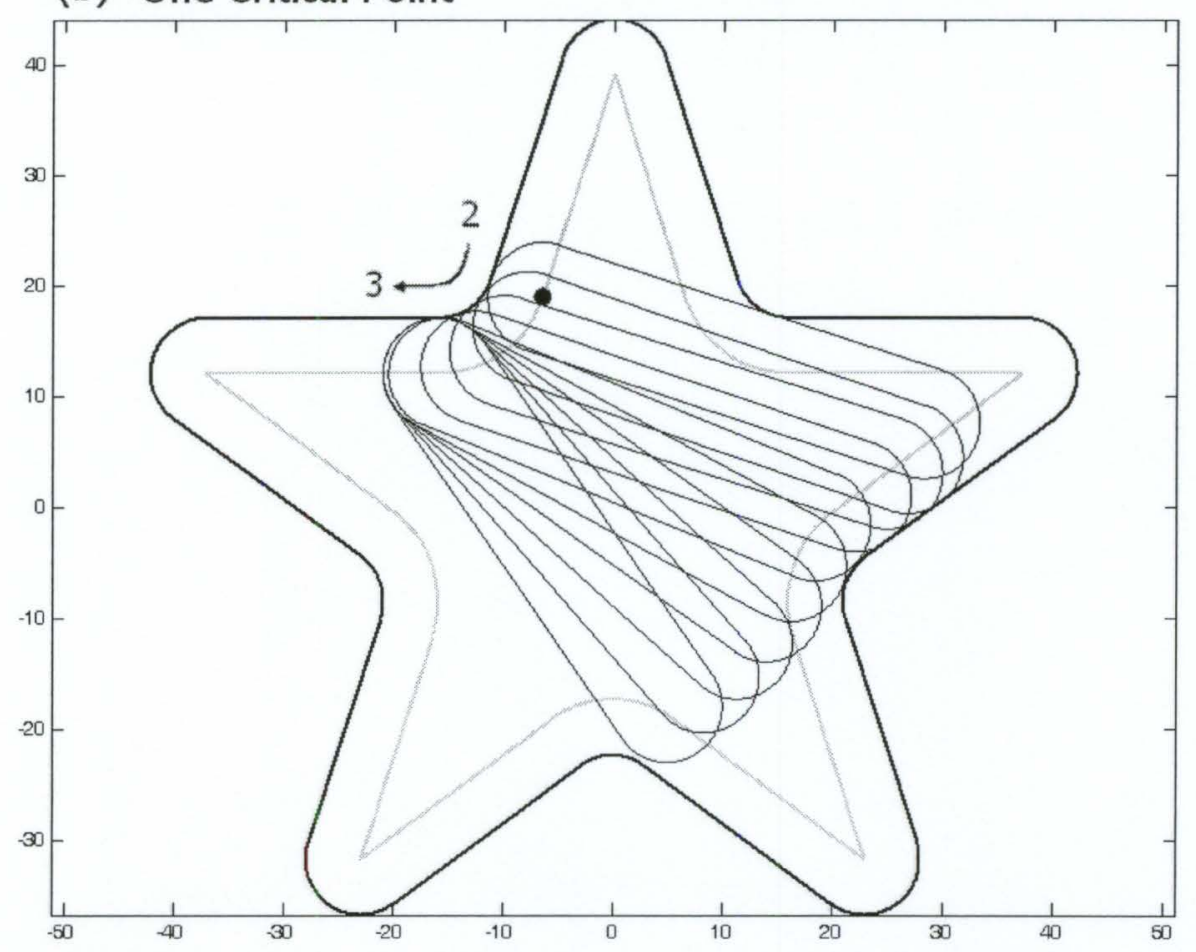

Figure 6.12: Star Shaped Cavity (Maximum Tool Size) - Tool Path Segments A \& B 


\section{(c) No Critical Points}

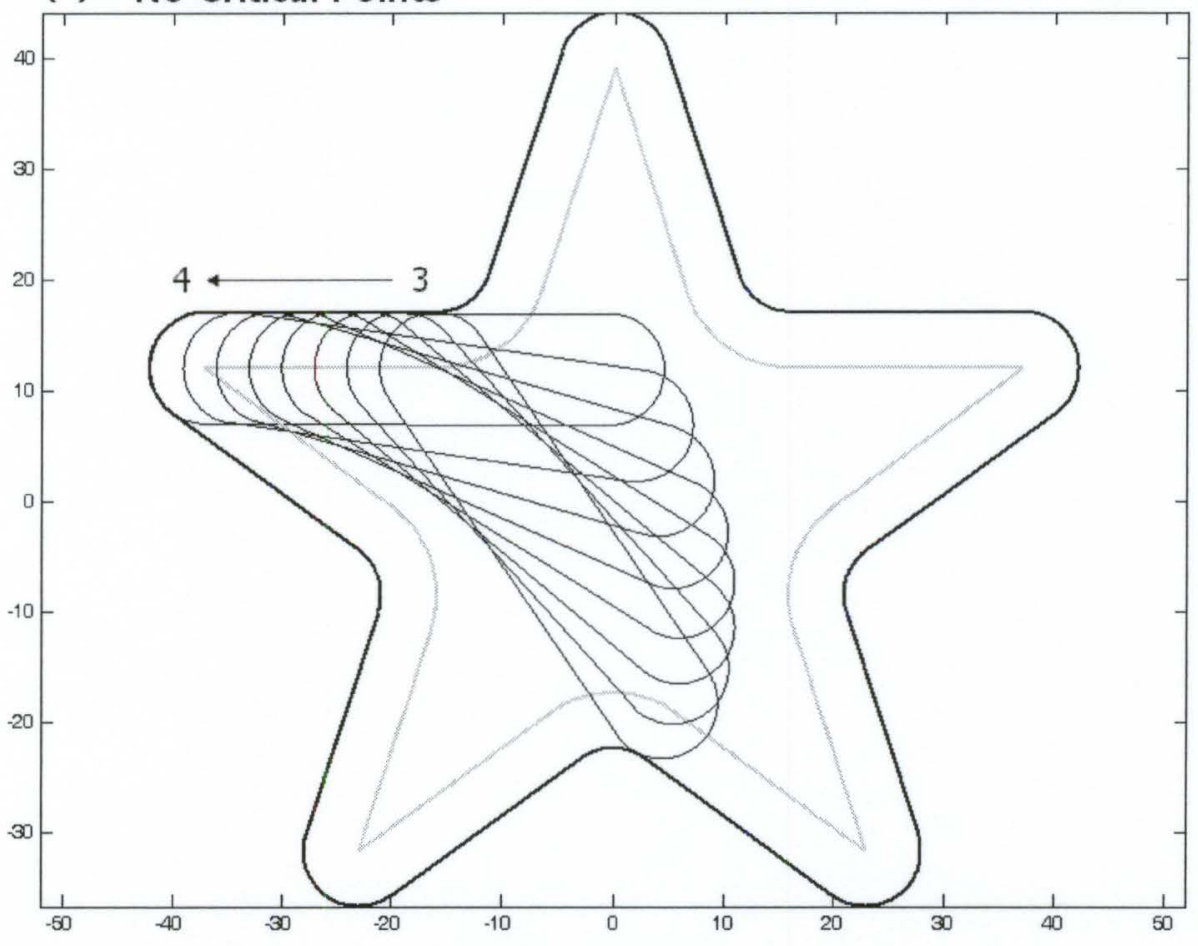

(d) One Critical Point

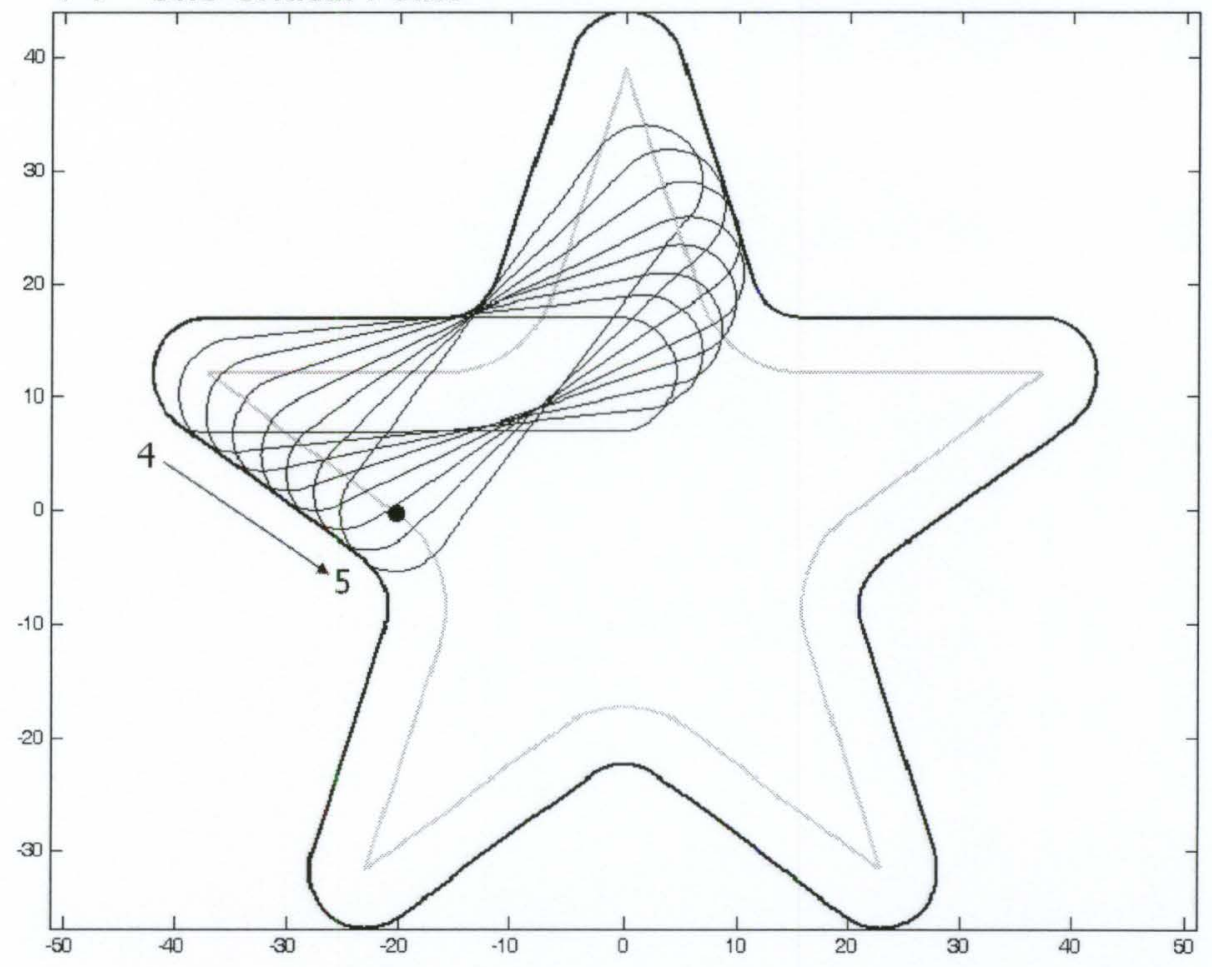

Figure 6.13: Star Shaped Cavity (Maximum Tool Size) - Tool Path Segments C \& D 
Machining of the star shaped cavity was performed on the AGIE IMPACT 2, a 4-axis CNC EDM machine tool. The tool used was made of copper and manufactured on a 3-axis CNC milling machine. Fig. 6.14 shows the tool. The front face of the tool was trued on a milling machine using the System $3 \mathrm{R}$ table chuck to hold it. This ensured that the front face of the tool was sufficiently flush with the workpiece.

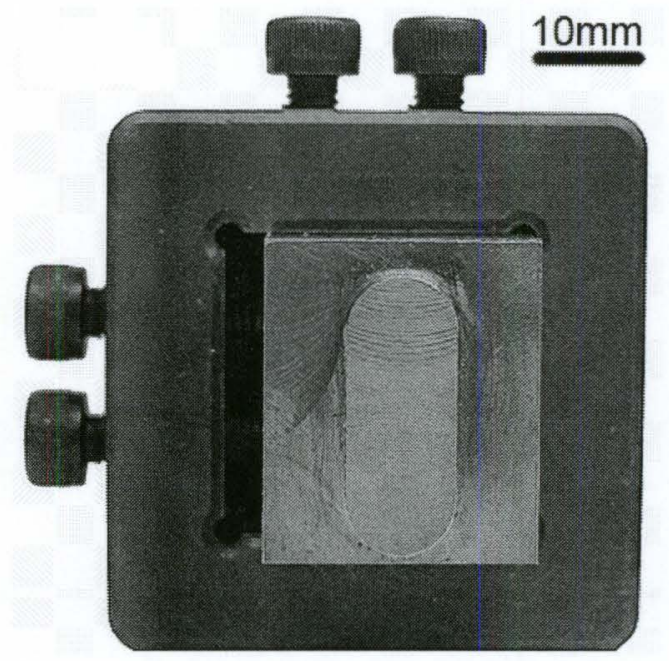

Figure 6.14: Tool used in ED Milling of the Star Shaped Cavity

Pulse parameters used during machining are shown in Table 6.2. The machining depth was $1 \mathrm{~mm}$ and the total machining time was approximately two hours. Flushing was induced by the motion of the tool with respect to the workpiece. Further flushing was provided by external jets. Fig. 6.15 shows the setup of the flushing jets. They were positioned perpendicular to each other on the workpiece surface.

\begin{tabular}{cc} 
Parameter & Value \\
\hline \hline Current $(\mathrm{A})$ & 21 \\
Voltage $(\mathrm{V})$ & 120 \\
Pulse On-Time $(\mu \mathrm{s})$ & 49 \\
Pulse Off-Time $(\mu \mathrm{s})$ & 49 \\
Polarity & +
\end{tabular}

Table 6.2: Star Shaped Cavity - Pulse Parameters 


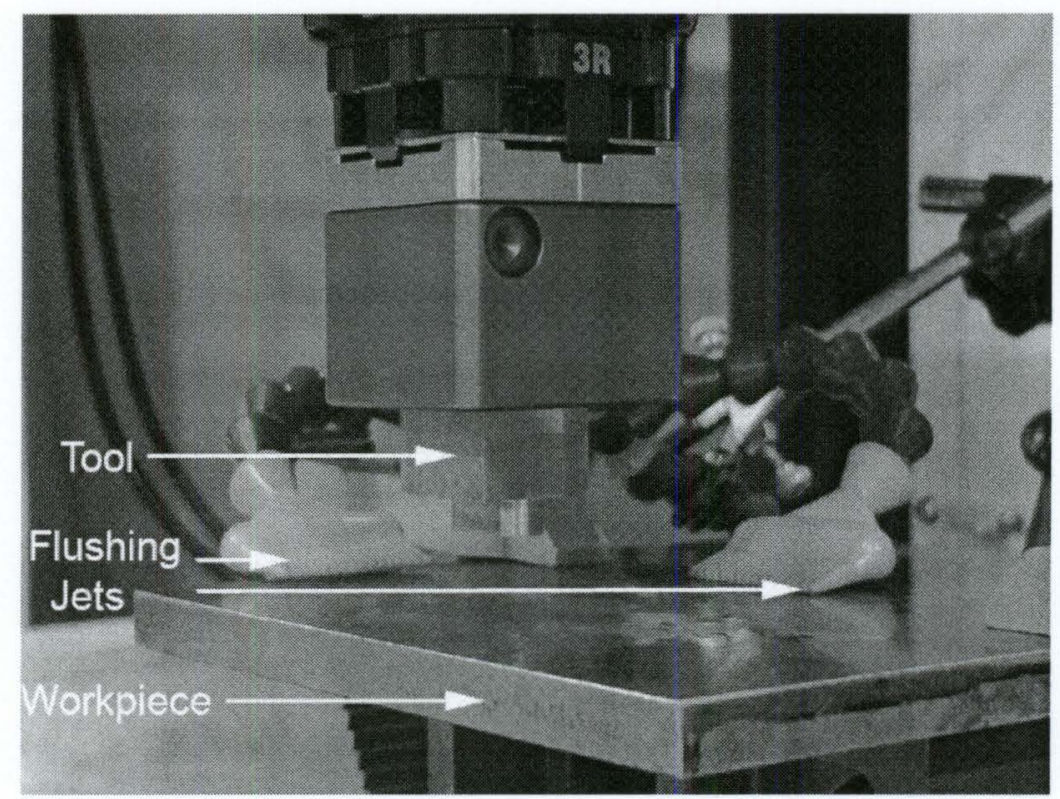

Figure 6.15: Experimental Setup

Fig. 6.16 shows the machined cavity. Due to tool wear and an overlapping tool path, the bottom surface of the cavity was not flat. However, this deviation would be corrected in semi-finishing and finishing operations.

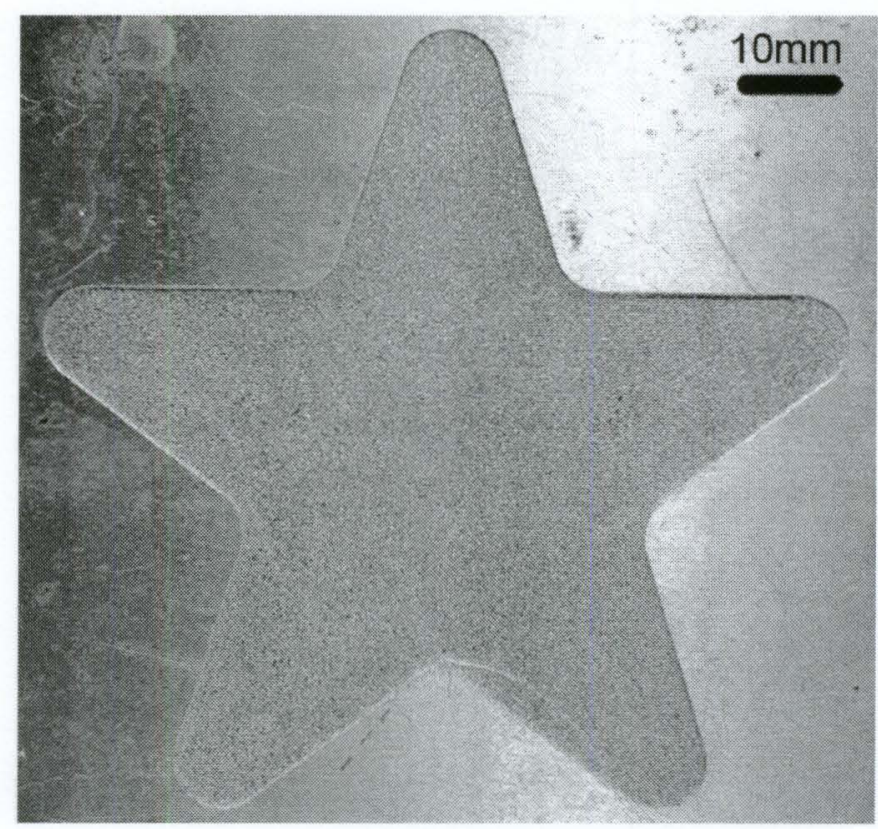

Figure 6.16: Star Shaped Cavity Machined by ED Milling with Tool Type 1 
Fig. 6.16 illustrates the success of this method in the machining of a star shaped cavity. Some of the benefits realized in this example were increased frontal area of the tool and the complete machining of a single layer in one pass. Furthermore, the flexibility of the method was demonstrated by using a tool of less than optimal length.

\subsection{Example 2 - Gear Shaped Cavity}

A gear shaped cavity is presented in this section to demonstrate the algorithms in this work as they apply to a cavity profile of type 3 . The dimensioned cavity profile is in Fig. 6.17. Dimensions of length are expressed in $\mathrm{mm}$ and angular dimensions are in degrees. Due to symmetry, only one tooth of the gear is dimensioned.

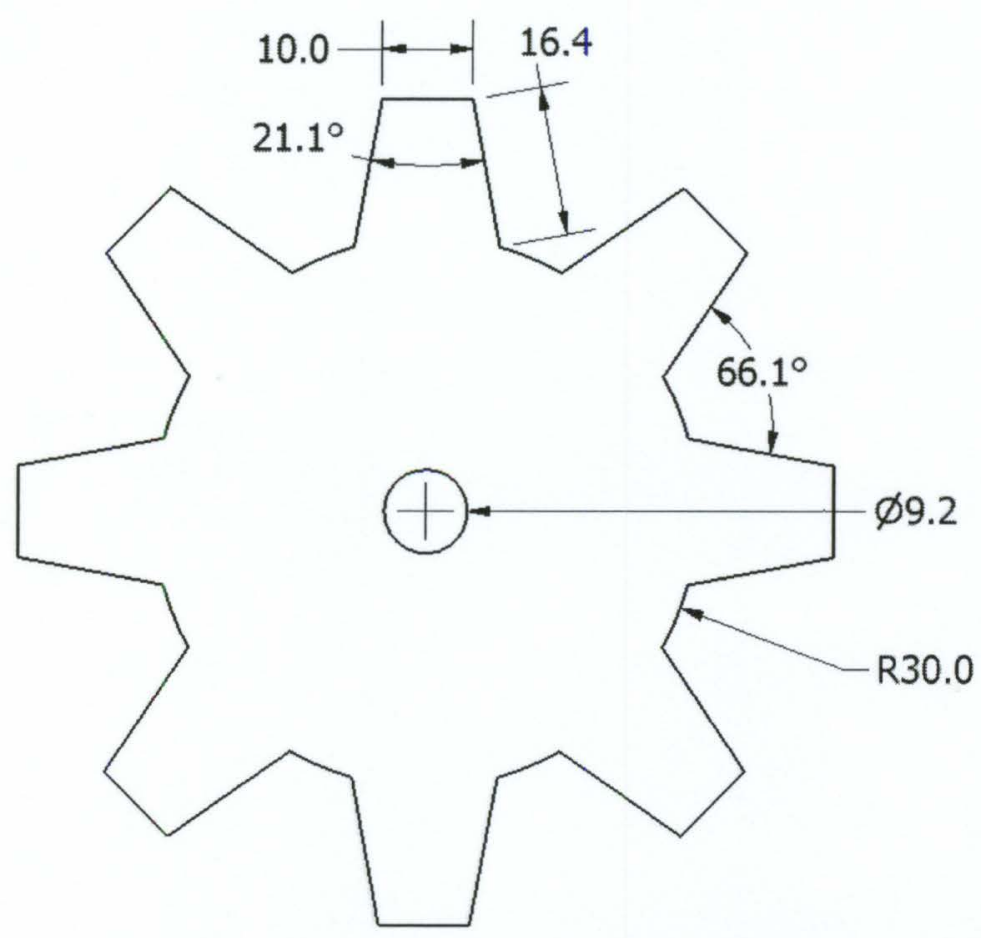

Figure 6.17: Gear Shaped Cavity (Dimensions in $\mathrm{mm}$ ) 
The algorithm for tool selection confirms that the profile is of type 3. Fig. 6.18 shows the curve composition of the cavity profiles. Curves are numbered in a counterclockwise fashion and their endpoints are indicated by dots. Tangent continuity is expressed by the use of symbol. Results of testing for tangent continuity between curves are shown in Table 6.3. Concave curve pairs are highlighted. It is to be noted that the circular arcs composing the root diameter of the gear are also concave.

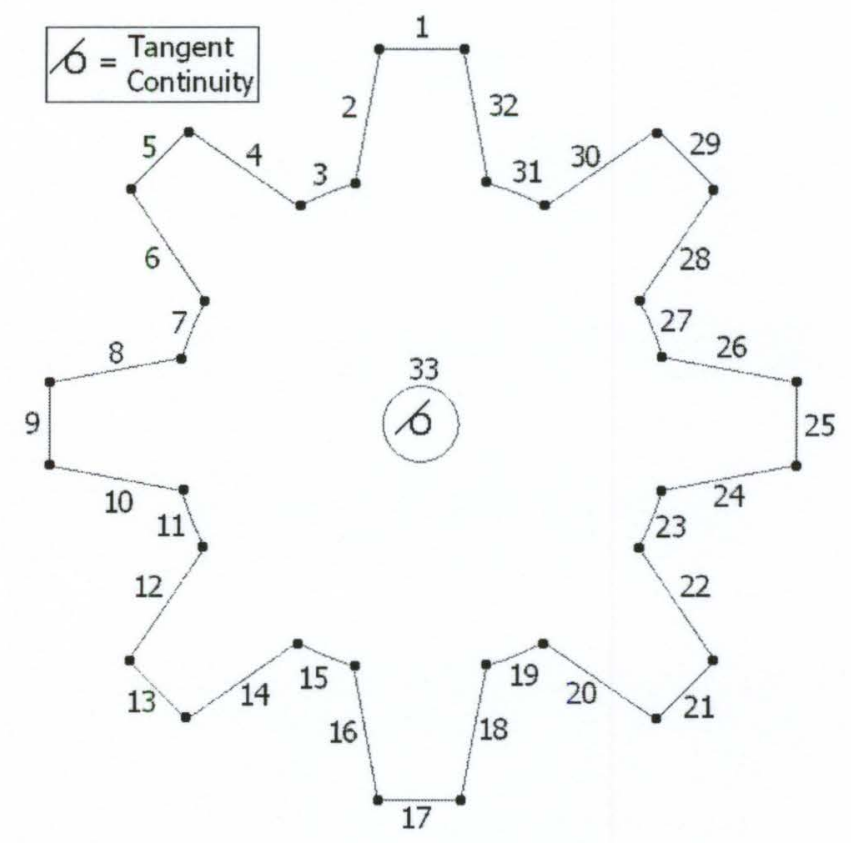

Figure 6.18: Curves Composing the Profile of the Gear Shaped Cavity

\begin{tabular}{cc|cc|cc} 
Curve Pair & Tangent Continuous & Curve Pair & Tangent Continuous & Curve Pair & Tangent Continuous \\
\hline \hline $1-2$ & No & $12-13$ & No & $23-24$ & No \\
$2-3$ & No & $13-14$ & No & $24-25$ & No \\
$3-4$ & No & $14-15$ & No & $25-26$ & No \\
$4-5$ & No & $15-16$ & No & $26-27$ & No \\
$5-6$ & No & $16-17$ & No & $27-28$ & No \\
$6-7$ & No & $17-18$ & No & $28-29$ & No \\
$7-8$ & No & $18-19$ & No & $29-30$ & No \\
$8-9$ & No & $19-20$ & No & $30-31$ & No \\
$9-10$ & No & $20-21$ & No & $31-32$ & No \\
$10-11$ & No & $21-22$ & No & $32-1$ & No \\
$11-12$ & No & $22-23$ & No & 33 & Yes
\end{tabular}

Table 6.3: Gear Shaped Cavity - Tangent Continuity Search Results 
No pairs of curves are tangent continuous in this profile confirming that the profile is of type 3 . Therefore, the algorithm suggests the use of tool type 3 . However, to demonstrate the flexibility of the tool set, a type 2 tool was selected. In this case, the tool results in a larger frontal area.

To determine the width of the tool, the MMAT was computed. The minimum value of the radius function was obtained as a possible candidate for defining the tool width. Since the cavity profile is of type 3 , the feature of minimum length was also determined as a candidate for defining tool width. This feature occurs at the edge of each gear tooth. It should be noted that the tapers at each tooth contain no lines which required removal from the search. Fig. 6.19 shows the MMA, the circle of minimum radius (shaded) and the feature of minimum length (dashed).

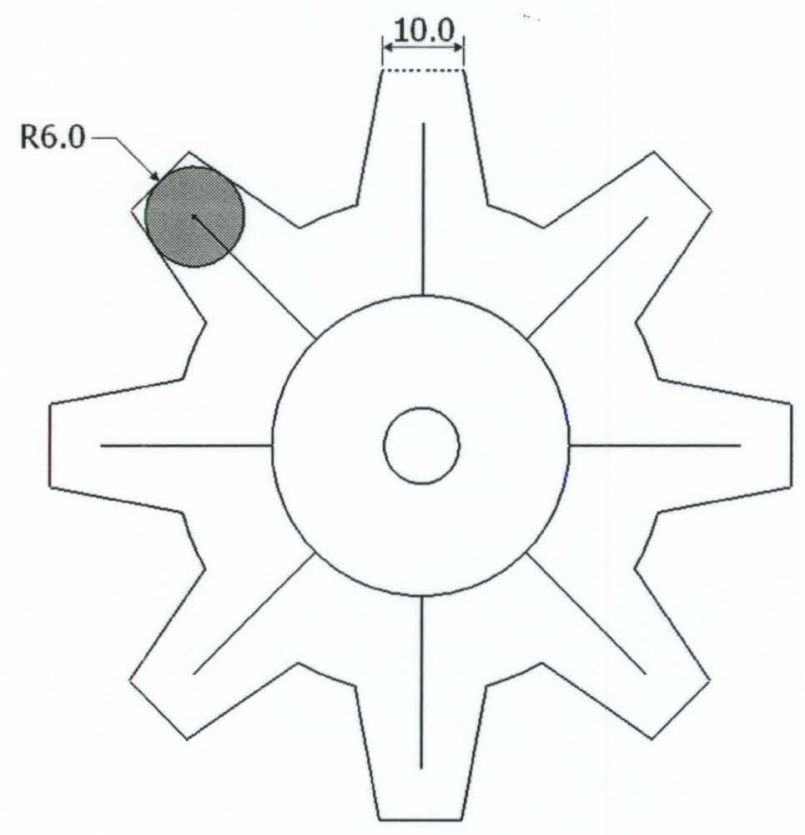

Figure 6.19: Gear Shaped Cavity - Features of Minimum Length and Radius

The feature of minimum length defined the maximum permissible tool width for this cavity. Therefore, a tool width less than or equal to $10 \mathrm{~mm}$ was required. The selected tool width was the maximum, $10 \mathrm{~mm}$. 
The MIC of the cavity profile was determined as a possible solution for the maximum permissible tool length. Searching the radius function for its maximum produced the MIC shown in Fig. 6.20. Applying compensation to the length, such that a $10 \mathrm{~mm}$ wide tool fits within the maximum inscribed circle, resulted in a tool length of $25 \mathrm{~mm}$. The cavity profile contains no lines which are longer than the diameter of the MIC. Therefore, the lines contained in the profile offset do not define the tool length and need not be searched.

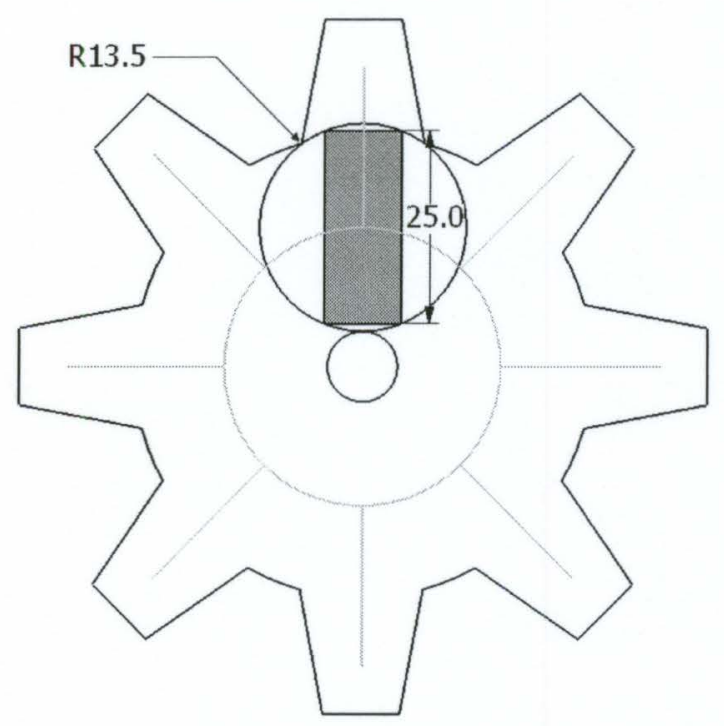

Figure 6.20: Gear Shaped Cavity - MIC and Corresponding Tool Length

Since the island in this cavity is convex, it was searched for tool length. Due to the symmetry of the cavity profile and the island, the results for one quarter of the island are presented. The region for which the results are presented and the corresponding maximum tool length are shown in Fig. 6.21.

Results for the centre line lengths along the entire region indicated in Fig. 6.21 are presented in Fig. 6.22. The minimum centre line length was $46.2 \mathrm{~mm}$ corresponding to a tool length of $55.30 \mathrm{~mm}$. In Fig. 6.22, the minimum values are indicated by dots. The last occurrence of the minimum centre line length is labeled. 


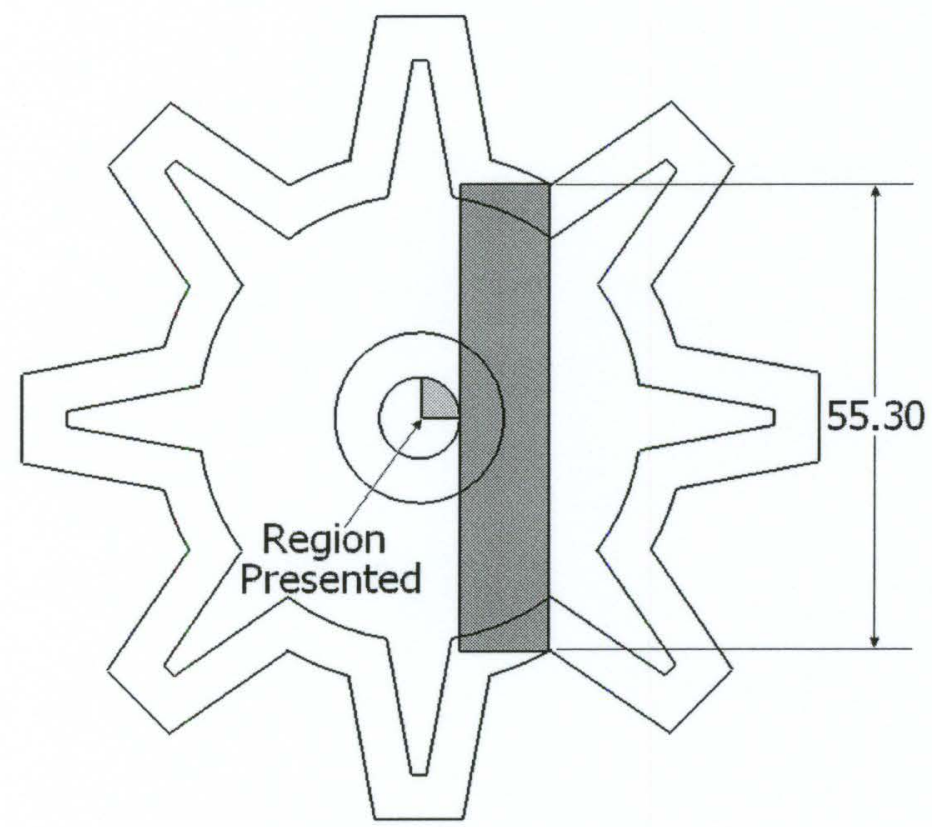

Figure 6.21: Gear Shaped Cavity - Convex Region and Resulting Tool Length

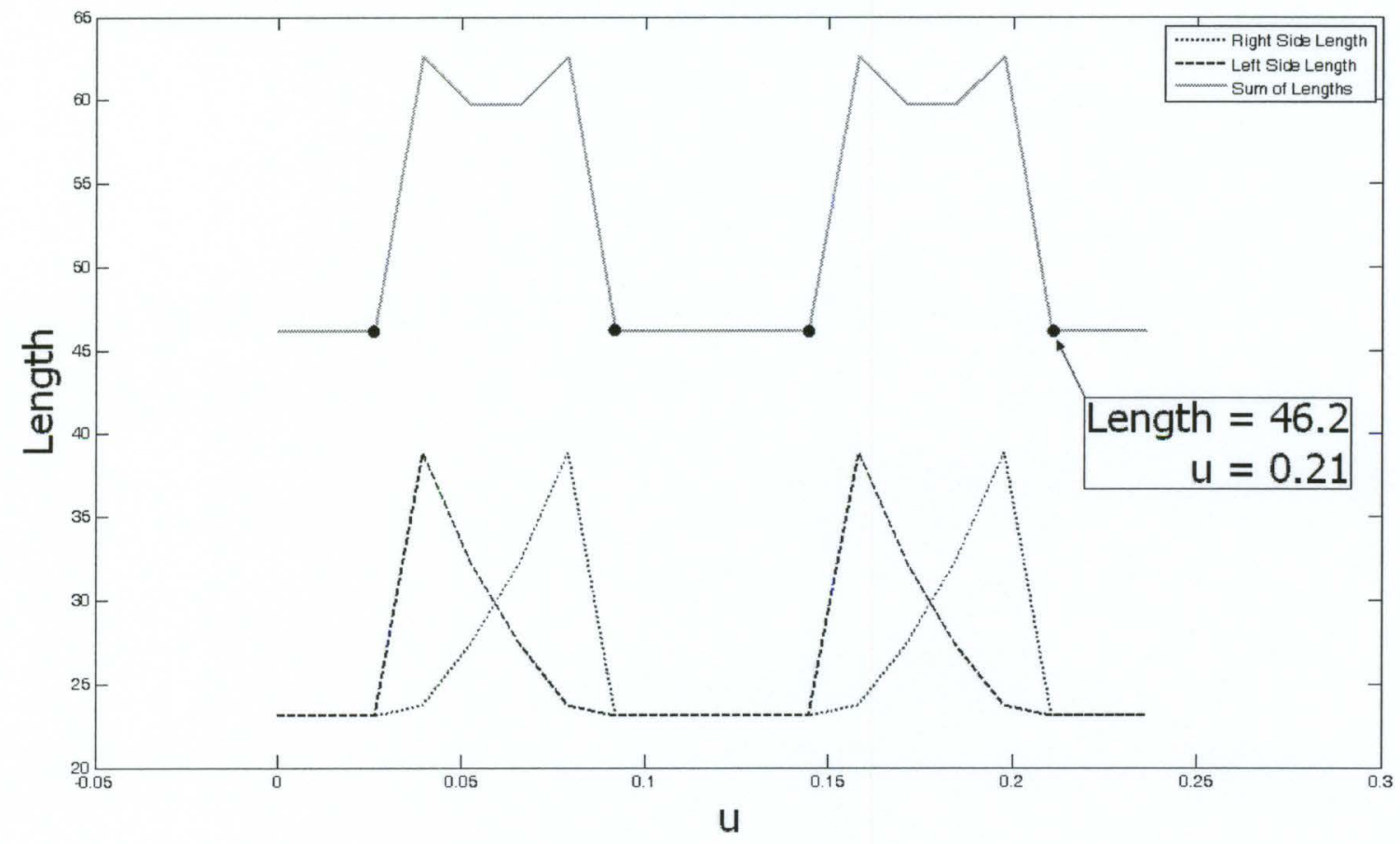

Figure 6.22: Gear Shaped Cavity - Tool Length Results for Convex Island 
Selecting the minimum length based on the values returned provided a tool length of $25 \mathrm{~mm}$. Therefore, the maximum tool size allowable for machining of the gear shaped cavity was $10 \mathrm{~mm}$ in width and $25 \mathrm{~mm}$ in length. A tool with length less than or equal to $25 \mathrm{~mm}$ may be used for the machining of this cavity. The tool length chosen was $25 \mathrm{~mm}$ and thus, the tool size was maximized.

Comparison of the selected tool with the equivalent square tool $(10 \mathrm{~mm} \times 10 \mathrm{~mm})$ indicates a $150 \%$ increase in area. The comparison in size between the tools and their respective areas are shown in Fig. 6.23.
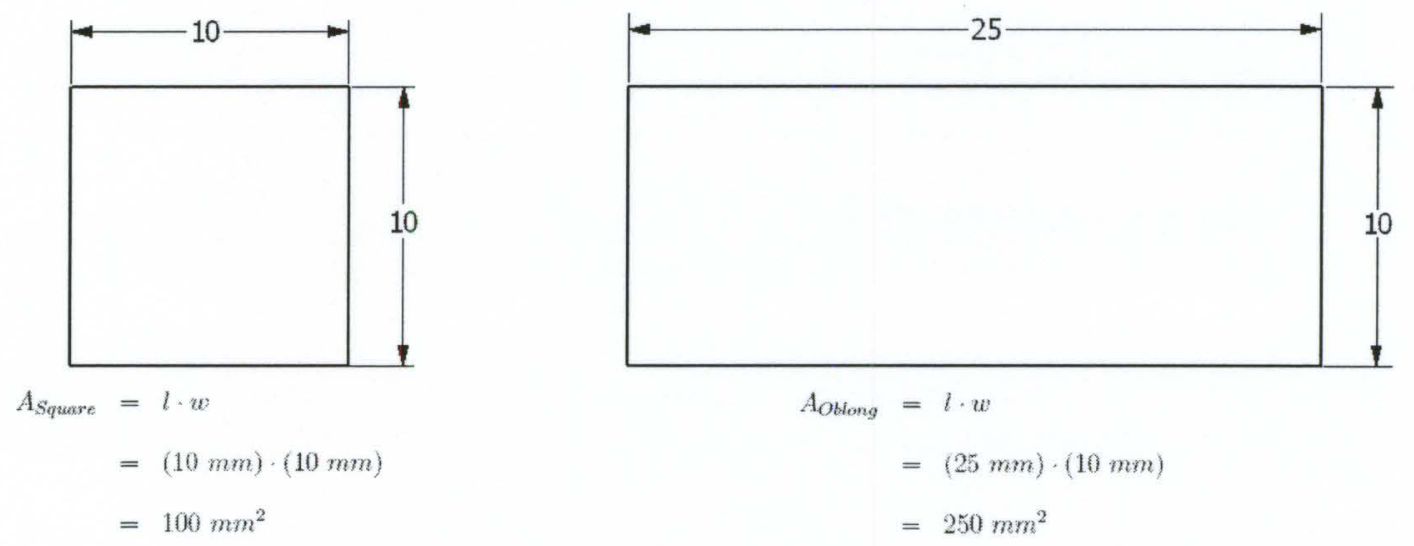

Figure 6.23: Gear Shaped Cavity - Tool Size Comparison

When the cavity profile was offset by the tool length, none of the closed loops formed were entirely contained with in the profile boundary. This confirmed that the selected tool could machine each layer of the cavity could in one pass.

To completely machine each layer in one pass, the tool was driven along the profile keeping at least one point on its tracing edge constrained to the profile at all times. In between endpoint, the tool orientation was adjusted so the tools sweeping edge and trailing point did not intersect the cavity profile. It should be noted that this profile contained no critical points. Fig. 6.24 and Fig. 6.25 show the tool path for two teeth of the gear. 
(a)

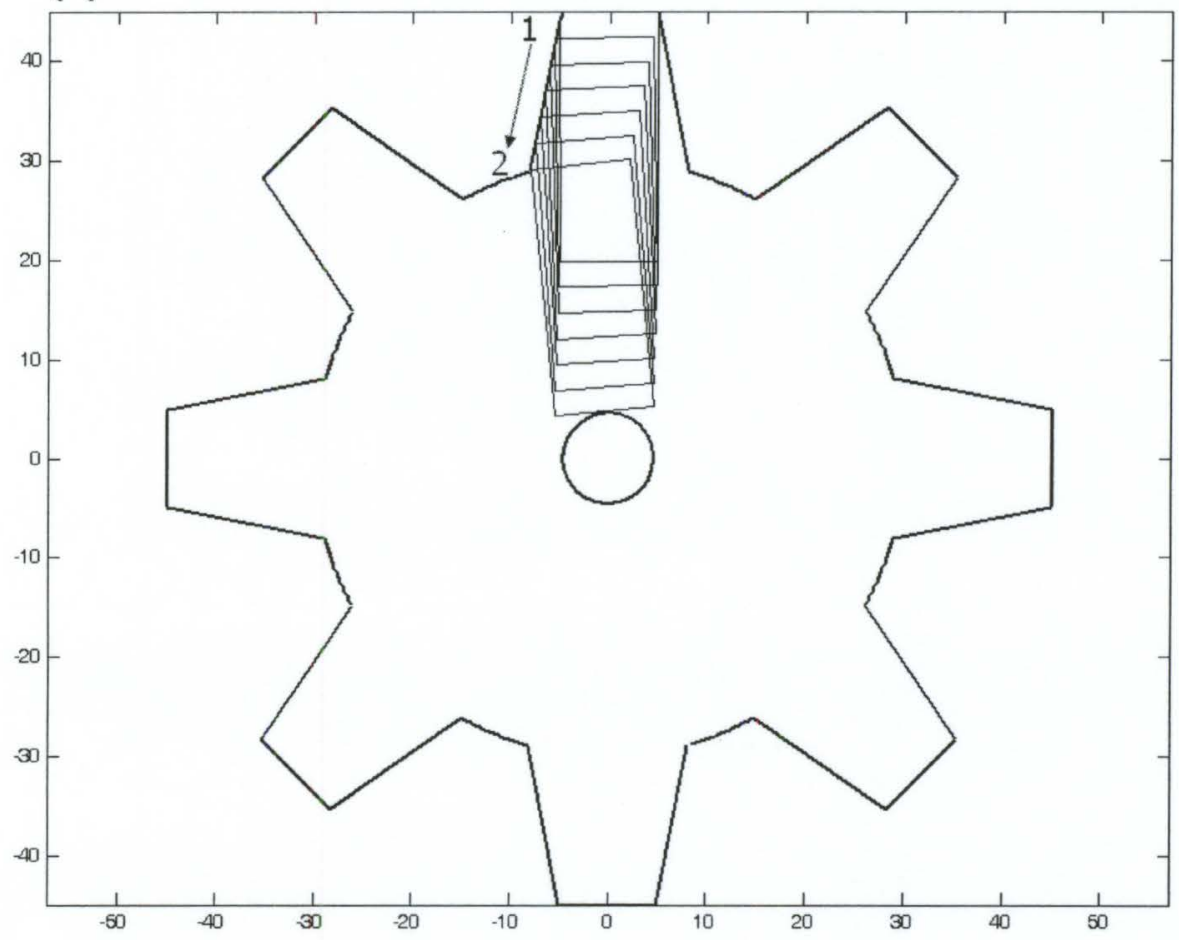

(b)

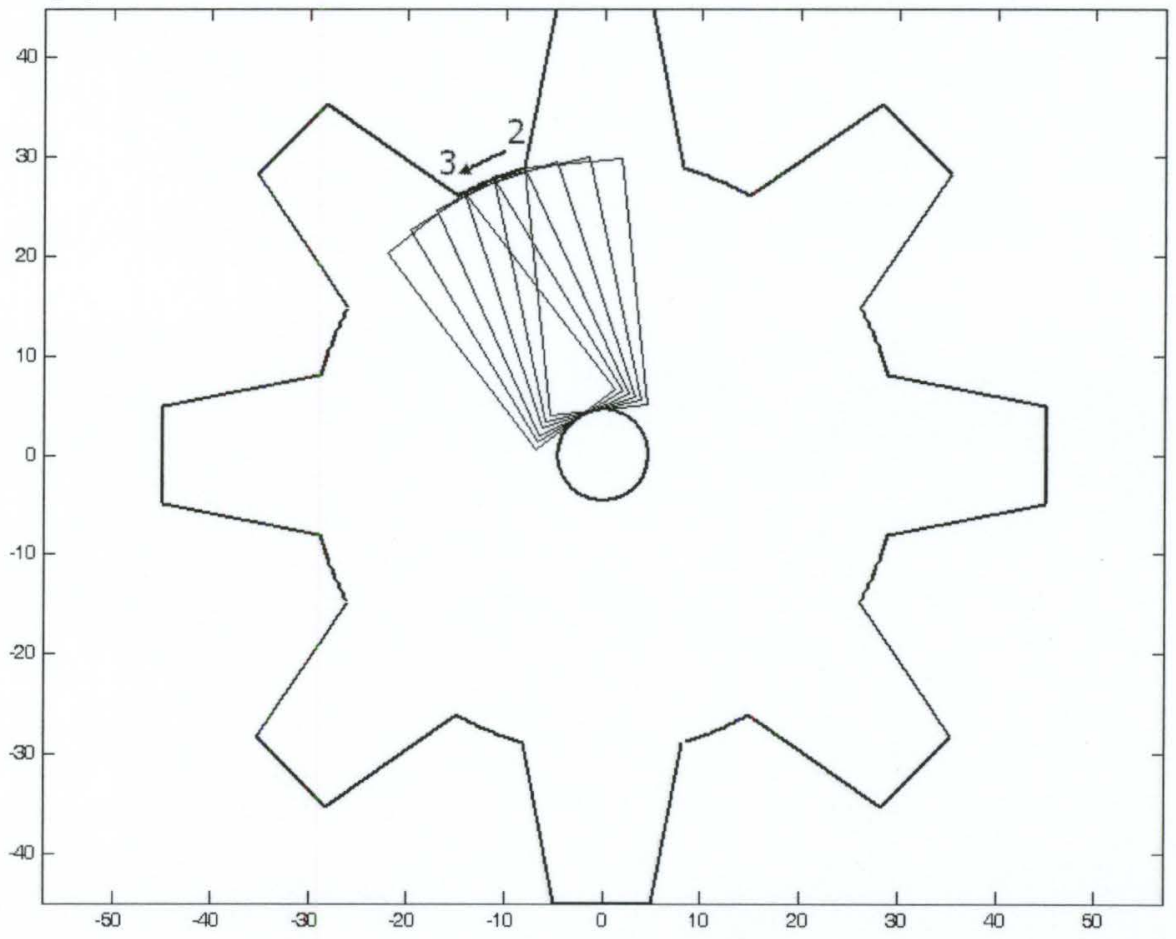

Figure 6.24: Gear Shaped Cavity - Tool Path Segments C \& D 
(c)

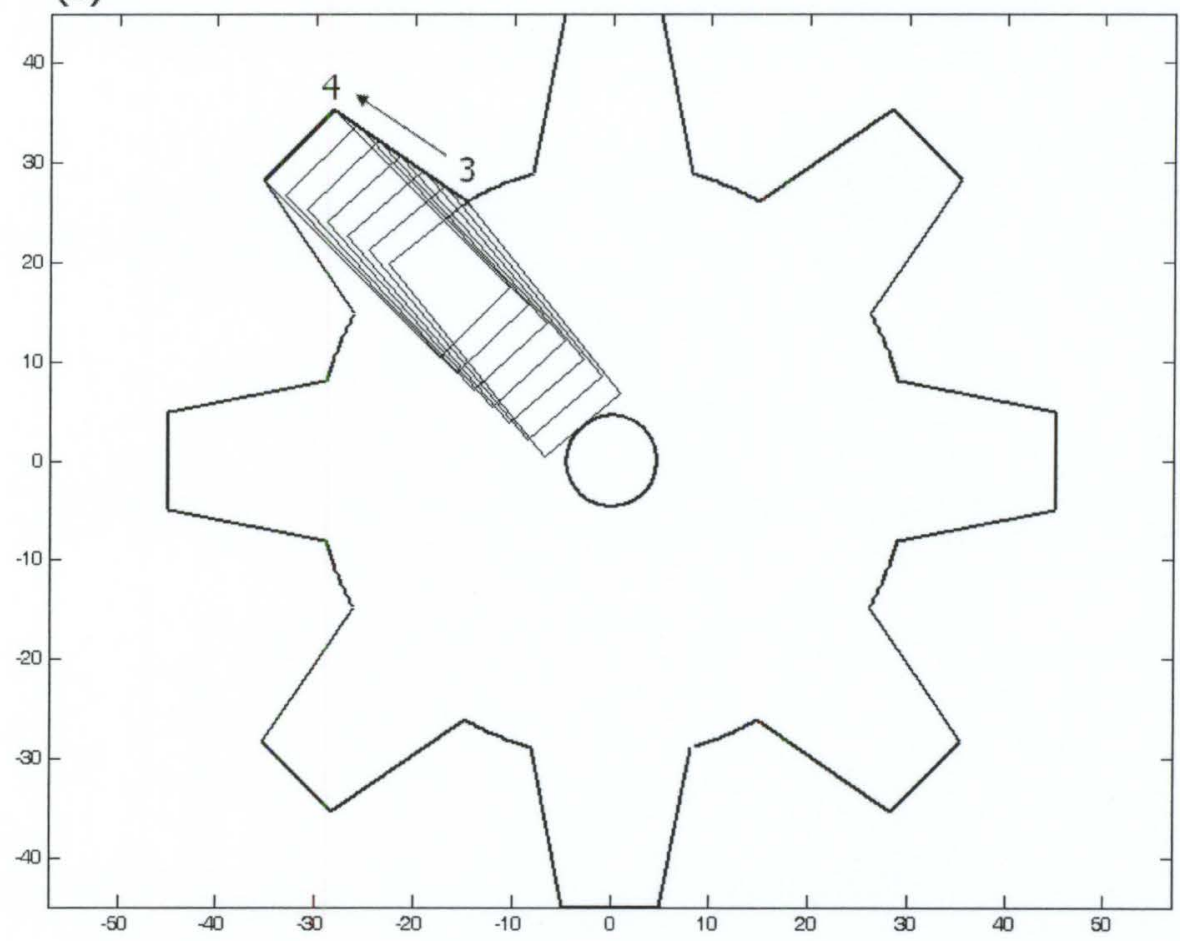

(d)

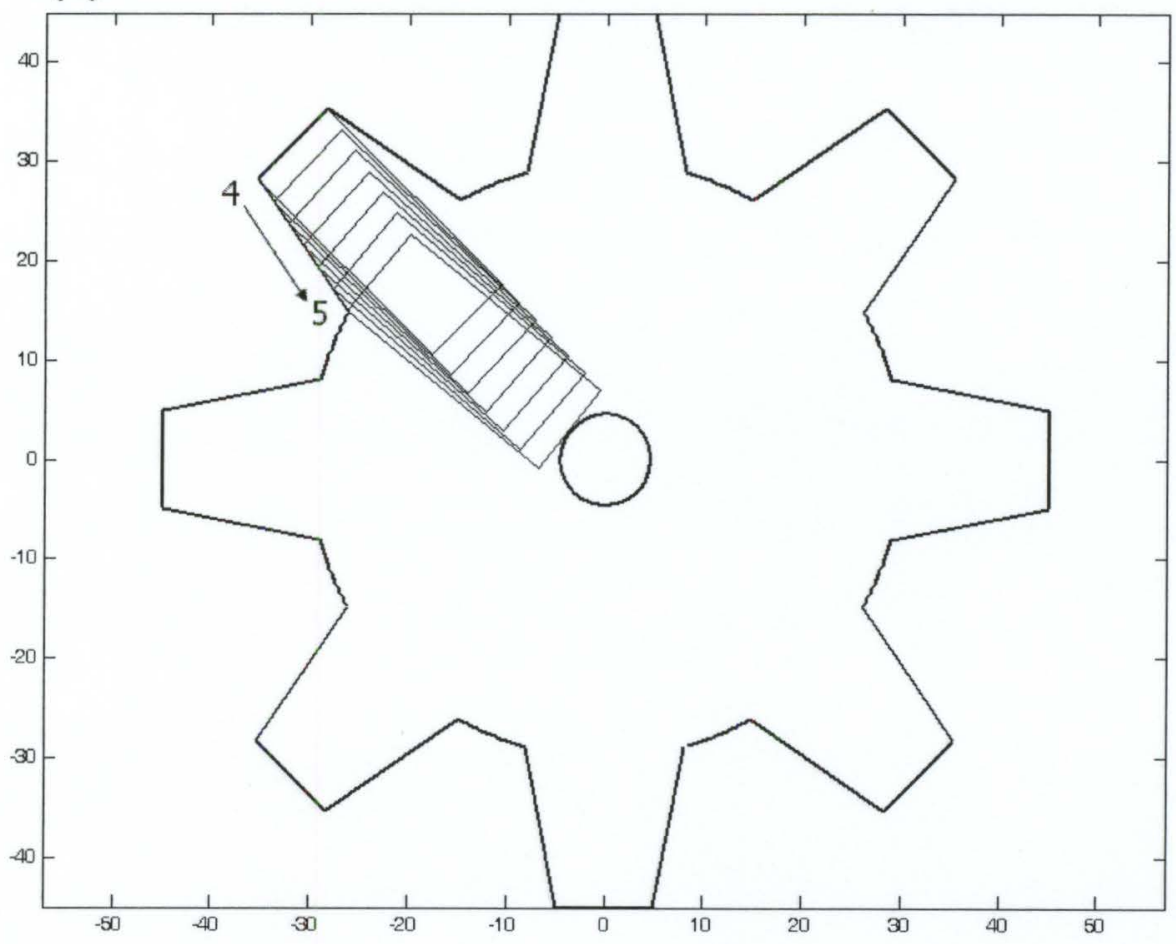

Figure 6.25: Gear Shaped Cavity - Tool Path Segments C \& D 
In Fig. 6.24 and Fig. 6.25, the arrows indicate the motion of the tool with respect to the workpiece and the numbers indicate the start and end points of the segment being traced. The tool path for only one section of the gear is presented as the remainder of the tool path may be determined by symmetry.

The segments of tool path show the leading point of the tools tracing edge being removed from the profile. This occurs when the tool traces the root diameter of the gear. As the leading point leaves the curve being traced, the trailing point remains on it. This requires the trailing point to trace the rest of the curve and thus the entire successive curve.

Machining of this cavity was performed on the AGIE IMPACT 2. The copper tool used during the machining of the cavity was manufactured on a milling machine. To ensure that the front face of the tool was sufficiently flush with the surface of the workpiece, the tool was trued using the System 3R table chuck. Fig. 6.26 shows the tool used in the machining of the gear cavity.

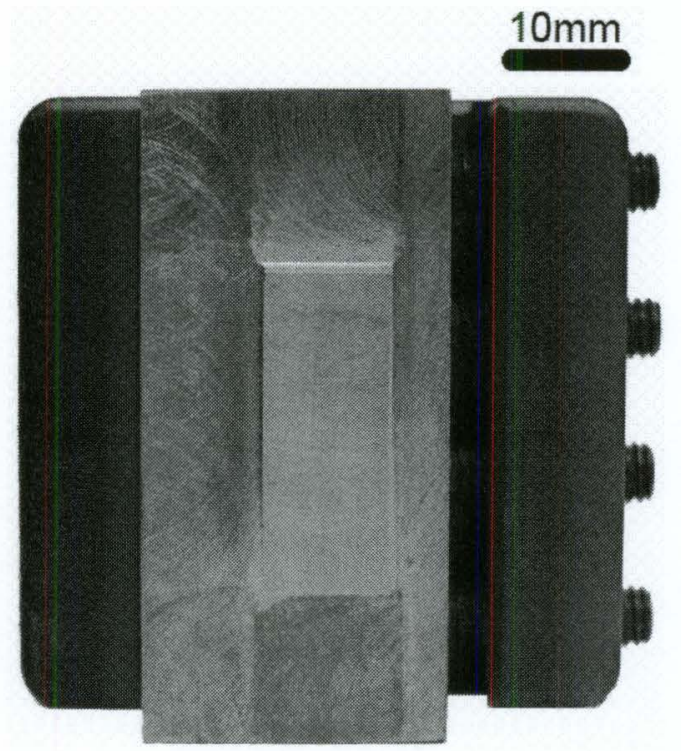

Figure 6.26: Tool used in ED Milling of the Gear Shaped Cavity 
The pulse parameters used during machining are presented in Table 6.4. Machining depth was $1.5 \mathrm{~mm}$ and the total machining time was approximately three hours. Flushing was induced by the relative motion between the tool and the workpiece and was aided by the use of flushing jets. The same setup illustrated in Fig. 6.15 was used during the machining of this cavity.

\begin{tabular}{cc} 
Parameter & Value \\
\hline \hline Current $(\mathrm{A})$ & 21 \\
Voltage $(\mathrm{V})$ & 120 \\
Pulse On-Time $(\mu \mathrm{s})$ & 49 \\
Pulse Off-Time $(\mu \mathrm{s})$ & 49 \\
Polarity & +
\end{tabular}

Table 6.4: Gear Shaped Cavity - Pulse Parameters

Fig. 6.27 presents the cavity machined by ED milling. Like the star cavity, the bottom surface was not flat. This may be attributed to tool wear and overlap encountered during the tool path.

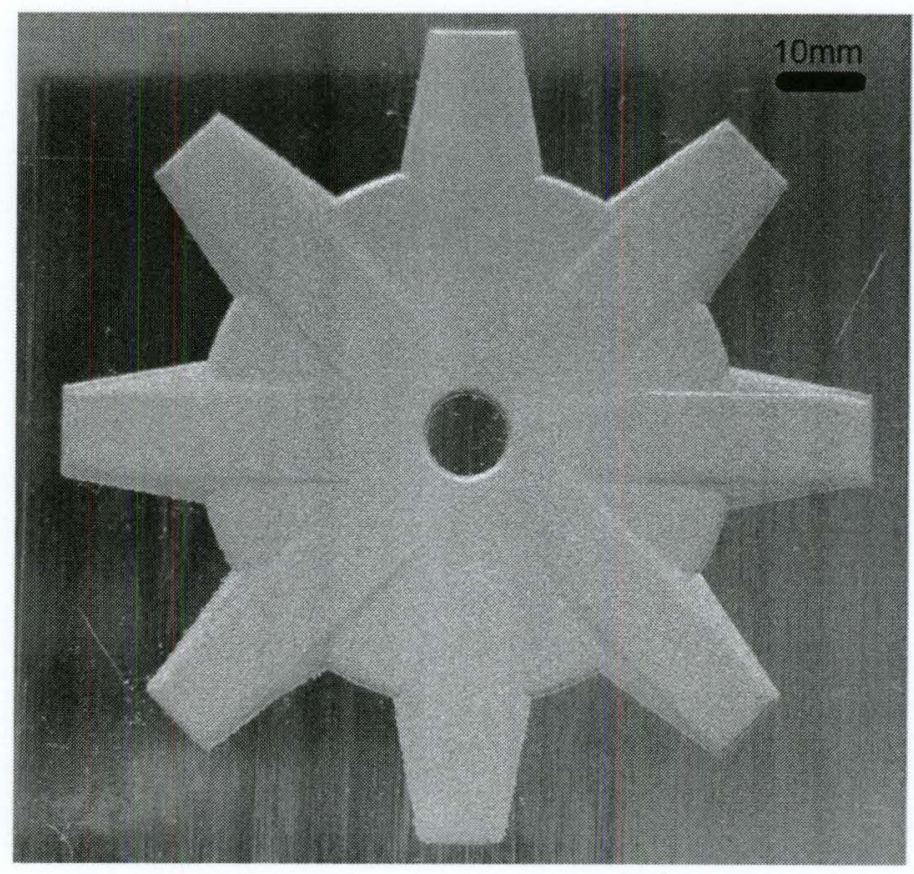

Figure 6.27: Gear Shaped Cavity Machined by ED Milling with Tool Type 2 
Fig. 6.27 shows the successful machining of the gear shaped cavity. Application of the approach presented in this work, as it applies to the gear shaped cavity, resulted in a substantial increase in the frontal area of the tool and machining of an entire layer with a single pass.

Another advantage of this approach lies in the geometry of the gear cavity. The manufacture of a die-sinking tool for this cavity is not easily realized due to sharp corners on the profiles exterior. When manufacturing the die-sinking tool for this cavity, the sharp corners become interior since the cavity and tool are opposed in die-sinking. Since cylindrical cutters are used in the milling process, it is not a feasible option for manufacturing a gear shaped tool. Therefore, another process, such as wire EDM, is required to manufacture the die-sinking tool. The result is an increase in lead time and tooling costs.

The examples provided in this section have shown the benefits of this work. When compared to ED milling with cylindrical/square tools, this method provides a consistent increase in tool size and a reduction in the number of passes required for the complete machining of a layer. Against die-sinking, this method reduces tooling costs and provides ample space for flushing the gap. 


\section{Chapter 7}

\section{Conclusions and Recommendations}

In this section, the algorithms developed for ED milling with oblong tools are summarized. The major benefits of this work are recapitulated, while issues requiring further investigation are addressed.

\subsection{Summary}

In this work, a tool set was developed for the ED milling of $2 \mathrm{D}$ cavities. The set contains three oblong tools which are capable of machining round features and sharp corners. Though it is possible to machine both round and sharp features with die-sinking EDM, the machining of sharp corners is not possible with the cylindrical tools currently used in ED milling. Due to their elongated shape, the tools described in this work have a greater frontal area and therefore, higher power capacity than cylindrical tools. Though die-sinking tools maximize power capacity, they provide little space for flushing debris from the gap. Cylindrical tools and those presented in this work provide ample space for flushing. Another disadvantage to using die-sinking tools is the associated tooling costs. As the complexity of the cavity increases, the cost 
of the die-sinking tool rises. Cylindrical tools are inexpensive and readily available while the tools in this work are cost effective and easy to manufacture.

In addition to the development of a new tool set for ED milling, this work presented algorithms for tool type selection and tool size calculation. According to continuity, concavity and geometric composition the profiles were divided into three groups. Appropriate tool types were associated with each of the profile groups allowing for automated tool selection.

The tools used in this work are defined by width and length. An algorithm for calculating the maximum permissible tool width was presented which compares the tool width defined by features of minimum radius/length with the tool width defined by narrow passages. The maximum permissible tool width was determined to be the minimum of these values. Based on the selected tool width, tool lengths in both concave and convex regions of the profile were calculated. The minimum of these lengths defined the maximum permissible tool length.

Tool path planning strategies were presented for each profile/tool type. The number of passes required to completely machine a single layer of the cavity was determined using an offset approach. Critical points and endpoints of the offset curves indicated locations along the tool path where the tool orientation changed. Changes in tool orientation were determined such that the tool did not intersect the profile boundary. Constraints between the tracing edge of the tool and the profile/profile offset were discussed for both round and square edges. 


\subsection{Conclusions}

Application of the algorithms was presented in the machining of star and gear shaped cavities. For the star shaped cavity, the maximum sized tool was found to be $10.0 \mathrm{~mm}$ in width and $46.6 \mathrm{~mm}$ in length. When compared to an equivalent cylindrical tool, $10 \mathrm{~mm}$ in diameter, a $450 \%$ increase in frontal area was achieved. However, to show the flexibility of this approach, the selected tool size was $10 \mathrm{~mm}$ in width and $25 \mathrm{~mm}$ in length resulting in a $200 \%$ increase in frontal area. The tool path developed for the layer-by-layer machining of the star shaped cavity resulted in the complete machining of a single layer with only one pass. This result is not possible using a cylindrical tool.

For the gear shaped cavity, the maximum tool size was determined as $10 \mathrm{~mm}$ in width and $25 \mathrm{~mm}$ in length. This tool was chosen to machine the cavity and provided a $150 \%$ increase in frontal area over an equivalent square tool having sides $10 \mathrm{~mm}$ in length. Due to the increase in frontal area, the machining of each layer was achieved in only one pass.

The gear shaped cavity contained sharp corners on both its interior and exterior. In terms of tool manufacture, these features make it difficult to machine this cavity by die-sinking. To successfully machine the tool, a process such as wire EDM is required resulting in an increase in tooling costs and lead time.

After machining of both cavities, the profile was clearly defined. However, the bottom surface of the cavities was uneven due to tool wear and overlapping tool paths. This may be corrected in semi-finishing and finishing operations or by appropriate modification of the tool path. 


\subsection{Recommendations}

Implementation of the algorithms presented in a CAD/CAM package should be considered due to the large amount of geometric information stored in such systems. The information available would simplify the calculations and decrease the computation time required. One of the issues with implementation, however, is the computation of the MAT. The MAT must be determined with caution because the algorithms used for computing it are sensitive to deviations in the profile boundary.

Another issue which requires more attention is the unevenness on the bottom surface of the machined cavities. This is a result of two issues. The first, which has been addressed in the literature, is wear. Due to wear, the frontal area of the tool changes shape causing unevenness along the bottom surface of the cavity. This may be remedied by applying one of the many wear compensation techniques to the tool path. A second reason for unevenness along the bottom surface of the cavity is overlapping tool paths. When the tool traces the same region of the cavity more than once in a given layer, that region experiences more discharges and thus, more material removal. Therefore, these regions will likely be deeper than those regions traced only once. Approaching this problem such that the tool is sized according to a non-overlapping tool path may produce a more even surface along the bottom of the cavity.

The determination of pulse parameters based on tool size must also be addressed. To achieve the optimal current density, and therefore the maximum MRR, a set of experiments must be carried out using the tools described in this work. This will establish a relationship between tool area and current density while capturing any geometric effects imposed by the tool and the motion experienced during machining. 
One final issue that should be studied are the limitations of using oblong tools. A list of criteria should be established which identifies when the use of cylindrical tools is more appropriate than the use of the tools presented in this work. In some cases, the increase in frontal area and the complexity of the tool path may not offer substantial benefits over the use of cylindrical tools. Automation of this decision should be implemented to minimize user intervention. 


\section{References}

[1] K. Lee. Principles of $C A D / C A M / C A E$ Systems. Addison Wesley Longman Inc., 1999.

[2] J. Tlusty. Manufacturing Processes and Equipment. Prentice Hall, 2000.

[3] D.T. Pham, S.S. Dimov, S. Bigot, A.Ivanov, and K. Popov. Micro-EDM - recent developments and research issues. Journal of Materials Processing Technology, 149:50-57, 2004.

[4] M. Kunieda, B.Lauwers, K.P. Rajurkar, and B.M. Schumacher. Advancing EDM through fundamental insight into the process. Annals of the CIRP, 54(2):599623, 2005.

[5] AGIE - AGIETRON IMPACT, INNOVATION, EXACT Equipment Manual.

[6] A. Arthur, P.M. Dickens, and R.C. Cobbs. Using rapid prototyping to produe electrical discharge machining electrodes. Rapid Prototyping Journal, 2:4-12, 1996.

[7] Charmille Technologies (2006). EDM applications. http://www.charmilles.com/charmillescom/en/menu_0/application.html., August 30, 2007.

[8] K.H. Ho and S.T. Newman. State of the art electrical discharge machining EDM. International Journal of Machine Tools and Manufacture, 43:1287-1300, 2003.

[9] M. Bayramoglu and A.W. Duffill. CNC EDM of linear and circular contours using plate tools. Journal of Materials Processing Technology, 148:196-203, 2004.

[10] J. Fleischer, J. Schmidt, M Knoll, E. Salim, and F.Leleu. Zündender funke für die mikrotechnik. Mikroproduktion, pages 36-38, 2004.

[11] J.R. Crookall and A.J.R Moncrieff. A theory and elvaluation of tool-electrode shape degeneration in electro-discharge machining. Proceedings of IMechE, 187(6):51-61, 1973. 
[12] J.R. Crookall. A theory of planar electrode-face wear in EDM. Annals of the CIRP, 28(1):125-129, 1979.

[13] N. Mohri, M. Suzuki, M. Furuya, and N. Saito. Electrode wear process in electrical discharge machining. Annals of the CIRP, 45(1):165-168, 1995.

[14] C. Cogun, S. Akasian, and B. Kaftanoglu. An investigation on tool wear in electrical discharge machining (EDM). Canadian Society of Mechanical Engineers, 25(3-4):411-416, 2001.

[15] T. Kaneko and M. Tsuchiya. Three-dimensional numerical controlled contouring by electrical discharge machining with compensation for the deformation of cylindrical tool electrodes. Precision Engineering, 10(3):157-163, 1988.

[16] E. Orhant C. Tricarico, B. Forel. Measuring device and method for determining the length of an electrode. U.S. Patent 6072143, Charmilles Technologies S.A., 2000 .

[17] P. Bleys, J.P. Kruth, and B. Lauwers. Sensing and compensation of tool wear in milling EDM. Journal of Materials Processing Technology, 149:139-146, 2004.

[18] P. Bleys, J.P. Kruth, B. Lauwers, A.Zryd, R. Delpretti, and C. Tricarico. Realtime tool wear compensation in milling EDM. Annals of the CIRP, 51(1):157-160, 2002 .

[19] D. Dauw. On the derivation and application of a real-time tool wear sensor in EDM. Annals of the CIRP, 35(1):111-116, 1986.

[20] Z.Y. Yu, T.Masuzawa, and M. Fujino. Micro-EDM for three-dimensional cavities - development of the uniform wear method. Annals of the CIRP, 47(1):169-172, 1998.

[21] S. Ding and R. Jiang. Tool path generation for 4-axis contour EDM rough machining. International Journal of Machine Tools and Manufacture, 44:14931502, 2004.

[22] W. Zhao, Y. Yang, Z. Wang, and Y. Zhang. A CAD/CAM system for micro-EDmilling of small 3d freeform cavity. Journal of Materials Processing Technology, 149:573-578, 2004.

[23] K.P. Rajurkar and Z.Y. Yu. 3d micro-EDM using CAD/CAM. Annals of the CIRP, 49(1):127-130, 2000.

[24] S. Dimov, D.T. Pham, A. Ivanov, and K. Popov. Tool-path generation system for micro-electro discharge machining milling. Proceedings of IMechE - Journal of Engineering Manufacture, 217:1633-1637, 2003. 
[25] Z.Y. Yu, J. Kozak, and K.P. Rajurkar. Modelling and simulation of micro EDM process. Annals of the CIRP, 52(1):143-146, 2003.

[26] J. Narasimhan, Z. Yu, and K.P. Rajurkar. Tool wear compensation and path generation in micro and macro EDM. Transactions of NAMRI/SME, 32:151$158,2004$.

[27] R. Snoeys, F. Staelens, and W. Dekeyser. Current trends in non-conventional material removal processes. Annals of the CIRP, 35(2):467-480, 1986.

[28] T. Masuzawa and C.J Heuvelman. A self-flushing method with spark-erosion machining. Annals of the CIRP, 32(1):109-111, 1983.

[29] W. Kónig, L. Jórres, and U. Behmer. The kinematics of planetary erosion as a basis for electro-discharge polishing and process optimisation strategies. Advanced Manufacturing Processes, 2(1-2):23-35, 1987.

[30] F. Staelens and J.P. Kruth. A computer aided machining strategy for planetary EDM. Annals of the CIRP, 38(1):187-190, 1989.

[31] J.A. Sanchez, L.N. Lopez de Lacalle, A. Lamikiz, and U. Bravo. Dimensional accuracy optimisation of multi-stage planetary EDM. International Journal of Machine Tools and Manufacture, 42:1643-1648, 2002.

[32] G. Wang and Y. Shan. Compensation of electrode orbiting in electrical discharge machining based on non-unifrom offsetting. International Journal of Machine Tools and Manufacture, 45:1628-1634, 2005.

[33] M. Bayramoglu and A.W. Duffill. Manufacturing linear and circular contours using CNC EDM and frame type tools. International Journal of Machine Tools and Manufacture, 35(8):1125-1136, 1995.

[34] K. Saito, H. Konno, and M. Sato. Development of numerical contouring control electrical discharge machining (NCC-EDM). Annals of the CIRP, 35(1):117-120, 1986 .

[35] Y. Ziada and P. Koshy. Rotating curvilinear tools for edm of polygonal shapes with sharp corners. Annals of the CIRP, 56(1):221-224, 2007.

[36] J. Valentinčič, D. Kušer, S. Smrkolj, and M. Junkar. Selection of optimal EDM parameters for the given machining surface. In 8th International conference on management and innovation technologies MIT, pages 217-225, 2005.

[37] M.L. Jeswani. Effect of machining area and pulse parameters on EDM performance. Indian Journal of Technology, 18:354-356, 1980. 
[38] H. Blum. A transformation for extracting new descriptors of shape. Technical report, MIT Press, 1967.

[39] M. Ramanathan and B. Gurumoorthy. Constructing medial axis transfrom of planar domains with curved boundaries. Computer-Aided Design, 35:619-632, 2002.

[40] H. Blum and R. Nagel. Shape description using weighted symmetric axis features. Pattern Recognition, 10:167-180, 1978.

[41] E. Remy and E. Thiel. Exact medial axis with euclidian distance. Image and Vision Computing, 23:167-175, 2005.

[42] M. Held. VRONI: An engineering approach to the reliable and efficient computation of voroni diagrams and line segments. Computational Geometry, 18:95-123, 2001.

[43] M. Held. Private communication, February 20, 2007.

[44] A. Hatna, R. J. Grieve, and P. Broomhead. Automatic CNC milling of pockets: geometric and technological issues. Computer Integrated Manufacturing Systems, 11(4):309-330, 1998.

[45] K. Tang, M. Wang, L. Chen, S. Chou, T. Woo, and R. Janardane. Computing planar swet polygons under translation. Computer-Aided Design, 29(12):825-836, 1997.

[46] K. Sambandan, K. Kedem, and K. K. Wang. Generalized planar sweeping of polygons. Journal of Manufacturing Systems, 11(4):246-257, 1992.

[47] Z. Ling and T. R. Chase. Generating the swept area of a body undergoing planar motion. Transactions of the ASME, 118:186-192, 1996. 